NOVEMBER 2017

\title{
The Growing Value of Digital Health
}

Evidence and Impact on Human Health and the Healthcare System

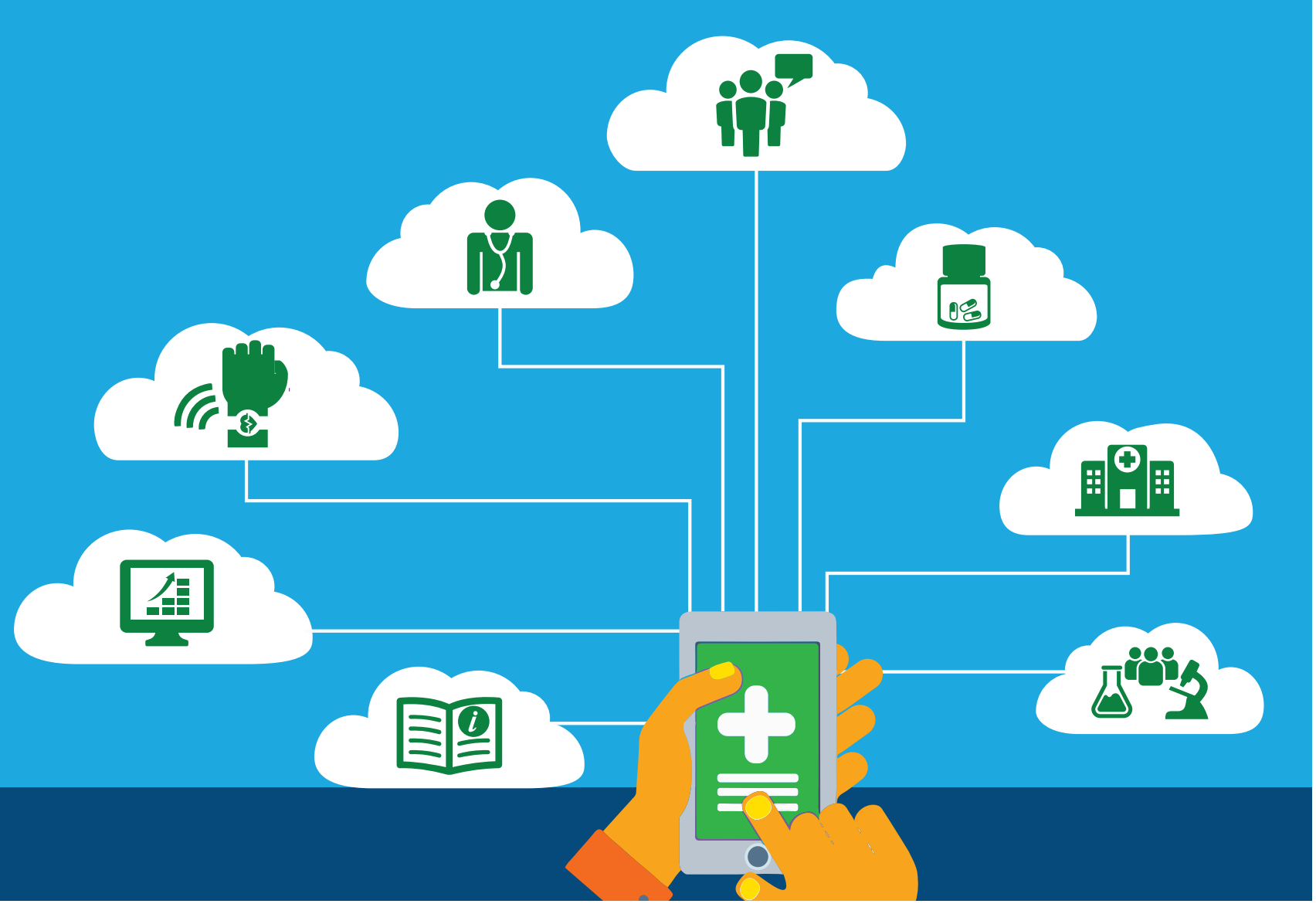




\section{Introduction}

The proliferation of Digital Health tools, including mobile health apps and wearable sensors, holds great promise for improving human health. As with other new health technologies, evidence of their effectiveness is a fundamental requirement of the health system and a limiting first step to adoption into clinical practice. Although analyses of the Digital Health landscape published by the IMS Institute for Healthcare Informatics in 2013 and 2015 found evidence still to be scarce and the value of Digital Health difficult to measure, this has now changed and the benefits to patients are becoming clearer. Efforts to incorporate these tools into practice are underway.

This study of Digital Health examines trends in three areas - innovation, evidence and adoption - to examine whether these new tools are positioned to have a fundamental impact on patient care. As digital tools focused on the detection, prevention, and management of specific diseases proliferate, this report explores the growing body of evidence that demonstrates their impact on human health and extrapolates the potential cost savings to the U.S. healthcare system. Further, as mobile apps are increasingly partnered with wearables and other sensors that objectively monitor measures of health, their ability to track patient experience, health risk and outcomes in real time as "digital biomarkers" are explored. Use of mobile technologies by various stakeholders, including pharmaceutical companies in clinical trials, health systems in disease management and payers in wellness programs, are described, as well as the barriers and accelerators to their further use. report of Christine Lemke, President, Evidation Health; Marc Sebes, Vice President of Product Management, Validic; Dr. Michael Hodgkins, Vice President and Chief Medical Information Officer, American Medical Association; David Vinson, Founding Director and Vice Chairman of Xcertia and Founder of DHX group; as well as Michael Krupnick, Hilary Armstrong, John Doyle, Nelia Padilla, Greg Plante and dozens of others at IQVIA are gratefully acknowledged.

\section{Find Out More}

If you wish to receive future reports from the IQVIA Institute for Human Data Science or join our mailing list, visit iqviainstitute.org

MURRAY AITKEN

Executive Director

IQVIA Institute for Human Data Science

This study was produced independently by the IQVIA Institute as a public service, without industry or government funding. The contributions to this

C2017 IQVIA and its affiliates. All reproduction rights, quotations, broadcasting, publications reserved. No part of this publication may be reproduced or transmitted in any form or by any means, electronic or mechanical, including photocopy, recording, or any information storage and retrieval system, without express written consent of IQVIA and the IQVIA Institute. 


\section{Table of contents}

$\begin{array}{lr}\text { Executive summary } & 1\end{array}$

Proliferation of Digital Health tools $\quad 3$

Mobile apps $\quad 4$

$\begin{array}{ll}\text { Wearable biosensors and other connected devices } & 7\end{array}$

$\begin{array}{lr}\text { Innovative uses of sensors } & 12\end{array}$

$\begin{array}{ll}\text { Smart devices encouraging adherence } & 12\end{array}$

$\begin{array}{ll}\text { Digital diagnostics guiding care } & 14\end{array}$

$\begin{array}{lr}\text { Transforming clinical trials } & 18\end{array}$

$\begin{array}{lr}\text { Delivering value to patients and the health system } & 22\end{array}$

$\begin{array}{ll}\text { Value along the patient journey } & 22\end{array}$

$\begin{array}{lr}\text { A growing body of evidence } & 28\end{array}$

$\begin{array}{ll}\text { Top Apps } & 34\end{array}$

Overall value to the healthcare system $\quad 36$

$\begin{array}{ll}\text { Investments in evidence } & 40\end{array}$

$\begin{array}{ll}\text { Current trials using Digital Health } & 40\end{array}$

Sponsorship of Digital Health trials $\quad 43$

Accelerating use in medicine $\quad 46$

The path to health system adoption 46

$\begin{array}{ll}\text { Accelerators and barriers } & 47\end{array}$

$\begin{array}{lr}\text { Appendix } & 57\end{array}$

$\begin{array}{lc}\text { References } & 63\end{array}$

$\begin{array}{ll}\text { About the authors } & 71\end{array}$

$\begin{array}{lr}\text { About the IQVIA Institute } & 72\end{array}$ 


\section{Executive summary}

Over 318,000 health apps and 340 consumer wearable devices are now available worldwide. The value of these Digital Health tools to human health and the healthcare system is still evolving. There has been continued adoption by various stakeholders, exploration of innovative ways to apply these tools to health and growing evidence of their impact on human health outcomes. Since our study in 2015, over 153,000 new Digital Health apps were introduced to the Apple Store and Google Play, nearly doubling the number available, with more than 200 health apps being added each day. Apps are being released worldwide, though few are available beyond the major world languages, thus limiting their potential impact on global populations. General wellness apps still account for the majority of health apps available to consumers, but those focused on health condition management - often associated with patient care - are growing and now account for $40 \%$ of all apps.

Over 55\% of the most downloaded health apps now use sensor data, with significant adoption of consumer wearables like Fitbit and Jawbone for wellness management being a key driver of this phenomenon. The next wave of innovation being applied to sensor technologies - including smartphone sensors, wearables and vital-sign-specific sensors - brings with it significant possibility to improve health by supporting condition management. Detecting various health parameters and vital signs with accuracy, these connected sensors stand to replace bulky clinical devices, rival clinical wearables, and may enable population-based screening and monitoring.

Digital sensors linked to apps are bringing innovation in three key areas: the creation of smart devices, digital diagnostics, and new human-centered clinical trial designs. Digital-enabled "smart" devices such as asthma smart inhalers, connected pens for diabetes and smart blister packs are being developed to encourage patient adherence. These have shown improved therapeutic outcomes and smart inhalers are moving to become the new standard of care in the asthma space. New value will also be brought to healthcare by algorithms built on top of activity monitors to create "digital biomarkers" of health. By tracking parameters beyond just sleep and steps that correlate to disease severity, they will contribute to precision medicine, enabling stratification of patients by symptoms identified by sensors, in addition to traditional biomarkers. Sensors further offer to improve clinical trial designs by enabling the collection of patient experience data in the "real world," even within the bounds of clinical trials. They also offer a route to speed patient recruitment and retention and improve trial efficiency.

Although the range of health apps available present an overwhelming amount of options for consumers to choose from without guidance from their healthcare provider, there are now established leaders among apps for consumers to use. Just 41 apps with over 10 million downloads each account for nearly half of all app downloads while over $85 \%$ of all health apps have fewer than 5,000 installs. There is now also at least one high-quality app for each for each step through the patient journey. The importance of Digital Health to healthcare is defined by such apps that are the best in each use category, and the value they deliver, while the plethora of lesser apps have little impact. These leading apps may have high patient ratings, frequent updates, connectivity to sensors, inclusion in healthcare institutions' app formularies, endorsements, and promising clinical evidence.

The overall body of clinical evidence on app efficacy has grown substantially, including randomized controlled trials (RCTs) and meta-analysis studies, enabling the identification of a list of apps with strong clinical evidence. Particularly strong evidence now exists for use in diabetes, depression and anxiety, making these 
categories strong candidates for consideration by clinical guidelines writers. An additional 24 categories have one or more RCTs with positive results making associated apps strong candidates for adoption by healthcare stakeholders (e.g., provider organizations and payers).

The use of Digital Health apps in just five patient populations where they have proven reductions in acute care utilization (diabetes prevention, diabetes, asthma, cardiac rehabilitation and pulmonary rehabilitation) could save the U.S. healthcare system an estimated $\$ 7$ billion per year. This represents about 1.4\% of total costs in these patient populations. If this level of savings could be achieved across all disease areas, annual cost savings of $\$ 46$ billion could be achieved.

These estimates extrapolate just from existing evidence, but continued investment in evidence generation continues across stakeholders. Over 860 clinical trials worldwide now incorporate Digital Health tools, including 540 in the U.S., with two thirds of these focused on apps and text message interventions to smartphones. The vast majority (82\%) of these trials are sponsored by universities, hospitals, health systems and other patient care institutions, demonstrating the increased efforts to fit Digital Health into clinical practice. The academic and institutional sponsors with the most research in this area are currently Duke University, the University of California San Francisco, and the University of Pennsylvania.

Despite progress to date, a number of barriers still exist to widespread adoption by patient care institutions, and only an intermediate level of adoption has yet occurred. Only $26 \%$ of clinicians recommend patient engagement technologies and $13 \%$ use remote patient monitoring technologies according to an American Medical Association (AMA) survey. Barriers to further use by physicians surround app selection, concerns around privacy, security and malpractice liability, financial incentives, and workflow integration. Few condition management apps - which offer the greatest potential impact on human health care and healthcare costs - have reached the level of workflow integration necessary to gain widespread physician adoption.

Before healthcare can be more fully supported by apps and sensors, a new fit-for-purpose infrastructure must evolve to support their delivery and incorporation into the standard health toolkit, similar to the type of ecosystem that exists to disseminate safe therapeutics. A variety of industry and policy initiatives have now emerged to address these barriers and accelerate the ongoing adoption of Digital Health tools by care facilities. Critically, curation platforms are facilitating the creation of Digital Therapeutics formularies; privacy and security guidelines are being published; providers are now incentivized for use of Digital Health through value-based payments, and data integration vendors have stepped in to allow healthcare stakeholders more easily make use of Digital Health data and tie it to their electronic health records (EHRs). Investments by healthcare and provider organizations in Digital Health continue to mature, with an estimated $20 \%$ of large health systems shifting from pilot programs to more full-scale rollouts. Within the next five years, this progression is likely to be true for most healthcare companies and within ten years the use of Digital Health is likely to be mainstream for most organizations supporting human health. 


\section{Proliferation of Digital Health tools}

- Over 318,000 health apps and over 340 consumer wearable devices are now available worldwide, with over 200 health apps being added each day.

- General wellness apps still account for the majority of health apps, but the number of apps focused on health condition management - those often associated with patient care - are increasing faster and now account for $40 \%$ of all apps.

- Of the top downloaded Android health apps, over $55 \%$ make use of data collected from sensors including activity- and sleep-monitoring wearables.
- The next wave of innovation among wearable sensors focuses on detecting various health parameters and vital signs with accuracy, which may enable population-based screening and monitoring and replace bulky clinical devices.

While "Digital Health" is defined in varying ways, the term is used throughout this report as meaning the use of connected mobile devices - including, but not limited to, mobile phones, tablets, consumer wearables, connected biosensors, and in-home virtual assistants - to improve health (see Exhibit 1). ${ }^{1,2}$ The value of these tools typically derive from abilities to communicate information through the internet, web or text messaging, to provide continuous monitoring of human health metrics or display health data more clearly.

Exhibit 1: Digital Health Tools
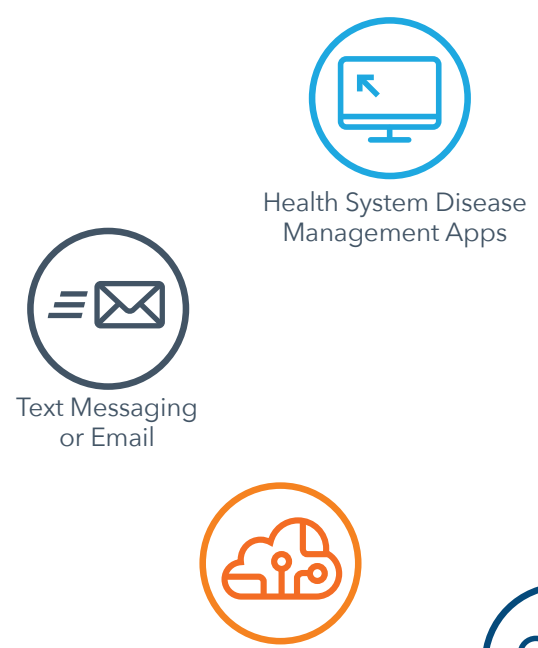

Web-based Interactive Programs

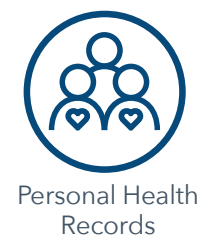

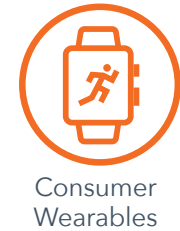

Wearables

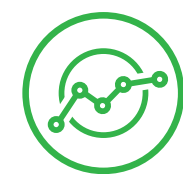

Connected Biometric Sensors

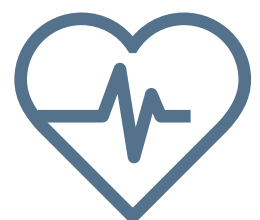

Digital Health

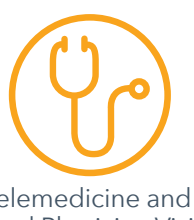
Virtual Physician Visits

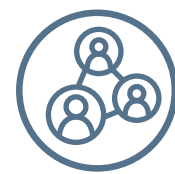

In-Home Connected Virtual Assistants
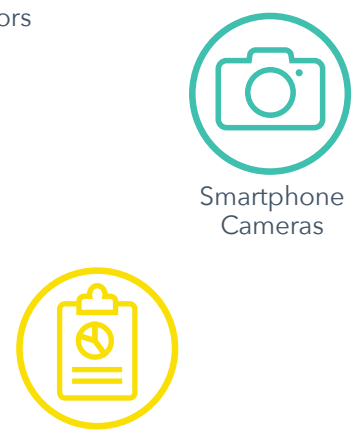

Clinical Trial Patient Information Collection Tools 
Exhibit 2: Number of Digital Health Apps 2013, 2015 and 2017

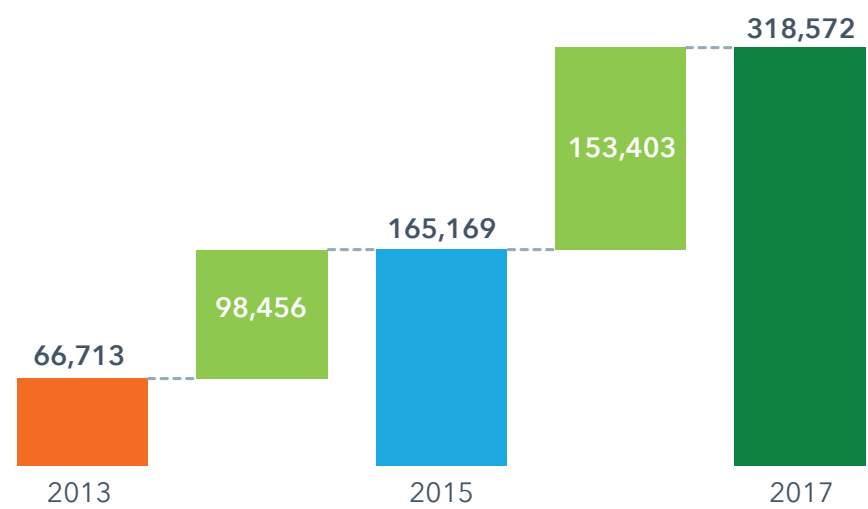

Sources: 42 Matters, Jul 2017; Mevvy, Jun 2015; IQVIA AppScript Database, Jul 2015; IQVIA Institute, Jul 2017

Note: Includes digital health apps publically available to consumers via the Apple Store and Google Play

\section{MOBILE APPS}

The adoption and use of smartphones and other mobile devices is widespread, with $95 \%$ of all Americans (and $80 \%$ of seniors) having cellphones that enable textmessaging and $77 \%$ having smartphones that enable mobile applications - both of which can be used to encourage health behaviors. ${ }^{3}$ Capitalizing on the opportunity this digital audience presents and the growing consumer interest in wellness, innovators continue to fill the market with mobile health apps.

There are now over 318,000 health apps worldwide nearly double the number of apps found in our 2015 analysis and nearly five times those available in 2013. Since our study in 2015, over 153,000 new Digital Health apps were introduced to the Apple Store and Google Play, adding more than 200 new health apps per day on average (see Exhibit 2).

Although $73 \%$ of the over 318,000 health apps are available in English, mobile health apps are increasingly moving beyond English-speaking audiences and are available across the globe in multiple languages. Most countries now have 210,000-250,000 health apps
"The trend line is up and to the right on the percentage of every population who's starting to track [their health digitally] because it's becoming easier and easier to do so. You can look at just smartphone adoption in the Medicare crowd.... It's a common, popular theme to say, 'Oh this isn't going to work for the population who's the most in need of these things, ' but it's just not true. The numbers are still smaller than for 30 year olds who use it but it's not as small as you might think."

Christine Lemke, President, Evidation Health

available in their Google Play and Apple app stores. While this opens up opportunities for Digital Health to support the health and wellness efforts of global populations, over half of apps are still available solely in English (56\%) and most languages are supported by only very few apps (see Exhibit 3), significantly reducing the potential health impact for local populations.

Health apps also appear to be getting better over time, based on user experience, with $55 \%$ of apps in 
Exhibit 3: Digital Health Apps Available in Each Language

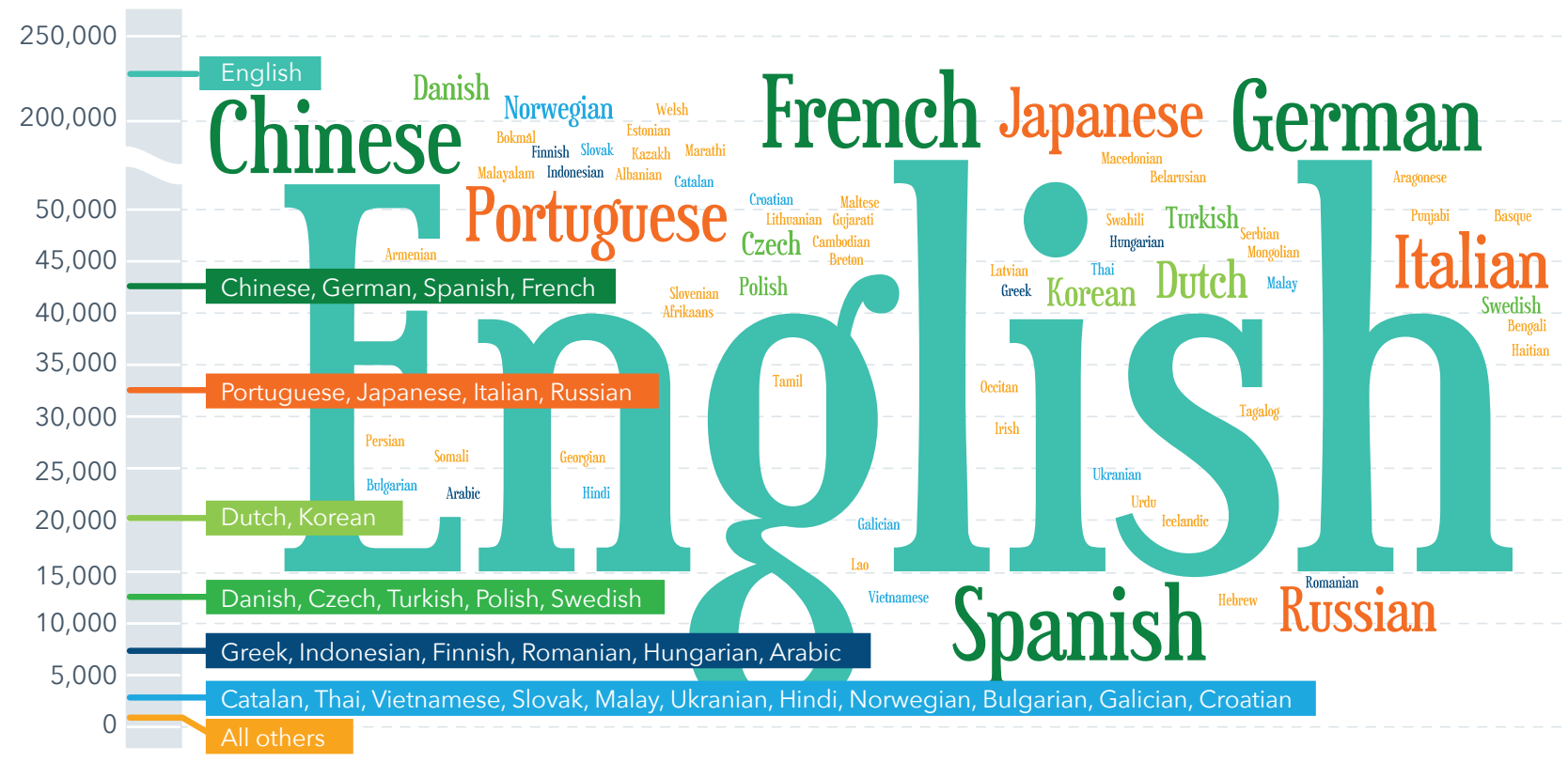

Sources: 42 Matters, Jul 2017; IQVIA AppScript Database, Jul 2017; IQVIA Institute, Sep 2017

Note: Relative word size tied to number of apps available in each language. Languages with fewer than 7,000 apps are depicted as size equivalent to those with 7,000 for visibility. Languages grouped into ranges are placed th the grouping midpoint on the legend.

Exhibit 4: Average Customer Ratings of Health Apps Released Then and Now

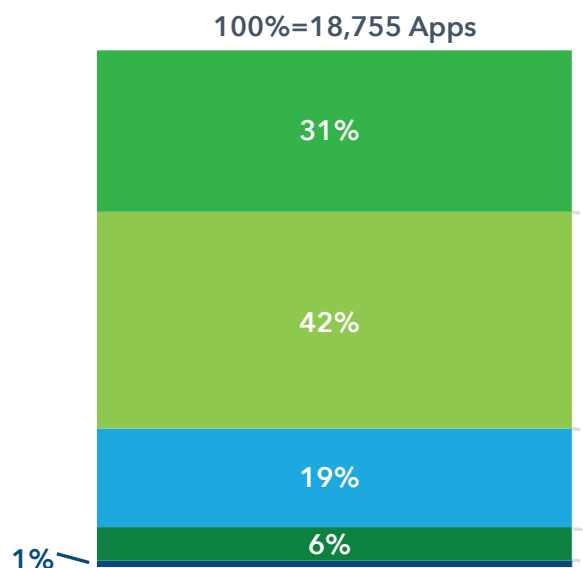

Before Aug 2015

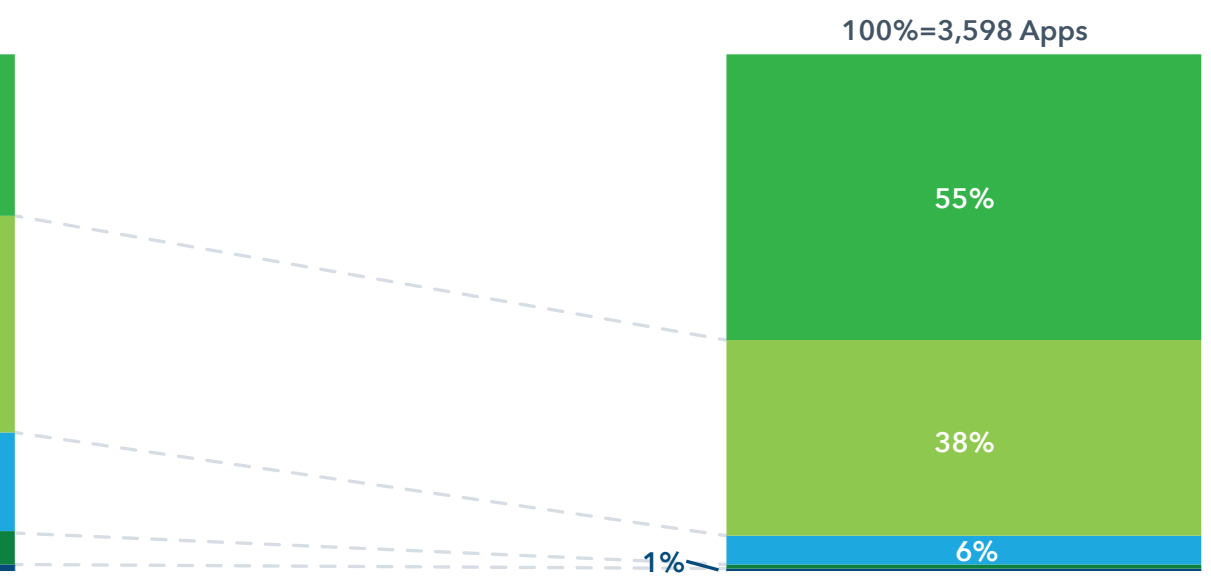

Past 2 Years
$>0-1$
$>1-2$
$>2-3$
$>3-4$
$>4-5$

Sources: 42 Matters, Jul 2017; IQVIA AppScript Database, Jul 2017; IQVIA Institute, Sep 2017

Note: Cohorts defined by app release date. 
the AppScript App Database (see Methodology) that launched in the past two years having ratings above four stars, compared to $31 \%$ of those launched before 2015 (see Exhibit 4). Since there are now good, marketleading apps in many health categories, developers launching apps and getting low star ratings initially may either pull apps more rapidly from the store or invest more continuously in updates based on users' feedback, thus increasing the value of available apps to the consumer. ${ }^{4,5}$ App stores have also begun removing low quality apps, including outdated, abandoned apps, as well as those that no longer meet current guidelines or don't function as intended. 6,7,8

In the current study, apps present in the AppScript App Database (representative of the most widely used Digital Health apps by consumers) were analyzed by use category to understand the use of current landscape of Digital Health apps. Across the patient journey, Digital Health apps can be divided into two main categories: those focused on "wellness management," which facilitate tracking and modification of fitness behaviors, lifestyle and stress and diet, and those which specifically focus on "health condition management" which supply information on diseases and conditions, enable access to care and enable treatment protocols such as medication reminders. Since 2015, consumer Digital Health apps targeting wellness management have dropped as a proportion of total apps from $73 \%$ to $60 \%$, with a corresponding $48 \%$ increase in the share of apps focused on health condition management (see Exhibit 5). Condition management apps now account for $40 \%$ of all Digital Health apps, with notably those focused on specific diseases having grown from $10 \%$ to $16 \%$ of all apps, and those which provide medication reminders having grown from $7 \%$ to $11 \%$.

For apps that provide disease-specific support and management, the top five therapy areas they focus on are all chronic conditions: mental health conditions, diabetes, heart and circulatory conditions, nervous system disorders and musculoskeletal conditions (see

Exhibit 5: Digital Health Apps by Category 2017

Wellness Management

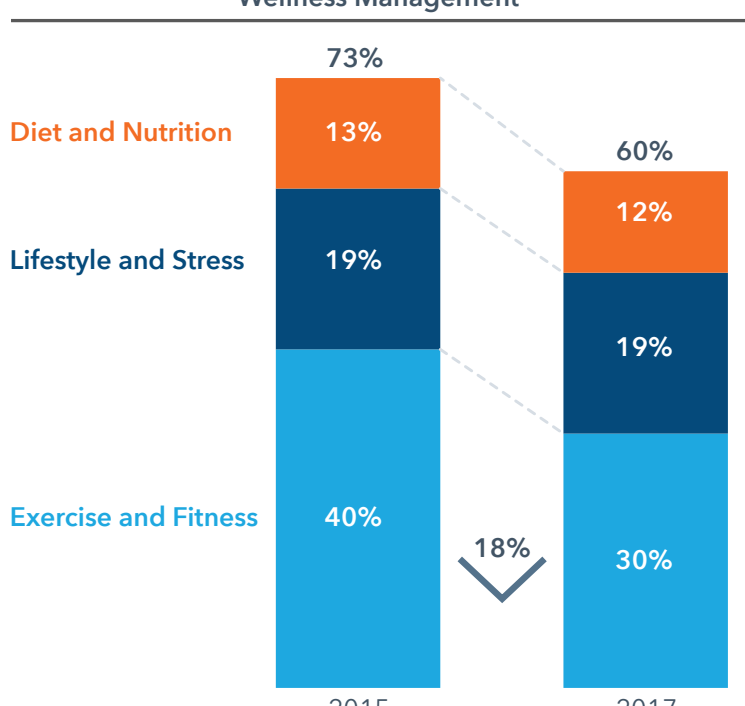

2015
2017
Health Condition Management

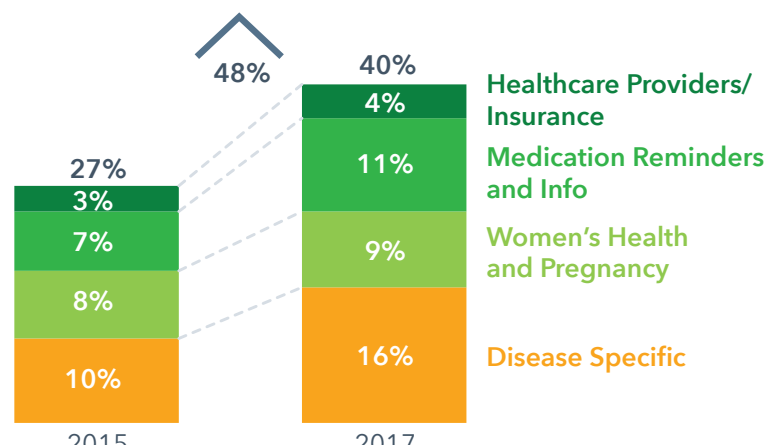

2017

Sources: 42 Matters, Jul 2017; IQVIA AppScript Database, Jul 2017; IQVIA Institute, Jul 2017

Note: Chart displays share of categorizations. Growth normalized for sample. Numbers may not sum due to rounding; 2017 data includes 11,216 unique apps with 11,249 categorizations. 2015 data includes 24,012 apps with 24,088 categorizations. View removes uncategorized apps from 2015 published numbers. 
Exhibit 6). Mental health remains the largest focus for disease-specific mobile apps, with a full third of these dedicated to autism and "augmentative and alternative communication" (AAC). The most commonly addressed mental health conditions are otherwise depression, anxiety and attention deficit hyperactivity disorder.

The range of health apps available present an overwhelming amount of options for consumers to choose from without guidance from their healthcare provider. In the absence of this guidance, consumers will often select the top app in terms of popularity or downloads (a.k.a. installs), such that very few apps account for the majority of downloads. On the Google
Play store, 41 apps with over 10 million downloads each account for nearly half of all downloads (see Exhibit 7). Over $85 \%$ of all health apps have fewer than 5,000 downloads.

\section{WEARABLE BIOSENSORS AND OTHER CONNECTED DEVICES}

Many of these 41 most popular apps also connect to sensors, no longer only relying purely on patient input data. Since the release of Fitbit to the market in 2007, a growing number of individuals have adopted wrist-worn wearables like fitness trackers and smartwatches to help track their activity levels and provide real-time feedback

Exhibit 6: Disease-Specific Apps by Therapy Area

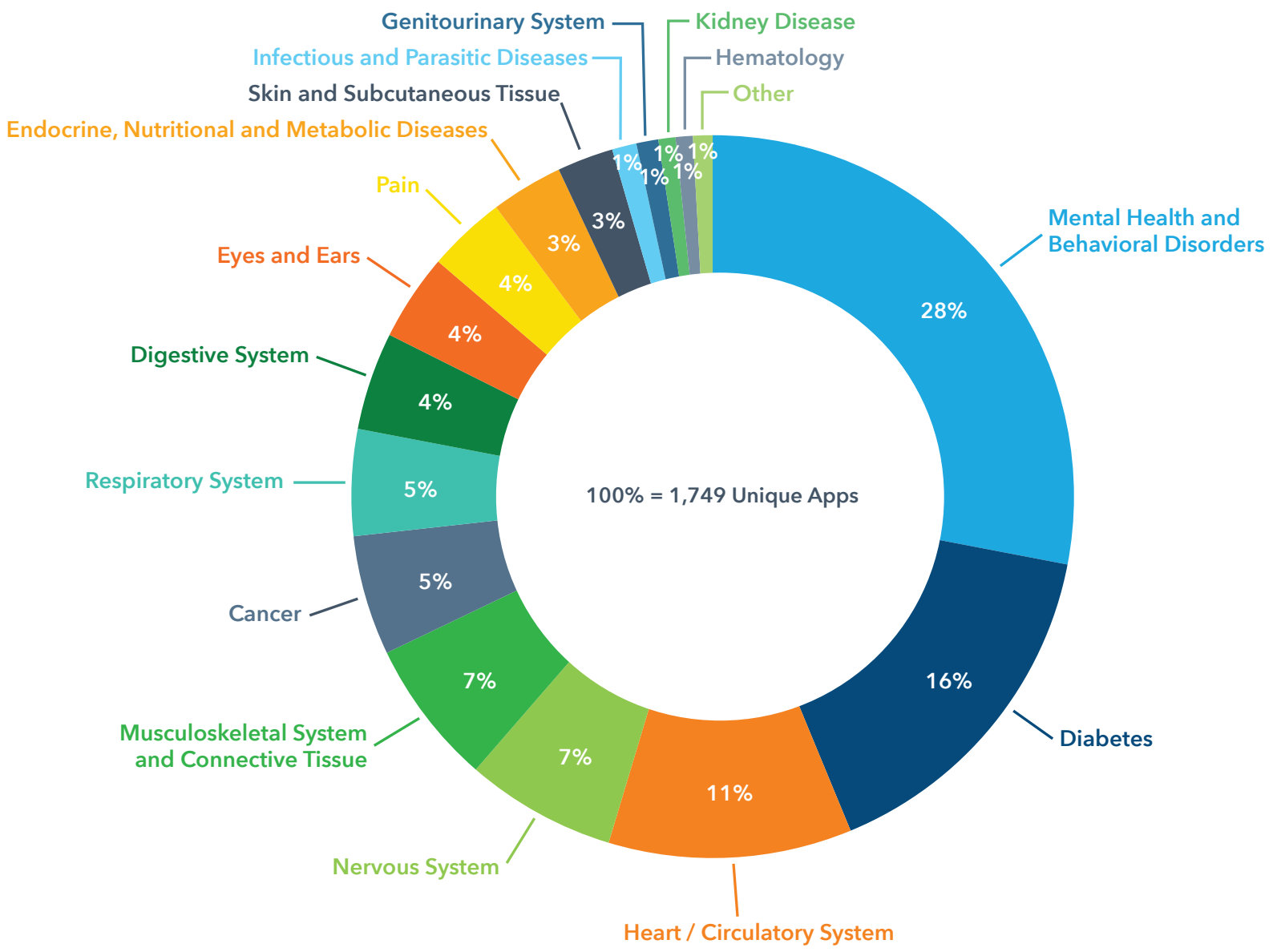




\section{Exhibit 7: Digital Health App Downloads}

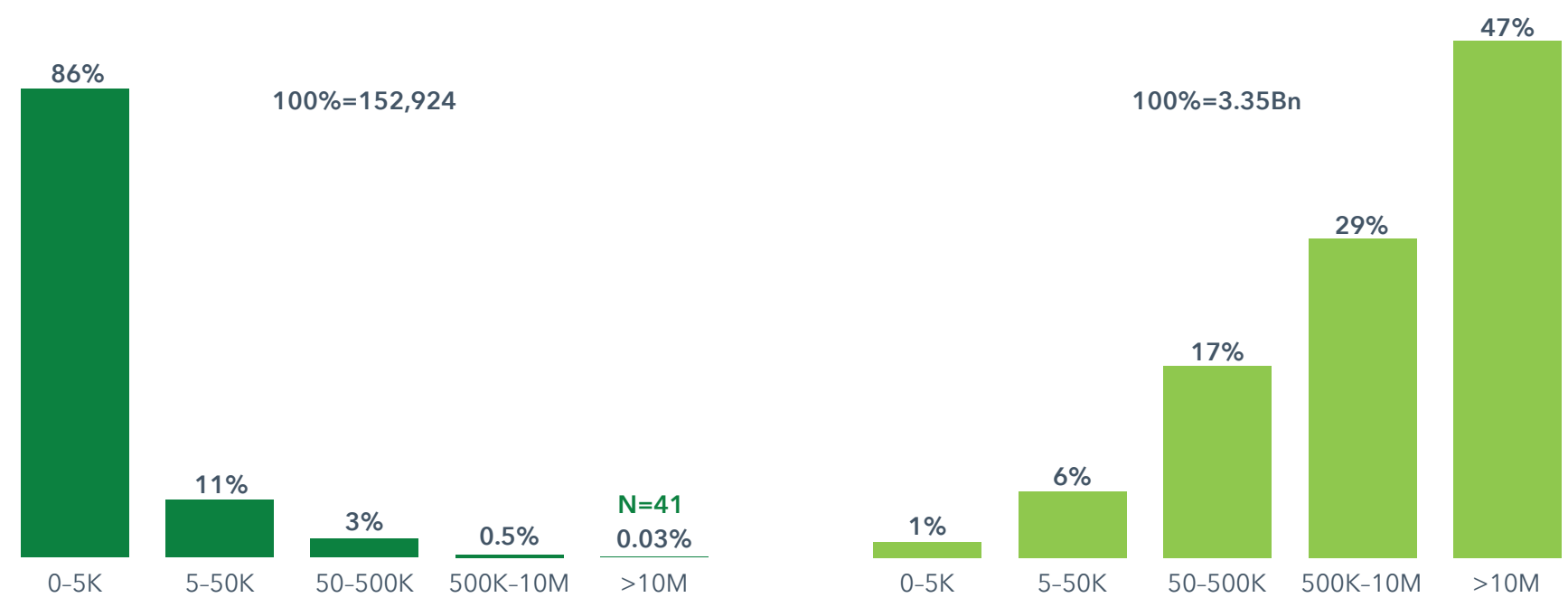

Percent of Total Apps by Install Band

Percent of Total Installs

Sources: 42 Matters, Sep 2017; IQVIA AppScript Database, Sep 2017; IQVIA Institute, Sep 2017 Note: Includes Android apps only. iOS data not available.

to aid in motivation. Among the top downloaded health apps, 34 are exclusively health-focused, and include 22 fitness and general wellness apps, five tracking track food/diet or water intake, three tracking female fertility cycles, two tracking heart rate, one tracking sleep and one prescription filling app. Over 55\% (20/34) of these top health apps make use of sensor data.

Biosensors, as an overall category of devices, collect information on a variety of health parameters and vital signs by reading or measuring energies from a person e.g., pressure, temperature, light, etc. - and transmit that data via electric signals to be interpreted. Among these, activity monitors measure consumer motion patterns (e.g., movement, rotation and position) and translate them into measures of routine activity like sleep, steps and exercise, among others. Although activity monitors have been among the most popular, there are now a vast range of connected biosensors that transmit health information wirelessly to mobile apps (see Exhibit 8). Users can interact with biosensors briefly, such as a glucometer, or wear them for continuous data collection.
Based on analysis of the AppScript Device database, 344 consumer wearable biosensors are now available worldwide, with fitness trackers and smartwatches accounting for $47 \%$ and $13 \%$ of these devices, respectively. While activity monitors primarily track various aspects of patient motion, parameter-specific biosensors, such as those detecting blood glucose levels, blood pressure, heart rhythm, oxygen levels, etc., that purport to diagnose disease, must gain FDA approval. Some of these devices record measurements with high sensitivity and specificity like their clinical device counterparts and therefore offer to transform the use of Digital Health from a consumer novelty to an extension of patient care. A subset also monitor health parameters continuously and passively (without patient effort) offering to simplify the use of digital technologies for older adults managing chronic conditions.

Compared with traditional medical monitoring through bulky equipment installed at hospital facilities or deployed through home-health visits, which is costly to deploy, these new devices hope to offer a route to 


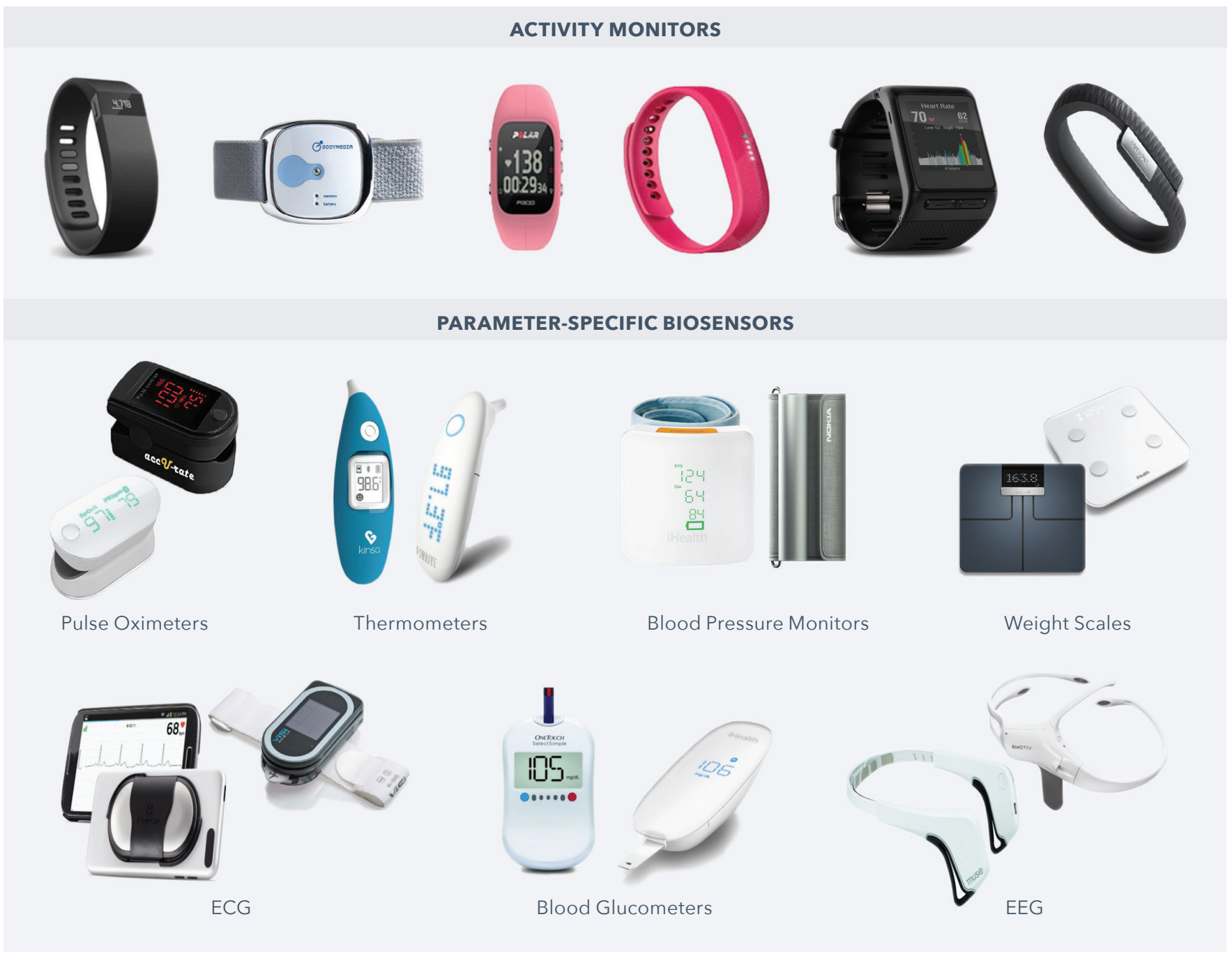

Sources: IQVIA Institute, Sep 2017

Note: Activity monitors include fitness trackers, smartwatches, sleep trackers and actigraphy devices.

more widespread health monitoring outside the walls of health facilities. For instance, Samsung is attempting to measure heart-rate variability, blood pressure, oxygen saturation (Sp02), body fat and other metrics of health and disease on a wearable skin patch (S-patch) with five different sensors. The device would perform bioelectrical impedance analysis (BIA) for body fat detection, photoplethysmography (PPG) for heart rate and respiration, electrocardiogram (ECG) for heart rate and heart rhythm, measure skin temperature (SKT) for overall body temperature, and use galvanic skin response (GSR) and electrodermal activity (EDA) to track stress levels and emotional states. 9,10 The innovation being applied to sensor technologies may see these rival clinical grade wearables in the future in terms of measurement quality of and the value they provide, and could spur further growth in the wearables market. The various measurements tracked by currently marketed biosensors can be seen in Exhibit 9. 
Some mobile apps also use sensor components of smartphones to provide health value. The phone camera can capture the action of a patient taking their medicines (e.g., AiCure) to confirm adherence, while the phone's gyroscope, magnetometer and accelerometer can be used to track activity similarly to an activity tracker. A subset of apps claim to measure vital signs and other key health parameters using a smartphone's camera and light - for example, to track a patient's pulse - or aim to prove value in disease diagnosis. For instance, the Cardiio Rhythm app has compared its ability to detect atrial fibrillation in head-to-head studies versus the gold standard in detection as well as an FDA approved external consumer wearable, AliveCor's EKG device.11,12,13 In these cases the apps would require the approval of the FDA, which reviews apps intended to be used as an accessory to a regulated medical device, or which transform a mobile platform into a regulated medical device. ${ }^{14}$ While app detection without the need for additional hardware beyond the smartphone would be significant to enable population-based screening, many apps have claimed to measure vital signs and other key health parameters without obtaining FDA clearance or have made unsubstantiated claims. In these cases, the Federal Trade Commission (FTC) and state Attorney General offices have pursued legal cases against them.

Providing that mobile apps and their connected sensors do what they say they do, the potential value of sensor data to healthcare is significant - especially in the remote monitoring of human health. To follow exacerbations in patients with chronic conditions, physicians have been dependent on patient reported changes in their vital signs like blood pressure and glucose levels, or oftentimes lost track of patients entirely once they left the office. Wearable biosensors, alternately, can track health data in near real-time to provide information to physicians that is more objective and potentially more timely than patient reported data, with added ability to provide alerts to caregivers when vital sign values fall outside set health parameters; enabling individuals to avoid negative health outcomes.
"One of the most commonly downloaded apps in the Apple Store...said it could measure your blood pressure if you held it up to your chest. I think you had to put your finger on the camera. Most of the time it told patients who were hypertensive that they were normotensive. It wasn't until the FTC intervened that the app was removed for making false claims.... From my perspective that's just the tip of the iceberg, because when you look at these apps online, there's very little information about the evidence that supports their claims.... Often times there are claims being made that are just unsupported."

Dr. Michael Hodgkins, Vice President and Chief Medical Information Officer, American Medical Association

It also offers to take the burden of logging health values or behaviors off the patient as a wearable sensor can collect, trend and analyze these. For instance, a 
connected weight scale, used regularly, can show a sudden weight gain in congestive heart failure patients that would indicate fluid retention and worsening health. If alerts and notifications were built to pop up in the treating physician's workflow (or sent to another caregiver), such data might be acted upon more rapidly or taken more seriously than patient self-reported values.

Exhibit 9: Measures Tracked by Connected Sensors

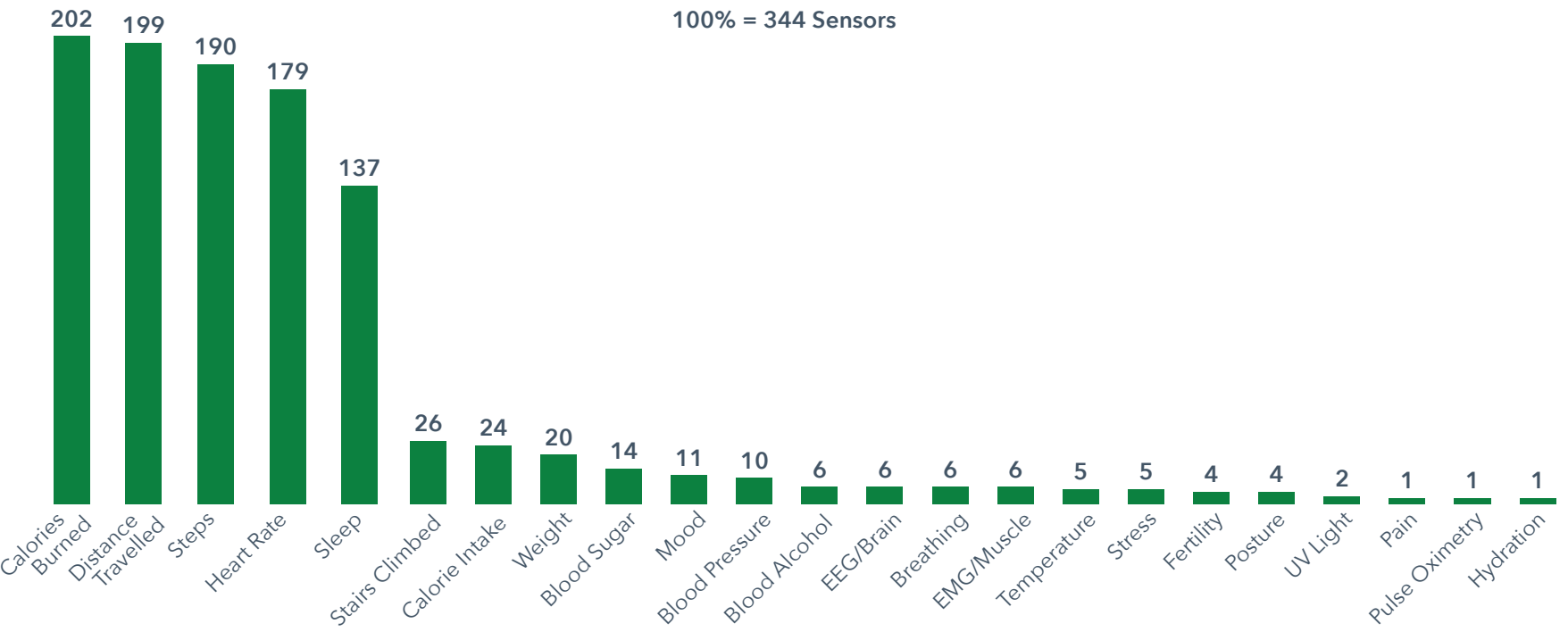

Source: IQVIA AppScript Device Database, Sep 2017; IQVIA Institute, Sep 2017

Note: Chart includes data from 344 sensors. Total measures exceed 344 due to multiple measures being tracked by a single sensor.

"Really, where the value is coming in right now, where the changes are being made, is in the sensors that are collecting the data. It's not just 'submit how you feel or submit how much pain you're in', et cetera... right now people are looking at the actual empirical data [from sensors]... They look at the [sensor] data first, and then they look at the patient-reported data to put context around it, but they're not looking at patient-reported outcomes on their own. That probably gives you a sense of where the trust falls."

Marc Sebes, Vice President of Product Management, Validic 


\section{Innovative uses of sensors}

- Digital sensors are transforming analog medical devices like asthma inhalers and injectable insulin pens into "smart" medical devices able to track usage and encourage patient adherence.

- New "digital biomarkers" of disease are being developed to track patient outcomes and improve the utility of medicines.

- Precision medicine will be supported by Digital Health tools enabling stratification of patients by symptoms and health behaviors; these are already being used to identify patients relevant for treatment in clinical trials as well as track disease severity in both clinical and trial settings.

- Digital tools are enabling the collection of "real world" data even within the bounds of clinical trials and offer a route to speed patient recruitment and retention, improve trial efficiency and create humancentered study designs.

Digital sensors linked to apps are bringing innovation in three key areas: encouraging adherence through the development of smart devices; improving patient care through the development of digital diagnostics, and transforming clinical trials by enabling new humancentered study designs.

\section{SMART DEVICES ENCOURAGING ADHERENCE}

Several clusters of innovation are appearing in this space. Devices like asthma inhalers and injectable insulin pens are evolving into "smart" medical devices with attached or built-in digital sensors capable of measuring and tracking usage and adherence programs are being wrapped around these. In the asthma space, pharmaceutical companies have increasingly partnered with, and even bought, leading adherence app and device developers to target the $30 \%-70 \%$ of asthma patients who are non-adherent (see Exhibit 10).15,16
These devices open up the possibility of alerting physicians to the need to alter therapy when a patient is overusing rescue medicine or dosing preventer medications incorrectly - offering to reduce exacerbations and improve clinical outcomes ${ }^{17}$ - while further generations of smart inhalers will also be able to determine if the full dose was received.

\section{ENSURING CORRECT USE IN ASTHMA}

Although first generation smart devices focus on adherence, sensors on next generation smart asthma inhalers may additionally show whether the patient took the medicine correctly and received the full dose (i.e., correct use), helping to adjust a patient's inhalation technique. For instance, the INhaler Compliance Assessment (INCA) device uses time stamped acoustic recordings of an individual's inhaler use to determine peak inspiratory flow rate and tell whether the device was used properly. ${ }^{18}$ Although initial research was done using the Diskus inhaler as a prototype, INCA-like technology is currently being applied to four new inhaler devices in a clinical trial. ${ }^{19} \mathrm{~A}$ similar acoustic sensor is being used in clinical trials by Sanara Ventures where "The acoustic sensor transforms airflow to a varying pitch whistle sound. The sound is recorded on a mobile phone and using proprietary algorithms, calculates peak flow and total lung capacity, FEV1 and other spirometric values." ${ }^{20}$

With insulin having rapidly become a commodity in recent years, companies are similarly looking to digital tools to provide differentiation in the diabetes marketplace. This has led to the rapid development of connected pens and pen caps for diabetes, which integrate a patient's usage of insulin into medical mobile apps to provide a record of when they used insulin, and for some, how much insulin they used. While the initial products' intent is to drive adherence - less than 
Exhibit 10: The Emerging Smart Inhalers Market

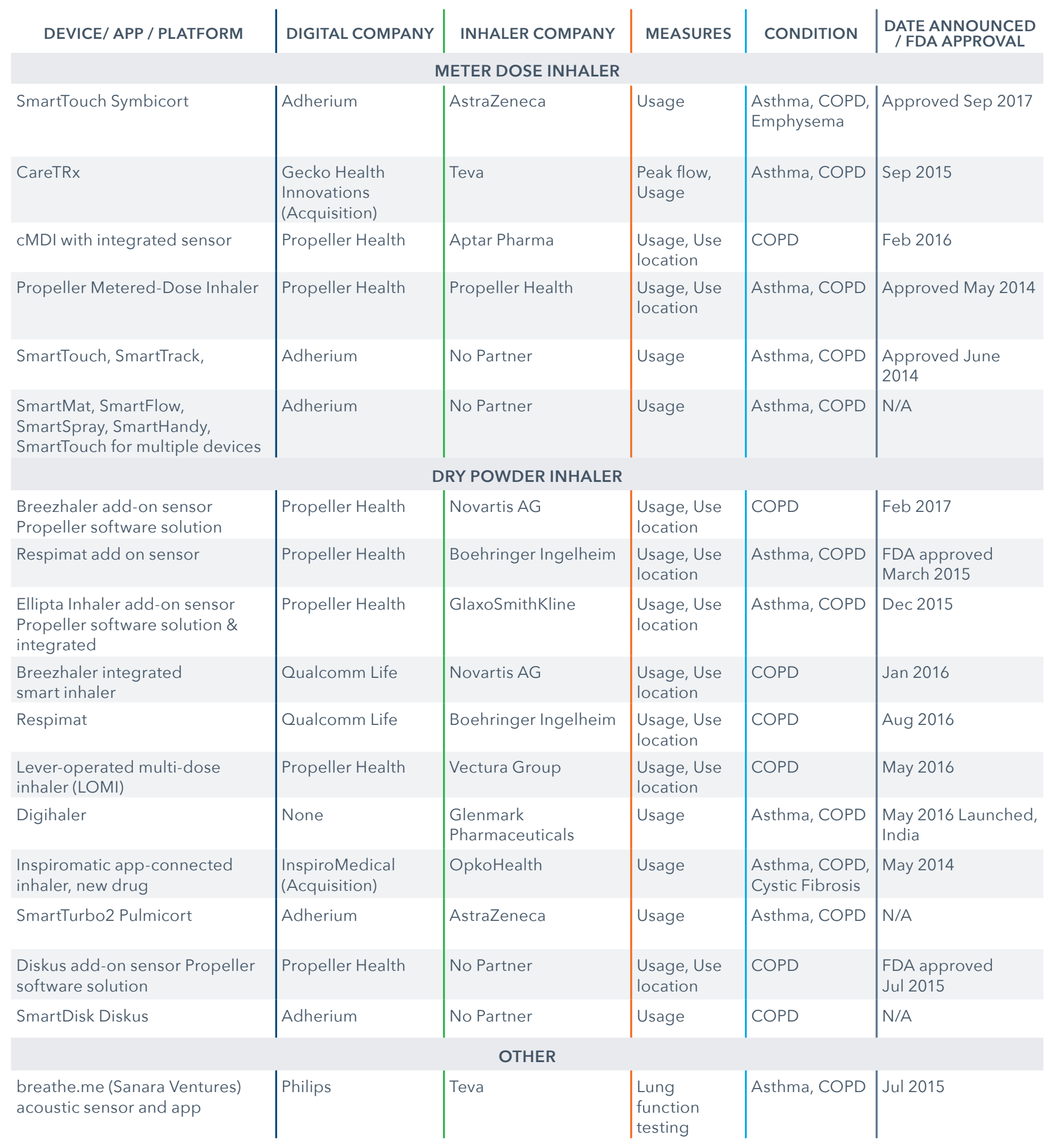

Source: IQVIA Institute, Sep 2017

Notes: Usage may include date and time the inhaler is used, how frequently, number of times per device and history of patient medication usage patterns. All devices include data transmission to a mobile platform and may include adherence reminders. 
$65 \%$ among insulin users ${ }^{21}$ - by linking to glucometer readings along with self-reported food intake data, a connected insulin pen could help users titrate their insulin usage more automatically. Companies working in this area include Eli Lilly with Companion Medical and Sanofi with Common Sensing, with other solo players Innovation Zed, Patients Pending and Emperra. ${ }^{22}$

\section{DIGITAL DIAGNOSTICS GUIDING CARE}

Parameter specific measurements from connected devices like blood pressure cuffs provide clinically meaning descriptors about human health. However, in order for the data collected from activity trackers and other consumer wearables to be useful to healthcare stakeholders, data scientists must uncover how variations in these digital measures (e.g., rotation, GPS movement through space, etc.) can track human health

\section{WHAT ARE DIGITAL BIOMARKERS?}

Digital biomarkers are the use of connected sensors, wearables and mobile devices and their capture of biomarker data (any substance, structure, or process of the body, its actions or products) to predict the incidence of disease or health conditions, track aspects of these, or assess the effects and value of treatments or interventions. These can include both user generated physiological and behavioral measures collected with the built-in sensors and processing units of digital devices (such as smartphones, for example). ${ }^{24}$ The value of digital biomarkers derives from their ability to be passively and continuously tracked in real time to explain, influence or predict health related outcomes. The development, validation and application of these new digital biomarkers is the focus of much activity among Digital Health developers and pharmaceutical companies, contract research organizations (CROs) and even health systems, as they may provide new tools to influence human health.
"This is the next wave of personalized medicine. It is about segmenting patients at its core. For both digital health and genomics, it's about delivering the right treatment, to the right patient, at the right time. The challenge is to see what we can measure with digital technologies, develop the algorithms to find interesting patterns and correlations for the data collected and then understand how to marry them. Then we can apply this combination of technologies and algorithms to personalize medicine."

\author{
Nelia Padilla, Vice President, \\ Advisory Services, IQVIA
}

status and outcomes. They must be translated using algorithms into new "digital biomarkers" of health that can then help healthcare stakeholders "identify the right patient and the right time for a given traditional intervention like a pill or a device as well as track the worsening or improvement of disease." ${ }^{23}$ In this way, the human data science of digital phenotyping will enable personalized medicine. 
Exhibit 11: Examples of Digital Biomarkers

\begin{tabular}{|c|c|}
\hline RHEUMATOID ARTHRITIS & $\begin{array}{l}\text { Using wrist-worn wearables to measure severity (e.g., by tracking steps as a proxy of activity levels } \\
\text { in the hours after waking). }\end{array}$ \\
\hline $\begin{array}{l}\text { PARKINSON'S AND } \\
\text { ALZHEIMER'S DISEASES }\end{array}$ & $\begin{array}{l}\text { Early detection of disease, associated dementia-related agitation and disease progression using } \\
\text { motion biomarkers. }{ }^{25} \mathrm{~A} \text { recent published study by Roche and Prothena Biosciences successfully } \\
\text { used a smartphone app in a Phase I Parkinson's trial to continuously measure symptoms including } \\
\text { tremors, balance, gait, standing up/sitting down transitions and turns, }{ }^{26,27,28} \text { while apps to detect } \\
\text { vocal tremor are also being tested. }{ }^{29}\end{array}$ \\
\hline MENTAL HEALTH & $\begin{array}{l}\text { Developing algorithms to detect digital behavioral markers of mental health phenomena } \\
\text { including depression and social anxiety from smartphone sensor data. }{ }^{30,31}\end{array}$ \\
\hline $\begin{array}{l}\text { ABILITY TO WALK IN } \\
\text { MULTIPLE DISEASES }\end{array}$ & $\begin{array}{l}\text { Tracking disease progression through gait changes or digital biomarker versions of the "six } \\
\text { minute walk test" (e.g. in Alzheimer's disease, juvenile fibromyalgia, geriatrics, heart failure, } \\
\text { multiple sclerosis, } \\
\text { stroke). }\end{array}$ \\
\hline EPILEPSY & $\begin{array}{l}\text { Using wearable devices for seizure detection and seizure counting. Sunovion is using the } \\
\text { Empatica Embrace wearable in a Phase IV clinical study of the seizure drug Aptiom, and Poole } \\
\text { Hospital in the United Kingdom is using the Microsoft Band similarly. Patients can potentially use } \\
\text { the devices to alert their kin or caregivers. }\end{array}$ \\
\hline MIGRAINE & Identification of patients having a migraine or about to have a migraine. \\
\hline
\end{tabular}

A number of areas have been initial targets for the development of digital biomarkers that may impact disease tracking and treatment (see Exhibit 11). Measures of mobility/motion, sleep and sleep disturbances - tracked well by wearables - have been put forward as indicators of disease severity in every major disease category.

Being able to determine the progression of human disease in a real world setting using digital biomarkers combined with predictive analytics - can enable case managers, physicians and payers to intervene for their patients in the real-world setting and offer patients the possibility of receiving help when and where they need it. ${ }^{36}$

Potential uses of digital biomarkers in the medicines space include:

\section{- Identification of patients relevant for treatment,} medicine-use, or for inclusion in clinical trials using digital biomarkers in addition to traditional biomarkers (e.g., blood tests). Typically these are used to narrow treatment to a subset of a drug's on-label population who obtain disproportionate outcomes benefit ${ }^{23}$

- Determining the efficacy of a drug using digital biomarkers to track severity in Phase IV trials or use them as primary or secondary endpoints in earlier trials

\section{- Gaining a better understanding of patient} experience when using a drug and measuring it in a more objective fashion than with patient reported outcomes in clinical trials or in a real-world setting

- Engaging patients once a drug is on the market; for instance, using biomarker data in a de-identified fashion to communicate with potential customers. This may include messaging to promote adherence or commercial applications like the presentation of ads 
Exhibit 12: Validation Trials per Fitbit Measure

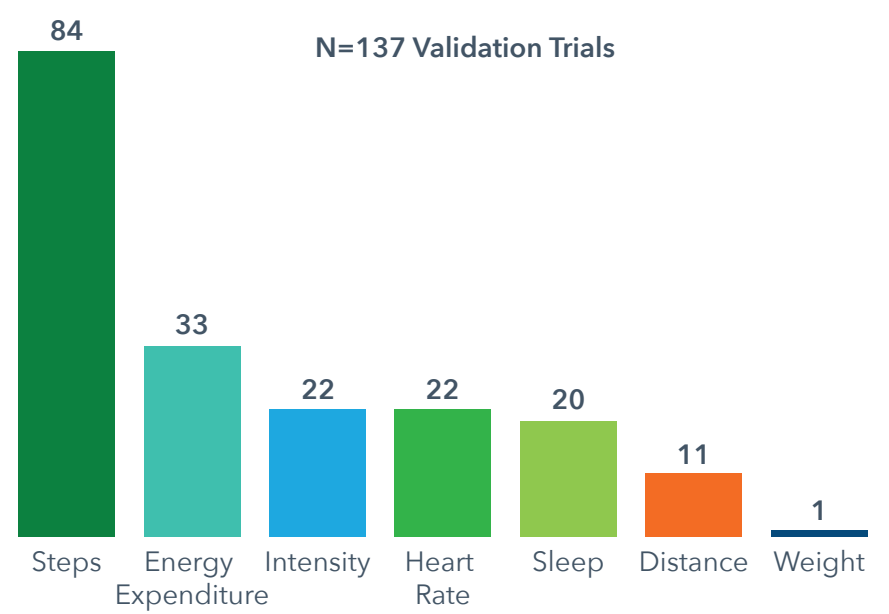

Sources: Fitbit Research Library Jul 2017; IQVIA Institute, Jul 2017 Note: Trial publications used as a proxy for unique trials; 193 measures across 137 publications

"Validation is coming into place for digital biomarkers. Digital biomarkers that are sensorbased need to be validated in order to be rolled into a clinical trial and need to be validated in order to really change physician prescription behavior for diseases that are asymptomatic. Lots of folks are trying to generate... evidence for their digital biomarkers right now, too."

Christine Lemke, President, Evidation Health
"I can put them on medication and I can start to see the efficacy of that drug [with a digital biomarker]. We've been relying on patient-reported outcomes but now, we can actually get some empirical data that shows what the results really are and even match those up to the patientreported outcomes to see how accurate those outcomes have been, or how accurately they've been reported."

Marc Sebes, Vice President of Product Management, Validic

Assessing the value of wearable sensors to provide clinically meaningful data in the form of biomarkers is a necessary step to obtaining value across the domains tracked by those sensors. Although this process of validation began only in the past few years, there have now been 137 trials focusing on validation for Fitbit devices alone (see Exhibit 12).

Companies active in the Digital Health space are actively validating digital biomarkers and other Digital Health tools, with clients that include sensor developers, startups, pharmaceutical companies, payers and healthcare systems alike. For example, Evidation Health 
suggests that in just one year from 2016-2017, the percent of its clients seeking to test device- or sensorbased biomarkers grew from $20 \%$ to $50 \%$, with other clients running evidence testing for apps and programs. These trials seek to generate evidence that the digital biomarker measures what it claims to, and that it correlates to accepted clinical endpoints. ${ }^{36}$ This is critical since some studies have shown that although consumer wearable activity trackers may provide comparable results to clinical actigraphy devices on some parameters (i.e., step count, activity, sleep start times, sleep duration) they can be less precise or overestimate other tracked elements such as sleep duration, vigorous physical activity or total daily energy expenditure. ${ }^{37,38}$

\section{REGULATING DIGITAL DIAGNOSTICS}

The fact that digital biomarkers may be built on top of a phone or sensor to harvest data makes them susceptible to some variability over time; for instance, when a company does a software update or as hardware evolves. Without testing, it is unclear what happens to the value of a digitally collected biomarker as technology evolves. Accuracy and reproducibility could be compromised. The FDA is reported to be working towards an approach to ensure consistency of digital biomarkers - a significant hurdle. The process of digital biomarker validation is expected to be distinctive from traditional diagnostic, medical device or therapy approval processes - allowing some permissiveness to be built into the validation, and yet sufficient for industry, payers and physicians to accept these as valid measures for understanding therapy impact and making diagnosis and therapy decisions. ${ }^{36}$

Digital diagnostics that measure existing biomarkers already accepted by the medical community, such as bilirubin, respirometry measures, or blood glucose, have a straightforward regulatory pathway to prove accuracy via the $510(\mathrm{k})$ pathway, evaluating technical and substantial equivalence to existing tools. However, to the extent that digital diagnostics developers are not only proposing a new measurement modality, but also an entirely new "biomarker" to measure for purposes of diagnosis, the FDA is likely to create a high bar for approval for clinical use. To gain FDA clearance for such first-in-class tests, developers will likely have to prove to the FDA that the "digital biomarker" displays not only technical accuracy, but clinical accuracy and utility in terms of acceptable sensitivity (i.e., few false negatives), specificity (i.e., few false positives), and improvements for human health care (e.g., early detection of disease or better treatment recommendations). This effort will likely require the use of the FDA's Pre-Market Approval (PMA) process which is a higher bar than the 510(k) process most apps and devices have used to-date and almost always requires supporting clinical data.

In either case, when many software updates are required to carefully hone required algorithms, or for software used as a medical device, the FDA's new Digital Health premarket certification program (Pre-Cert) may also become increasingly useful and popular. This program will focus on certifying firms and developers, which once declared responsible and safe in their development won't be required to run each product and product update by regulators. Still in pilot stage, the FDA announced in September 2017 that nine companies including Apple, Samsung, Google, Johnson \& Johnson, Roche, Fitbit, Phosphorus, Tidepool and Verily were selected to be part of the pre-certification pilot process, indicating a variety of parties with differing business models - large tech companies, traditional medical device manufacturers and small entrepreneurs - are interested in taking Digital Health to the next level. ${ }^{39,40}$ 


\section{TRANSFORMING CLINICAL TRIALS}

Pharmaceutical and biotech manufacturers together with $\mathrm{CRO}$ s are at the early stages of understanding how to best leverage Digital Health technology in clinical development but have naturally experimented with it as they look for ways to speed enrollment into clinical trials, reduce costs, cycle times and inefficiencies, and enhance patient retention. Manufacturers are exploring the use of Digital Health tools, also, to generate and track new outcome measures such as digital biomarkers and patient experience metrics, to provide them some differentiation in the market.

\section{Recruitment}

One of the key challenges and major cost drivers of clinical trials is recruiting patients in a timely fashion; particularly for large Phase III/IV trials, where 1,000 to 5,000 patients may be enrolled per trial ${ }^{41}$ and costs average $\$ 20$ million. ${ }^{42}$ Not only can incorporating Digital Health into trial designs ease the burden of patient participation, but more importantly, leveraging telehealth solutions to screen patients increases the number of potential patients screened, and therefore can speed up enrollment rates. Many patients live far from academic medical centers and other hospitals where clinical trials are typically carried out. By screening patients via telehealth, not only do sponsors reduce the costs associated with setting up locations that never enroll patients, but patients save time and money by not having to travel to the clinical trial site only to be told they are not eligible for the trial. The enrollment process can be further sped up by obtaining electronic consent of patients using e-signatures or other interesting technologies like virtual reality for pediatric patients.

The behavioral and lifestyle information from digital consumer tools, used passively by individuals in the real world, can also be combined with predictive analytics to speed the selection and recruitment of patients. Databases of individuals who consent to make their data available for research, including those run by IOVIA (i.e. Mediguard), Evidation ${ }^{43}$ or the Precision Medicine
"Recruitment can be much faster when you know and have a well characterized population, with a history of sensor data combined with traditional medical data, and you don't need to wait to find people. Enrolling people tends to be the thing that draws out a trial... and drives the great part of expense and time...We found that we can shorten the recruitment enrolling processes by ten times. We can do it ten times faster based on a well characterized population and based on our ability to reach out to them directly."

Christine Lemke, President, Evidation Health

Initiative Cohort Program (PMI-CP), provide a wellcharacterized population or "pool" of individuals for inclusion in clinical trials. Researchers can infer traits pertaining to health and wellness behaviors, such as levels of patient activation, to recruit individuals more likely to have continued participation in clinical trials. Researchers can infer traits pertaining to health and wellness behaviors, such as levels of patient activation, to recruit individuals more likely to have continued 
participation in clinical trials. They can also potentially leverage digital biomarkers in the future to identify patients relevant for treatment.

\section{Human centered study designs to improve retention} Human centered study designs enabled by Digital Health offer the possibility of increasing patient participation and retention. Digestible sensors, wearables and companion smartphone apps enable direct-to-patient interactions and virtual engagement. Allowing patients to complete study procedures using an iPad for data entry, participate in FaceTime or telemedicine visits rather than onsite visits, and perform diagnostic tests using connected biosensors, all make it easier for patients to participate. ${ }^{44,45}$ Relieving the burden for patients to make multiple excursions to clinical trial sites for testing - to the extent possible in clinical trials of long duration or providing more dynamic patient support through digital tools, can help prevent them from dropping out or discontinuing therapy. ${ }^{46}$ One example is the use by AstraZeneca in 2016 of a companion smartphone app (by Voluntis) for patient support in several ovarian cancer treatment trials. The app helped clinicians better manage therapy side effects of hypertension and diarrhea, and thereby reduce therapy discontinuation and minimize dose modification . ${ }^{47}$

\section{Improving adherence}

Other areas of clinical trial inefficiency are also targets for improvement by Digital Health, such as the 20-40\% rate of medication non-adherence, where patients may not take study medicine consistently or at the right time of day. ${ }^{48,49}$ In Phase I pharmacokinetic studies, consistency in dosing is a key factor in the success of a product. Digital health adherence tools like the AiCure mobile app, Pillo or "smart blisters" (described above), may allow a way to control dosage time of day and are gradually being incorporated into protocols to improve clinical success. Some barriers exist to the use of photoimaging technologies like AiCure, however, with some IRBs and patient advocates conservatively expressing concern around adherence driven by patients being imaged. ${ }^{46}$ Other technologies, such as Pillo, which use facial recognition scanning without recording images are emerging to address these concerns. In Phase II-IV trials, adherence to trial protocols and regimens are similarly critical, and mobile apps can be deployed to push out protocol reminders to participants.

Mobile data collection for product differentiation Perhaps most transformative is the incorporation of Digital Health tools into clinical trial protocols for the collection of human health data. This can include the collection of patient self-reported data from apps, or passive biometric measurements from sensors for the participants enrolled. Both can help provide information on patient experience in clinical trials that highlight elements that matter most to the patient and provide a way to gather data continuously. Patient reported outcomes (PRO) data (i.e., patient input on their perceived change in symptoms or wellbeing) is increasingly valued as measures of success for healthcare interventions as the healthcare system strives to be more patient-centric. Digital versions of these metrics, or technology-enabled clinical outcome assessments (TECOAs) often deliver survey questions digitally, and are increasingly being incorporated into clinical trials. These may include the same quantitative survey instruments trusted by the medical community, or patient subjective assessments of mood or pain that may be more loosely defined (i.e., smiley face), though the former is more likely to be included in clinical trials. Validated PRO apps like the Cleveland Clinic Multiple Sclerosis Performance Test (MSPT) iPad disability assessment tool can be used for measurement of neurological and neuropsychological impairment and disability in multiple sclerosis - a precise, valid measurement of MS severity.

Sensors are also being incorporated into research to track human health outcomes, offering greater objectivity versus traditional survey methods. In traditional trials patients might be required to fill out daily diaries or assessments by paper recording their 
sleep patterns, but sometimes do so two weeks later just before the next in-person visit when their ability to recall may be diminished. Wearables, on the other hand can collect information real-time rather than just delayed data, thus avoiding patient recall bias, and are often well-accepted by patients due to the unobtrusive fashion of data collection. ${ }^{50}$ Tracking sleep using a wearable (e.g. Actigraph or other actigraphy device) may serve as better proxy of how well a person is sleeping than patient self-report. ${ }^{51}$

Continuous or frequent biometric measurements (i.e., blood pressure, activity, etc.) can also be recorded throughout the day with sensors as patients go through their normal routine rather than keeping them in a clinic for an extended period. This offers the ability to track therapeutic outcomes in more naturalistic setting to provide "real world" evidence (see Exhibit 13), even within the bounds of a clinical trial. Patient outcomes and behavioral data can also be tracked for a longer time after a therapeutic intervention ends using wearables - by linking to claims data from data providers like IOVIA or PokitDok ${ }^{52}$ to track patients in an opt-in fashion and in open-label studies - thus providing additional real-world outcomes evidence.

While detractors say consumer wearables are not yet fit for purpose and few have the right sensitivity or specificity to measure human health outcomes compared with clinical wearables, such devices offer new ways to provide value. New algorithms and biomarkers specifically written and validated on these devices offer an approach to obtaining value from consumer wearables rather than force fitting old endpoints into what wearables can provide. ${ }^{53}$

While using Digital Health tools may enable data to be gathered more frequently (sometimes in real time, often multiple times per day) and at a very high quality to improve trial efficiency and reduce costs of clinical

Exhibit 13: Digital Health Use in Clinical Trials for Patient Monitoring

\section{Real Time Data Received}

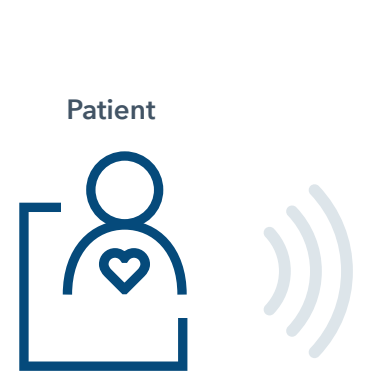

Monitoring

Analytics and Visualizations

Digital Health Tools

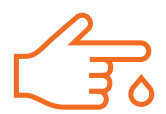

Continuous Glucose Monitoring

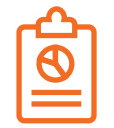

Electronic Patient Reported Outcomes (ePRO)

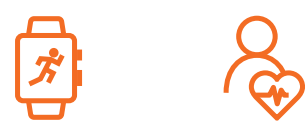

Electrocardiogram (ECG)

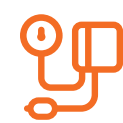

Blood Pressure Monitor
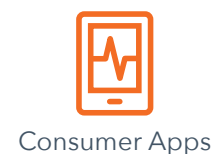

Consumer Apps Monitor
Investigator

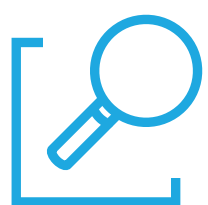

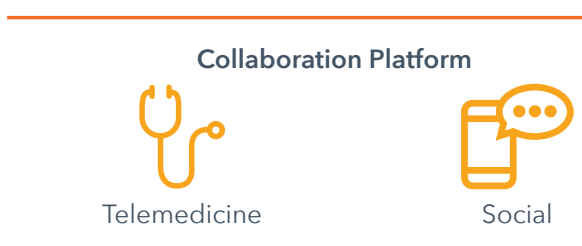

Source: IQVIA, Jun 2017 
time, the cost implications are not unidirectional. Large volumes of complex data provide the added burden and cost of collection and processing, including data cleansing requirements, analytical time, validation, automated reviews and the building of infrastructure to

"On the pharmaceutical side... there's certainly a lot of experimentation going on during the trial process and before the trial process, but where we're seeing things start to actually happen is after the drug is out there [on the market]. They are saying, 'Let's have more interaction with this patient and how they take the medication. Let's learn more about the real world use of this drug while it's out in the wild ... rather than jump straight to the more challenging aspect - at least from a regulation side of how to incorporate all this into a[n approval] trial..."'

Marc Sebes, VP of Product Management, Validic support these activities. ${ }^{46}$ For instance, in a Parkinson's disease biosensor study, wearables generated unique outcomes data; however, a total of 24,104 hours of passive monitoring data was generated through the course of the study. ${ }^{18}$

Companies are approaching the use of Digital Health in clinical trials cautiously, trying to better understand how to use it effectively. Due to the risk for companies incorporating these new measures into approval trials, where a misguided application of Digital Health tool could jeopardize a drug approval, most trials are incorporating these as exploratory endpoints for commercial Phase IV trials on marketed drugs, where risk and regulation are lower. Only a small number of manufacturers have taken the step to use digital tools with new drugs or for gathering/generating primary and secondary endpoints. Most companies are running proof of concepts with sensors like Fitbit to learn how best to incorporate them into clinical trials. ${ }^{53}$ These act as testing grounds for the company to learn what infrastructure is needed, what governance structure, and what data strategy is needed to incorporate digital technology into a protocol, including aspects of privacy and security, and how to handle adverse events (AEs) that might be recorded.

Finally, guidelines for the use of digital technologies are emerging to guide endpoints and ensure they are valuable and meaningful. The Clinical Trials Transformation Initiative (CTTI), a public-private partnership of pharmaceutical companies, academics and regulators, including the FDA, released guidelines in June 2017 for selecting novel endpoints. These focus on practical approaches to developing endpoints, and suggest only using novel mobile technology-derived assessments and endpoints when they offer incremental value over existing standard measures, including providing greater meaning to patients. ${ }^{54}$ 


\section{Delivering value to patients and the health system}

- High-quality apps - those with characteristics such as high patient ratings, frequent updates, connectivity to sensors, inclusion in Digital Health formularies, endorsements, and promising clinical evidence now exist for each major type of healthcare use.

- The overall body of clinical evidence on app efficacy has grown substantially and now includes 571 studies, including 234 randomized controlled trials and 20 meta-analysis studies.

- Particularly strong evidence now exists for diabetes, depression and anxiety that may be considered by clinical guideline writers (e.g., the American Diabetes Association, the American Psychiatric Association) for incorporation into standard of care recommendations.

- The strengthening maturity of clinical evidence in diabetes, cancer, post-traumatic stress disorder (PTSD), arthritis, stroke, genitourinary conditions, pulmonary rehabilitation and dental uses, has been significant over the past 3 years, with new studies showing significant benefits vs. controls; however, exercise, autism and bipolar disorder experienced disappointing study results.

- Of the top 25 apps, $80 \%$ have at least one positive observational study demonstrating clinical efficacy, over half connect to an external sensor, one quarter are not publicly available to patients and one fifth are FDA-cleared.

- The use of Digital Health apps in just five patient populations where they have proven reductions in acute care utilization (diabetes prevention, diabetes, asthma, cardiac rehabilitation and pulmonary rehabilitation) would save the U.S. healthcare system $\$ 7$ billion per year and provide tangible outcomes improvements.
- Extrapolating this level of cost savings approximately $1.4 \%$ of total costs - to the entire U.S. health system, would yield total U.S. annual cost savings of $\$ 46$ billion.

\section{VALUE ALONG THE PATIENT JOURNEY}

A large supply of apps exists for nearly every use across the patient journey. However, Digital Health apps will ultimately be judged - like any other medical technology - not by their quantity but by their value to human health and the healthcare system. Value in healthcare can be defined as the health outcomes created for patients relative to the cost of generating those health outcomes, ${ }^{55}$ as well as the extent to which the "triple aim" of improving the patient experience, improving the health of populations, and reducing the per capita costs of healthcare is delivered. ${ }^{56}$

Digital Health apps have a range of potential mechanisms for delivering value. To patients, Digital Health apps are typically seen as delivering convenience. One survey of 1,000 U.S. adults found that the top three capabilities patients wanted from apps provided by their healthcare providers are (1) digital access to medical records, (2) appointment management and (3) prescription refills. ${ }^{57}$ Among capabilities offered by the Walgreens app, patients have adopted digital prescription refills and have begun to adopt telehealth capabilities such as chatting with pharmacists and virtually visiting physicians via MDLive. ${ }^{58}$

Physicians, on the other hand, often see the value of Digital Health apps in terms of spurring patient engagement, promoting more efficient providerpatient communication, and creating new modalities for condition diagnosis and monitoring. A poll of 1,300 U.S. physicians by the American Medical Association (AMA) found that physicians are most attracted to Digital Health tools to improve work efficiency, increase patient safety and improve diagnostic ability. The same survey found that $26 \%$ and $13 \%$ of physicians were currently using 
Digital Health tools to promote patient engagement and for remote monitoring, respectively. ${ }^{59}$ These are the same areas a poll of 595 members of the NEJM Catalyst Insights Council - which is made up of healthcare executives, clinical leaders and clinicians - showed to be the top benefits of using patient engagement tools: supporting patients in efforts to be healthy $167 \%$ of respondents) and providing input to providers on how patients are doing when not in clinic (60\%). 60

Patients and providers seek value from Digital Health across the full spectrum of the patient journey (see Exhibit 14).

Wellness and Prevention apps and their connected sensors support patient efforts to set health goals (e.g., losing weight), track daily lifestyle changes (e.g., reducing calories), and monitor their progress achieved (weight loss vs goal). The value of such apps is associated with the mitigation of risk factors, such as obesity or smoking, which impact longterm costs to the health system. Payers with budget responsibility for a given patient over a multi-year time frame, such as the Medicare program and self-insured employers, therefore stand to benefit the most from these apps, and sometimes incorporate them into incentivized wellness programs. While Wellness and Prevention apps have become extremely popular, with most downloaded by individuals independently of a physician's guidance, physicians are comfortable recommending these apps to patients given the low perceived risks to patient safety, limited expectations for clinicians to take time to review data and high availability of good free and publicly available apps. Under Armour alone - the owner of the popular MyFitnessPal, MapMyFitness and Endomondo apps claims more than 200 million registered users with 100 thousand new users signing up each day. ${ }^{61}$

Patient Experience Tools help patients manage their interactions with the healthcare system via new digital channels that offer added convenience. Whether providing access to health records or scheduling appointments (either for physical in-person or virtual

\section{Exhibit 14: Digital Health in the Patient Journey}

\section{Wellness \& Prevention}

Wellness \& Prevention
Exercise \& Fitness
Diet \& Nutrition
Lifestyle \& Stress
Stress Management
Sleep/Insomnia
Smoking Cessation
Alcohol Moderation

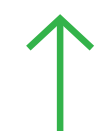

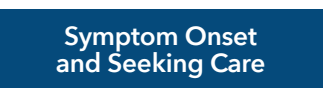

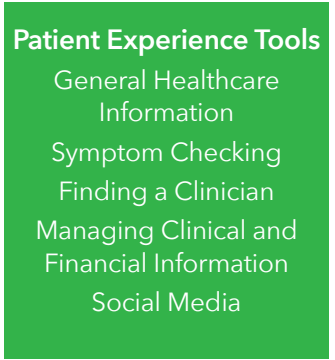

Managing Clinical and

Financial Information
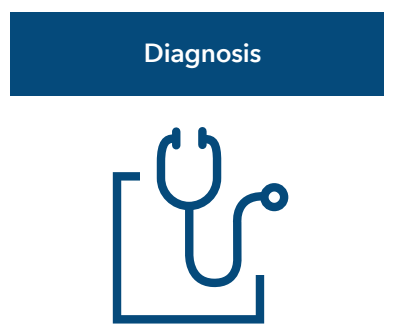

Physician may recommend app-supported disease management programs connected sensors for remote monitoring, or apps for any use case across the patient journey

\section{Condition Monitoring}

Condition Education

\& Management

Self-Monitoring

Remote Patient

Monitoring

App-Enabled

Rehabilitation Program
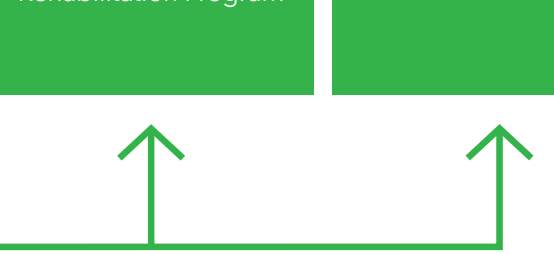

\section{Treatment}

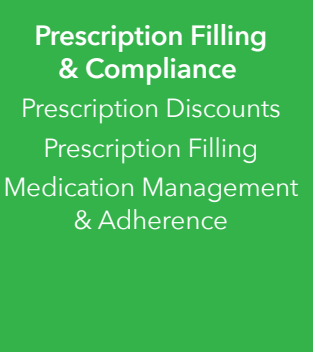

Prescription Filling

Prescription Discounts

Prescription Filling

Medication Management

\& Adherence

Patient Journey

Digital Health Use Categories

Source: IQVIA AppScript Use Categories. IQVIA Institute, Sep 2017 
telehealth visits), the value of these apps is in their ability to make the experience of healthcare easier and more satisfying for patients while promoting provider efficiency. Many of these tools have seen significant adoption in recent years as new regulations that have spurred the development of online patient portals and other systems, core business interests and the availability of high-quality tools have come into alignment with patient needs. The fact that tools are generally not specific to any particular condition but are applicable to most patients, has also helped adoption. Some examples of these tools include:

- Symptom Checkers: An increasing number of apps provide potential ailments based on a list of patient provided symptoms. These apps can be used to selftriage and make decisions around healthcare needs, such as whether to visit a physician or head to an urgent care clinic. Many of these apps are currently deployed by digital publishers (e.g., WebMD) and are therefore direct-to-consumer, ad-supported products. This being said, anecdotal reports suggest that a new generation of such apps may seek to reduce unnecessary visits, particularly to emergency departments, creating a more tangible opportunity for traditional healthcare stakeholders (e.g., payers).

- Healthcare Professional (HCP) Finders: Patients often struggle to find an adequate physician that takes their insurance and is available at a convenient time and location. Tools like ZocDoc have had considerable success in serving these needs. New trends in this category include sophisticated cost comparison tools (e.g., Castlight Health) and an increasing interest by large health systems in better matching patients to their sprawling networks of sub-specialists.

- Managing Clinical and Financial Records: Patients have traditionally encountered issues accessing, organizing and transmitting health records (e.g., vaccination records). So-called "Meaningful Use" regulations (now integrated into new "MACRA" and
"We live in an age of chronic illness. It's very clear that you can't just manage chronic illnesses in the four walls of the clinic. You have to be able to reach beyond the clinic, to where the patient lives, where they work, their particular situation in terms of support, from family and others... as well as the social factors that greatly affect how chronic diseases can be managed effectively, and what the outcomes will be. You need these [digital] tools. You need tools that allow you to interact with patients at a distance. You need tools that allow you to monitor certain things at a distance. You just can't succeed by having the patient come to the clinic every so many months."

Dr. Michael Hodgkins, Vice President and Chief Medical Information Officer, American Medical Association, August 9, 2017 
"MIPs" schemes) require providers to supply a number of their patients with access to their health records, typically via "patient portal" apps. The result has been the improvement and uptake of associated apps (e.g. MyChart by Epic).

\section{Condition Education and Management apps provide} condition-specific tools, often for daily use by those afflicted with the condition or their family caregivers. Patients with conditions - particularly complex, chronic conditions - often need tools that provide appropriate education on how to perform new tasks (e.g., measuring blood glucose), enable them to store notes (e.g., blood glucose logs), and track progress towards goals (e.g., keeping blood sugar in range). The value of such apps is often measured in terms of traditional health outcome measures, such as improvements in laboratory test results (e.g., HbA1c) or avoidance of condition destabilization (e.g., hyperglycemia) leading to acute care utilization. To date, adoption of Condition Education and Management apps has been predominantly by patients and their caregivers for selfmanagement; however, some healthcare providers are beginning to incorporate remote monitoring of patientgenerated health data into their workflows.

Some examples of condition management tools include:

- Self-Monitoring Apps that help patients track their specific condition over time for their own personal use. One example is the Start app by lodine Labs (acquired by GoodRx) which helps patients monitor the efficacy and side-effects of new antidepressants. Users of the Start app are asked questions from the PHQ-2 and $\mathrm{PHQ}-9$ which are two scales used in depression. Users can see how their results change over time as an objective measure of whether their medication may be working. This information may help patients communicate their symptoms to clinicians at their next office visit, potentially accelerating the process of finding an appropriate antidepressant.
- Remote Patient Monitoring Apps that enable clinicians and population health case managers remotely monitor patient data. Although these can include apps and app-supported programs like Omada that are available to consumers, many of these are currently custom-built by health systems to meet their needs and integrate into their electronic health record (EHR) system. For example, Sutter Health, a large healthcare provider in Northern California, has leveraged Validic technology to push certain blood pressure and blood glucose data into its EHR enabling clinicians to review trends with patients when they visit. At least one Sutter Health pilot also used a hypertension care team to intervene with patients whose 14-day blood pressure trend appeared to be problematic. This population health team was able to make timely adjustments to medication dosage, exercise and diet that may have avoided the destabilization of certain patients.

The value of Digital Health to conduct and optimize remote patient monitoring is significant. By identifying patients with vitals or values out of range, and alerting the care team, Digital Health apps and sensors can narrow the number of patients a nurse or care manager has to call and prioritize their lists, providing a smarter route to providing continuity of care. By making care managers more efficient they can get relevant patients into the office sooner when there is an issue. Examples of such health interventions, along with physician recommendation of apps, are shown in Exhibit 15.

- App-Enabled Rehabilitation Programs, such as to cardiac and pulmonary rehabilitation patients, that reduce the requirement for patients to visit a physical place multiple times a week.

\section{Prescription Filling and Compliance apps help} patients find lowest cost drugs, refill prescriptions, encourage medication adherence via reminders, ensure polypharmacy is appropriately managed, and provide targeted education on medicines and dosing instructions. 


\section{Exhibit 15: Use of Apps, Sensors and Digital Health Supported Programs for Condition Management}

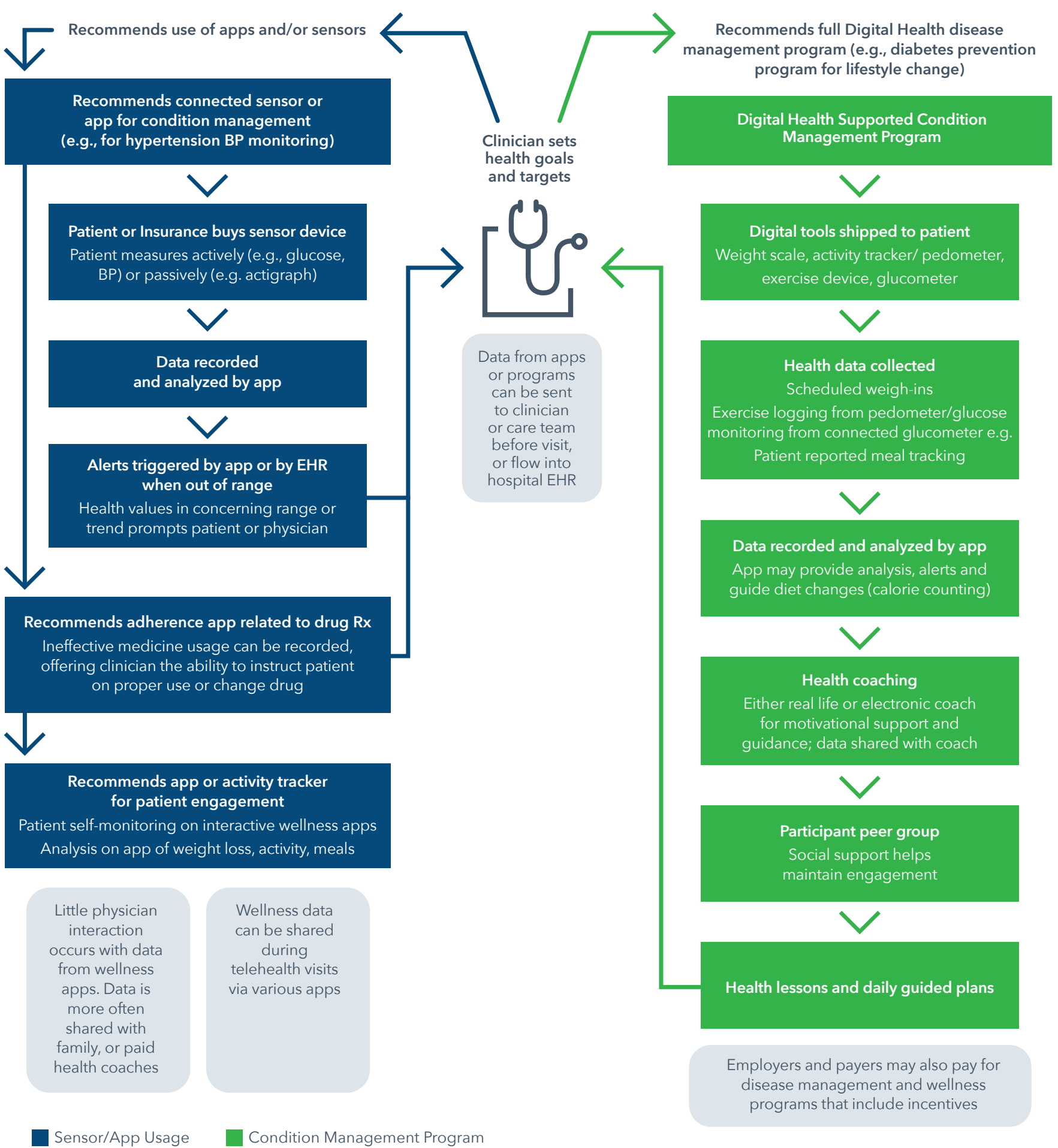

Sources: IQVIA Institute, Sep 2017; Quan R, Omada Health. Using Data Science to Design Effective Precision Preventative Behavioral Medicine. Slideshare, Nov 2016 Note: Health coaching may come from population health managers, care coordinators, health coaches, case managers and care managers 
They can also help mitigate the challenge families face to ensure an aging parent is correctly taking their medicines. Digital Health tools in this space that can enhance medication adherence and reduce acute care utilization offer a route to significantly impact healthcare costs. ${ }^{62}$ In that regard there is the potential for broad support from traditional stakeholders. For payers and at-risk health plans nonadherence is a key target for cost reduction, with as much as $\$ 105$ billion spent on avoidable costs in the United States in 2012 due to medication therapy nonadherence ${ }^{63}$ and $\$ 269$ worldwide, accounting for $57 \%$ of the world's annual total avoidable cost, ${ }^{64}$ while healthcare providers are focused on reducing acute care utilization. High levels of adherence are associated with lower overall health care costs for a number of chronic diseases including diabetes, hypercholesterolemia and hypertension where many medications are generic, as well as chronic obstructive pulmonary disease (COPD) and severe asthma. ${ }^{65}$

\section{A range of Digital Health tools have emerged to help} encourage adherence or even allow caregivers to track and ensure medicines have been taken. Among these are mobile apps, such as AiCure, that image and note patients taking their medications; digital "prescription compliance devices" for real time adherence monitoring, such as "smart blisters" (pharmaceutical packages capable of monitoring when a pill is taken out of its packaging) and smart pillboxes (e.g., Wisepill); 66 and ingestible sensors such as Proteus, ${ }^{67}$ all of which help control dosage time of day by reminding patients of, and alerting caregivers to, missed doses. Another mobile app, Medisafe, which pushes out medication reminders and alerts, showed it could increase cholesterol medication adherence by as many as three days of therapy per month. ${ }^{68}$ Mobile apps in this space have seen tremendous adoption. Google Play states that the Walgreens Android app has 10,000,000-50,000,000 downloads alone. The Medisafe and GoodRx Android apps both have 1,000,000-5,000,000 downloads.
"For us, we're not trying to push anyone into a particular channel. What we're looking for are ways to reduce friction. We have multiple studies which have indicated that the folks who are using our digital channel, [our apps and online experiences], see that it makes things faster, easier and more simple - as you would assume, based on the results of these studies. If you make things easier for people, they are more likely to engage! We end up with adherence lifts and increases across other key performance indicators for people who are using our digital channel."

Greg Orr, Vice President, Digital Health, Walgreens Boots Alliance

To deliver value for a given use in the patient journey, there must be at least one high-quality app available to support that particular need or use. One approach that has been developed to measure the quality of apps is the AppScript Score (see Exhibit 16 and Methodology section for quality rating methodology). 


\section{Exhibit 16: IQVIA AppScript Score Overview - A Way to Measure the Quality of Apps}

Rating Averages are Weighted to Create AppScript Scores

\section{Professional}

- AppScripts sent

- Professional ratings

- AppScript formulary inclusions

\section{Patient}

- App store ratings

- App store reviews

- Patient use metrics:

- AppScript fill rate

- AppScript retention rate

Functional
- Comprehensive functional
assessment of an app's ability to:
- Inform - Guide
- Instruct - Remind
- Record - Message
- Display

\section{Endorsement}

- Number of endorsing institutions

- Type of endorsing institution

\section{Developer}

- Use of advanced development techniques

- App update cadence

\section{Clinical}

- Number of studies

- Type of studies

- Outcomes of studies

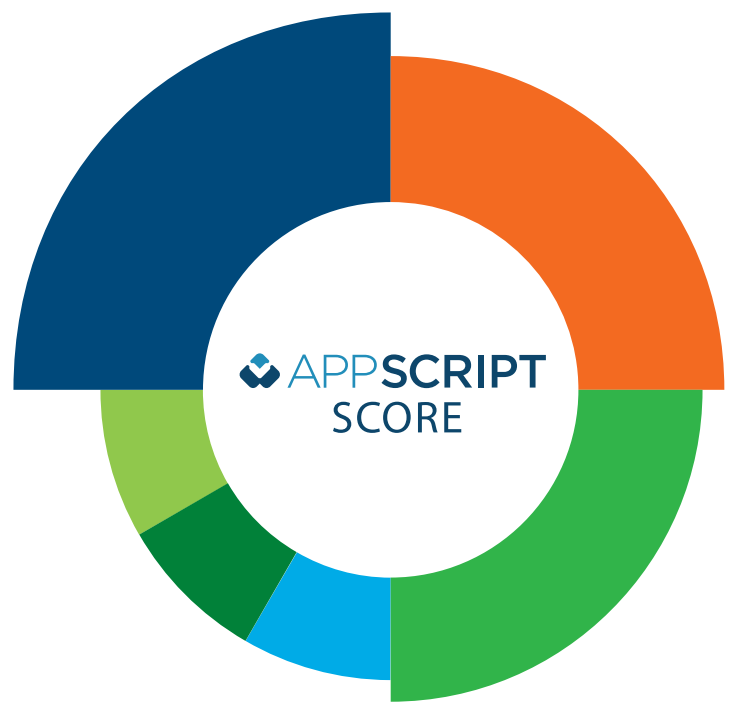

Sources: IQVIA AppScript, Aug 2017

While a large number of apps of varying quality are available in each category, most app categories have at least one high-quality app (with an AppScript Score >75) with key quality characteristics such as (1) exceptional patient ratings, (2) connectivity to sensors, (3) rapid update cadence thereby ensuring that apps are reliable and incorporate the latest technologies and (4) have endorsements from at least digital publishers and often from providers or government authorities (e.g., the FDA) (see Exhibit 17). While each category generally has at least one high-quality app, average app quality is often low. This implies that while high-quality apps exist, careful app selection is required to ensure quality.

\section{A GROWING BODY OF EVIDENCE}

Since 2007, the amount of peer-reviewed efficacy studies of Digital Health apps has grown dramatically. Of at least 571 efficacy studies published during this time period, a quarter $(n=138)$ have been published in 2017 year to date (YTD) alone as of August 14th (see Exhibit 18). Randomized controlled trials (RCTs) and meta-analysis studies, which are favored by those stakeholders making evidence-based medicine decisions, collectively make up approximately $44 \%$ of the studies. Since 2007, 234 RCTs and 20 quantitative meta-analysis studies have been published.

This acceleration in activity is driven by the intersection of a skeptical environment with robust private-sector fundraising, an increasing ability to leverage real world evidence to deliver studies faster and more economically, intense interest from leading academic institutions, and new journals dedicated entirely to the field (e.g., "JMIR mHealth and uHealth").

While the overall volume trend for Digital Health efficacy evidence appears impressive, adoption decisions are likely to be made one app use-category at a time. To this end, it is important to understand 


\section{Exhibit 17: Distribution of AppScript Score by Digital Health App Category}

Patient Experience

Use Case

General Healthcare Information

Find a Physician

Social Media

Managing Clinical and

Financial Records

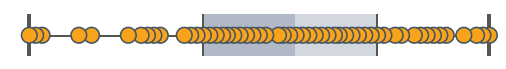

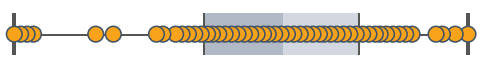

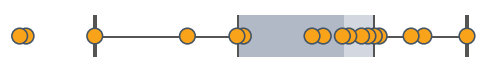

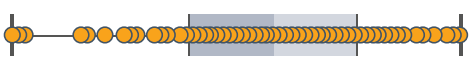

$20 \quad 40 \underset{60}{60} \quad 80 \quad 100$
AppScript Score
Wellness \& Prevention

Use Case

Exercise

Healthy Eating /

Weight Management

Stress Management

Drug \& Alcohol Abuse

Smoking Cessation

Vision \& Hearing

Sleep / Insomnia
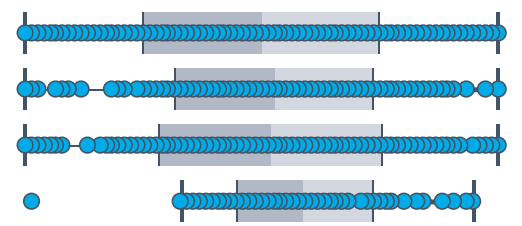

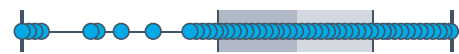

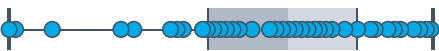

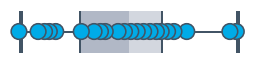

$\begin{array}{lllll}20 & 40 & 60 & 80 & 100\end{array}$

Use Case

Condition Management

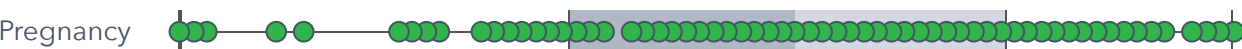

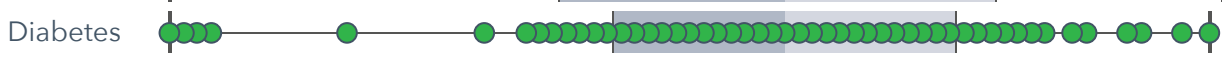

Prescription Filling \& Adherence $\phi 00 \_0-0-000-0-00000000000000000000000000000000000000000-\phi$

Mental Health \& Behavioral Disorders

Respiratory

Drug \& Alcohol Abuse

Asthma

Well Newborn

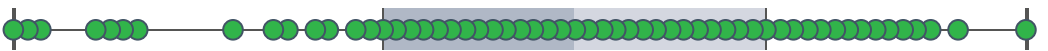

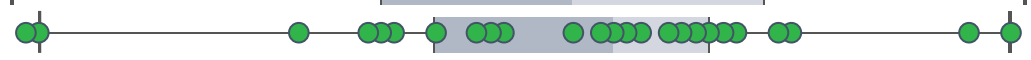

- \$000000050000000000000000 005000-00-0.

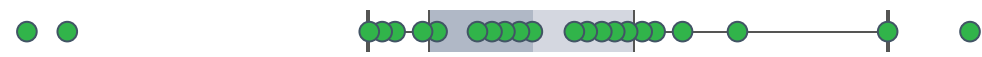

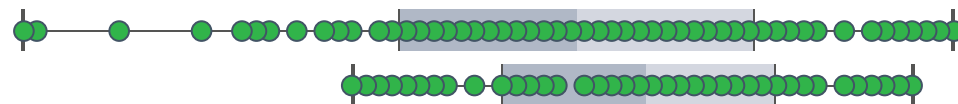

Hypertension

Cancer

Depression

GI Conditions

Pain

ADHD

Dermatological Conditions

Hematology

Genitourinary

Alzheimer's Disease

Kidney Disease

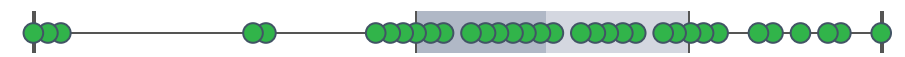

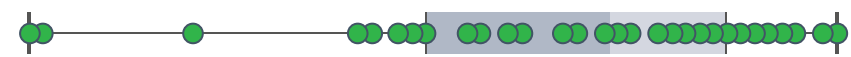

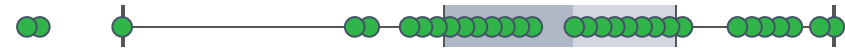

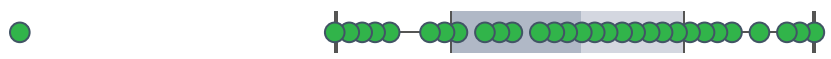

- $\quad$ \$0 $0000000000-\phi$

\$ $\quad 00000000000000000$

$\circ$

$\phi \phi \infty \phi_{0} \infty \phi$

$\phi \quad 000000 \%$ -

$\circ$

○

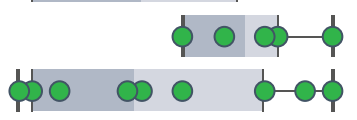

$50 \quad 60 \quad 70$

AppScript Score

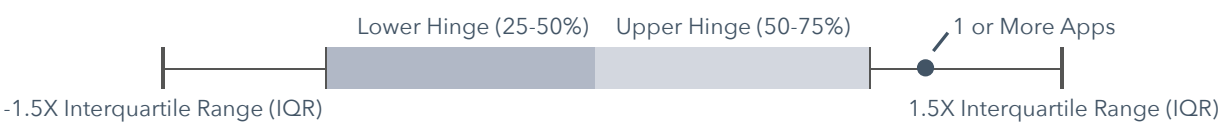




\section{Exhibit 18: Number of Published Digital Health Efficacy Studies over Time}

140

120

100

80

60

40

20

0

2007

\section{8}

TOTAL: 571 EFFICACY STUDIES BETWEEN 2007-2017 (YTD)

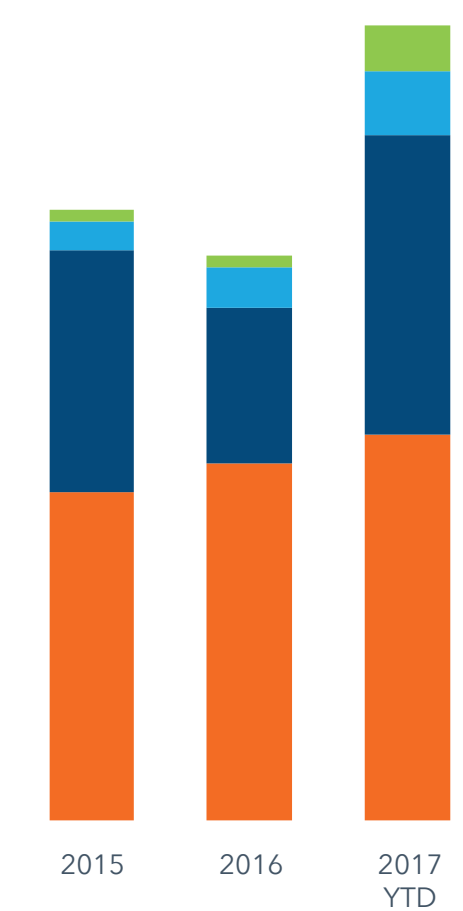

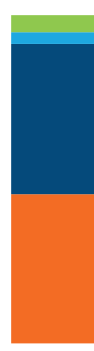

2013

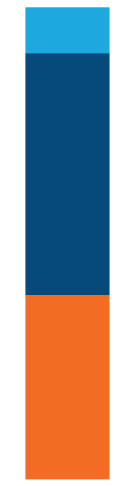

2014 the maturity of the clinical evidence - including the quantity, quality and typical results of available evidence - at the category or individual app level. For example, the American Diabetes Association has an evidence grading system in which an " $A$ " grade requires "clear" or "supporting" evidence specifically from RCTs, particularly multi-center RCTs or a meta-analysis. ${ }^{70}$

Taking this approach, a large number of Digital Health app use categories have achieved an impressive level of clinical maturity (see Exhibit 19). Three Digital Health app categories have multiple positive metaanalysis studies, including Diabetes, Depression and Anxiety. These app categories have been grouped as "Candidates for Inclusion in Clinical Guidelines" in the exhibit as the quality of evidence in these categories has begun to come into line with the typical requirements for inclusion in standard of care recommendations by guideline writers like the American Diabetes Association and the American Psychiatric Association. An additional 24 Digital Health app categories have at least one RCT and a high propensity towards positive studies that have met their primary endpoint. These categories have been grouped as "Candidates for Adoption" as individual payers and providers often make adoption decisions based on this level of clinical evidence. This grouping includes app categories with significant value creation potential including Weight Management, Asthma, COPD, Cardiac Rehabilitation and Pulmonary Rehabilitation. Categories that have not 
consistently met their endpoints (e.g., Exercise) have been grouped as "Potential Disappointments" as more nuanced approaches to applying or measuring these categories of Digital Health apps may be required to deliver more consistently positive results.
Since 2014, 11 Digital Health apps categories have changed their grouping, eight based on key studies published in 2017 alone (see Exhibit 20). Application of Digital Health apps to stroke, genitourinary conditions (e.g., incontinence), pulmonary rehab, cancer, PTSD and

\section{Exhibit 19: Maturity of Digital Health Efficacy Studies by Use Category, 2017}

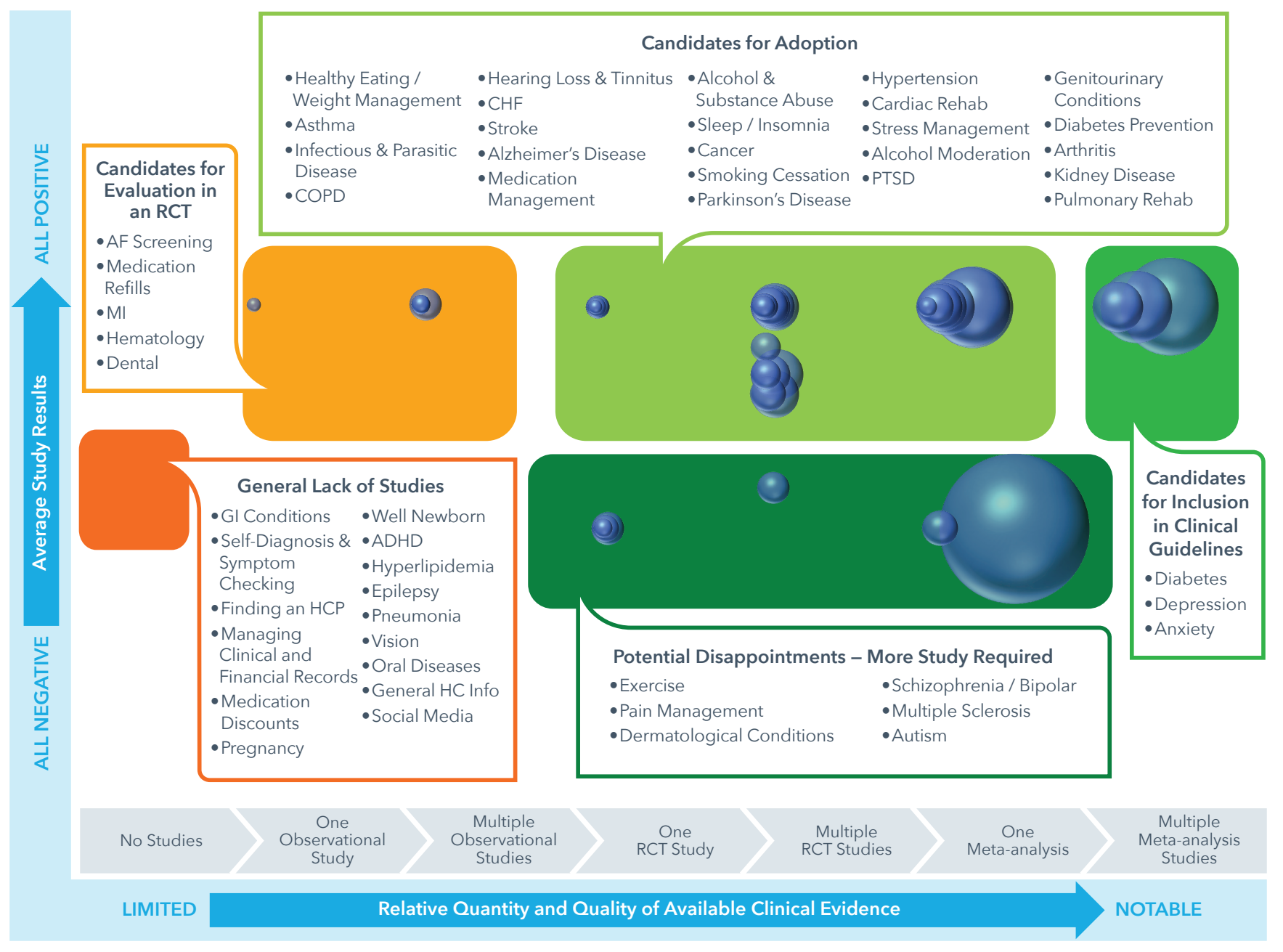

Cumulative \# of Efficacy Studies

Sources: IQVIA AppScript Clinical Evidence Database, Aug 2017

Notes: Only includes studies that evaluated the interventional value of a digital health solution (mobile or web app, connected device, or other mobile intervention such as texting) on patient outcomes such as activity levels, lab results, or healthcare resource utilization. Average of study results for the highest quality evidence available (i.e., meta-analysis > RCT > Observational). Bubble size denotes cumulative number of efficacy studies. 
arthritis were lightly studied in 2014, but now have a clinical case for adoption. Alternatively, bipolar disorder and autism were little studied in 2014, but new studies have failed to create a fresh case for adoption. The story for exercise is even more dramatic. While Exercise is one of the most studied categories, recent studies specifically the first meta-analysis in the category have called the category into question.

\section{Exhibit 20: Evidence on the Move: Significant Changes in the Maturity of Digital Health Efficacy Studies by}

\section{Category, 2014-2017}

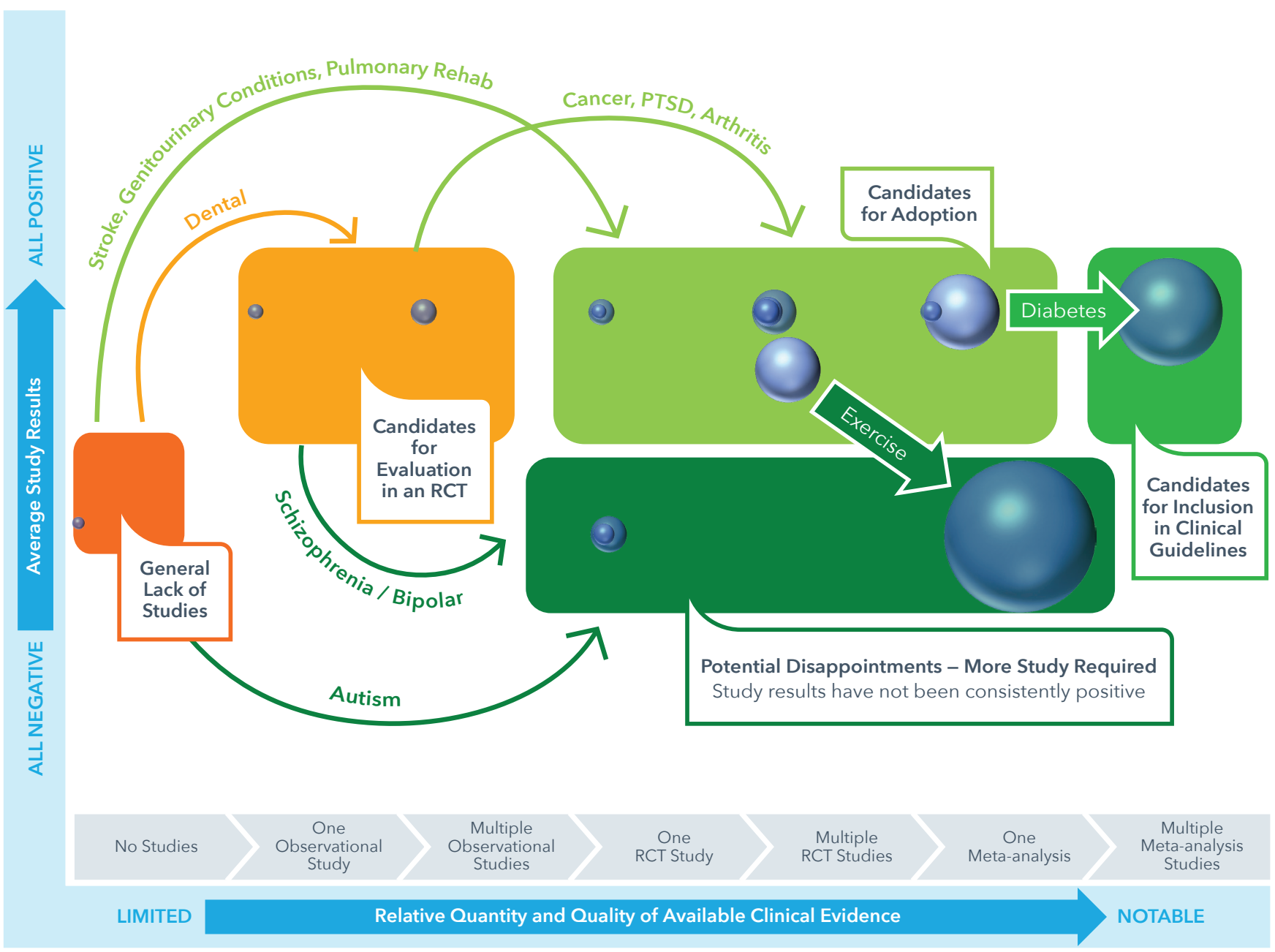

Cumulative \# of Efficacy Studies

Sources: IQVIA AppScript Clinical Evidence Database, Aug 2017

Notes: Only includes studies that evaluated the interventional value of a digital health solution (mobile or web app, connected device, or other mobile intervention such as texting) on patient outcomes such as activity levels, lab results, or healthcare resource utilization. Significant movers defined as switching category.

Average of study results for the highest quality evidence available (i.e., meta-analysis > RCT > Observational). Bubble size denotes cumulative number of efficacy studies. 


\section{Exhibit 20 Continued: Evidence on the Move: Significant Changes in the Maturity of Digital Health Efficacy Studies by Category, 2014-2017}

\begin{tabular}{|c|c|c|c|}
\hline USE & 2014 STATUS & KEY PUBLICATIONS SINCE 2014 & 2017 YTD STATUS \\
\hline Diabetes & $\begin{array}{l}\text { Candidates } \\
\text { for Adoption }\end{array}$ & $\begin{array}{l}\text { Four additional meta-analysis studies published by Hou et al (2016), Cui } \\
\text { et al (2016), Bonoto et al (2017) and Wu et al (2017) show that diabetes } \\
\text { apps have consistently delivered statistically and clinically significant } \\
\text { HbA1c improvements }(0.49 \%, 0.67 \%, 0.44 \%, 0.48 \%) \text { in Type } 2 \text { Diabetics, } \\
\text { with greater benefits in T2D than T1D, younger patients rather than older } \\
\text { patients, and patients who received healthcare professional feedback via } \\
\text { the app vs. those who did not }\end{array}$ & $\begin{array}{l}\text { Candidates } \\
\text { for Inclusion } \\
\text { in Clinical } \\
\text { Guidelines }\end{array}$ \\
\hline Stroke & $\begin{array}{l}\text { General Lack } \\
\text { of Studies }\end{array}$ & $\begin{array}{l}\text { Meta-analysis published by Liu et al (2017) showed that mobile health } \\
\text { interventions have generally mitigated key stroke risk factors including } \\
\text { glycemic control (HbA1c) and smoking cessation (abstinence) }\end{array}$ & $\begin{array}{l}\text { Candidates for } \\
\text { Adoption }\end{array}$ \\
\hline Arthritis & $\begin{array}{l}\text { Candidates } \\
\text { for Evaluation } \\
\text { in an RCT }\end{array}$ & $\begin{array}{l}\text { RCT published by Skrepnik et al (2017) demonstrated that patients with } \\
\text { knee osteoarthritis following treatment with hylan G-F } 20 \text { that used the "OA } \\
\text { GO" app increased their mobility (measured via pedometer) faster than a } \\
\text { standard follow-up control group }\end{array}$ & $\begin{array}{l}\text { Candidates for } \\
\text { Adoption }\end{array}$ \\
\hline Cancer & $\begin{array}{l}\text { Candidates } \\
\text { for Evaluation } \\
\text { in an RCT }\end{array}$ & $\begin{array}{l}\text { RCT published by Denis et al (2017) showed that the "MoovCare" web app } \\
\text { improved Overall Survival (OS) in lung cancer patients by } 7 \mathrm{mo}(58 \%) \text { vs. SoC } \\
\text { control primarily via earlier and improved initiation of optimal treatment }\end{array}$ & $\begin{array}{l}\text { Candidates for } \\
\text { Adoption }\end{array}$ \\
\hline $\begin{array}{l}\text { Pulmonary } \\
\text { Rehabilitation }\end{array}$ & $\begin{array}{l}\text { General Lack } \\
\text { of Studies }\end{array}$ & $\begin{array}{l}\text { RCT published by Bourne et al (2017) showed that "MyCOPD" app is non- } \\
\text { inferior to face-to-face pulmonary rehab }\end{array}$ & $\begin{array}{l}\text { Candidates for } \\
\text { Adoption }\end{array}$ \\
\hline PTSD & $\begin{array}{l}\text { Candidates } \\
\text { for Evaluation } \\
\text { in an RCT }\end{array}$ & $\begin{array}{l}\text { RCT published by Kuhn et al (2017) showed that PTSD patients "PTSD } \\
\text { Coach" significantly improved PTSD symptoms relative to a waitlist } \\
\text { condition control }\end{array}$ & $\begin{array}{l}\text { Candidates for } \\
\text { Adoption }\end{array}$ \\
\hline $\begin{array}{l}\text { Genitourinary } \\
\text { Conditions }\end{array}$ & $\begin{array}{l}\text { General Lack } \\
\text { of Studies }\end{array}$ & $\begin{array}{l}\text { RCTs published by Hoffman et al (2017) demonstrated the value of the Tät }{ }^{\circledR} \\
\text { app in improving urinary incontinence symptoms in women }\end{array}$ & $\begin{array}{l}\text { Candidates for } \\
\text { Adoption }\end{array}$ \\
\hline Dental & $\begin{array}{l}\text { General Lack } \\
\text { of Studies }\end{array}$ & $\begin{array}{l}\text { Observational study published by AIKlayb et al (2017) showed that the } \\
\text { iTeethey }{ }^{\top \mathrm{M}} \text { app improved mothers' knowledge of oral hygiene }\end{array}$ & $\begin{array}{l}\text { Candidates for } \\
\text { Evaluation in an } \\
\text { RCT }\end{array}$ \\
\hline Exercise & $\begin{array}{l}\text { Candidates } \\
\text { for Adoption }\end{array}$ & $\begin{array}{l}\text { Meta-analysis published by Mateo et al (2015) showed that the body of } \\
\text { RCTs in which a mobile phone app intervention was used to promote } \\
\text { weight-related health measures or physical activity showed weight loss } \\
\text { benefits but no physical activity improvements }\end{array}$ & $\begin{array}{l}\text { Potential } \\
\text { Disappointments }\end{array}$ \\
\hline Autism & $\begin{array}{l}\text { General Lack } \\
\text { of Studies }\end{array}$ & $\begin{array}{l}\text { RCT published by Whitehouse et al (2017) suggests that the Therapy } \\
\text { Outcomes By You (TOBY) app has mixed results in improving Autism } \\
\text { Treatment Evaluation Checklist }\end{array}$ & $\begin{array}{l}\text { Potential } \\
\text { Disappointments }\end{array}$ \\
\hline $\begin{array}{l}\text { Schizophrenia / } \\
\text { Bipolar }\end{array}$ & $\begin{array}{l}\text { Candidates } \\
\text { for Evaluation } \\
\text { in an RCT }\end{array}$ & $\begin{array}{l}\text { RCT published by Faurholt-Jepsen et al (2015) showed that an app for self- } \\
\text { monitoring and clinical feedback from a physician did not deliver statistically } \\
\text { significant benefits on depression or mania (Hamilton Depression Rating } \\
\text { Scale and Young Mania Rating Scale, respectively) vs the control group }\end{array}$ & $\begin{array}{l}\text { Potential } \\
\text { Disappointments }\end{array}$ \\
\hline
\end{tabular}

Sources: IQVIA AppScript Clinical Evidence Database, Aug 2017 


\section{TOP APPS}

To generate a "Top Apps" list, a top rated free and publicly available app as well as a top clinical rating app (regardless of business model) was selected across 15 high priority Digital Health app categories with high app demand and app quality. This yielded 25 Top Apps within top "Free and Publicly Available" apps and top "Clinical Rating" app classifications (see Exhibit 21). This number is less than the 30 that might be expected because certain Digital Health app categories did not have any high-quality free and publicly available apps (e.g., diabetes prevention, atrial fibrillation screening, cardiac rehabilitation, pulmonary rehabilitation) or had a free and publicly available app that also happened to be the top clinical rating app (e.g., the Walgreens app in the prescription refills category).

Certain characteristics of the Top Apps are descriptive of the current state of the art in Digital Health. The vast majority of Top Apps have at least one positive observational study demonstrating clinical efficacy (20/25; 80\%). The majority of Top Apps are iOS apps (17/25; 68\%); however, most of these apps have Android versions with similar features, endorsements and clinical evidence. More than half (14/25; 56\%) connect to an external sensor directly or via a hub such as Apple HealthKit. About one quarter (7/25; $28 \%$ - including about half of the Top Clinical Rating apps - are not publicly available to patients and

\section{Exhibit 21: Top Rated Apps}

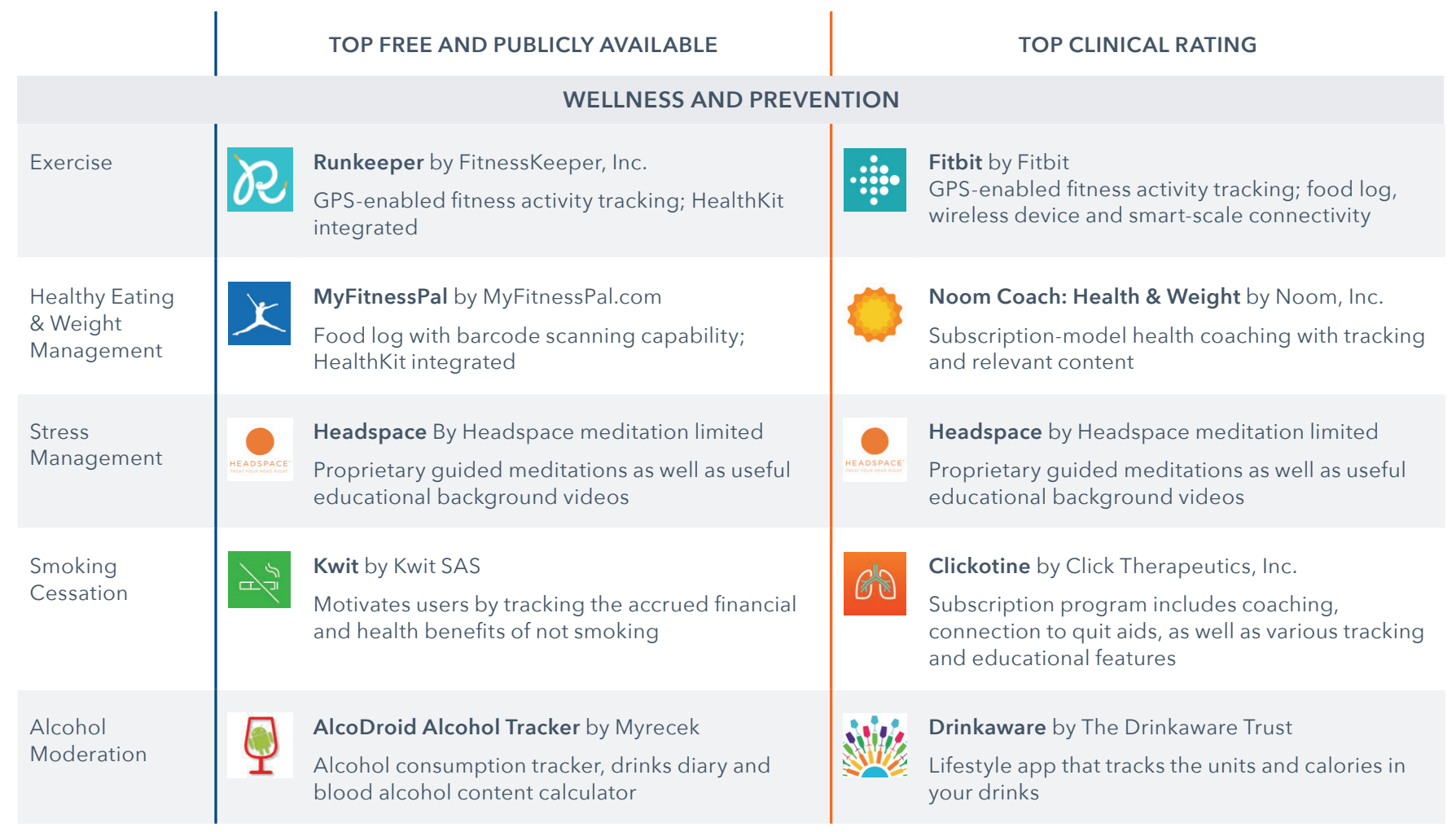




\section{Exhibit 21 Continued: Top Rated Apps}

\begin{tabular}{|c|c|c|c|}
\hline & TOP FREE AND PUBLICLY AVAILABLE & & TOP CLINICAL RATING \\
\hline \multicolumn{4}{|c|}{ CONDITION MANAGEMENT } \\
\hline $\begin{array}{l}\text { Alcohol \& } \\
\text { Substance Abuse }\end{array}$ & $\begin{array}{l}\text { My Spiritual Toolkit - AA } 12 \text { Steps App Alcoholics } \\
\text { by LOOK BEFORE YOU LEAP NET, LLC } \\
\text { Content, personal diary, and calculators for } \\
\text { recovering alcoholics }\end{array}$ & & $\begin{array}{l}\text { Pear reSET by Pear Therapeutics Inc. } \\
\text { Contains a series of interactive therapy lessons } \\
\text { with information to help support your recovery }\end{array}$ \\
\hline $\begin{array}{l}\text { Diabetes } \\
\text { Prevention }\end{array}$ & & & $\begin{array}{l}\text { Omada by Omada Health, Inc. } \\
\text { Intensive behavioral counseling (IBC) app shown } \\
\text { to promote weight loss and reduce T2D incidence }\end{array}$ \\
\hline Diabetes & $\begin{array}{l}\text { mySugr by mySugr GmbH } \\
\text { Auto-logs data via connected devices for blood } \\
\text { glucose and activity tracking. Gamification of } \\
\text { blood sugar control }\end{array}$ & & $\begin{array}{l}\text { BlueStar Diabetes by WellDoc, Inc. } \\
\text { FDA-cleared app with } 2 \text { RCTs demonstrating } \\
\text { efficacy }\end{array}$ \\
\hline $\begin{array}{l}\text { AF Screening \& } \\
\text { Dysrhythmias }\end{array}$ & & & $\begin{array}{l}\text { Kardia by AliveCor, Inc. } \\
\text { Works with FDA-cleared Kardia Mobile-a clinically } \\
\text { validated mobile EKG solution }\end{array}$ \\
\hline Hypertension & $\begin{array}{l}\text { SmartBP by Evolve Medical Systems, LLC } \\
\text { Manages blood pressure measurements and } \\
\text { track progress }\end{array}$ & & $\begin{array}{l}\text { Twine - Collaborative Care by Twine Health, Inc. } \\
\text { A collaborative care platform, designed to engage } \\
\text { patients in all care team activities }\end{array}$ \\
\hline Cardiac Rehab & & & $\begin{array}{l}\text { Healarium (Mayo Clinic Instance) by Apollo } \\
\text { Medical Holdings } \\
\text { Configurable health management app that can be } \\
\text { loaded with content for specific conditions and } \\
\text { individual patients }\end{array}$ \\
\hline Cancer & $\begin{array}{l}\text { OWise Breast Cancer by Px HealthCare B.V. } \\
\text { Helps individuals regain control during chaotic } \\
\text { times of illness and treatment }\end{array}$ & & $\begin{array}{l}\text { MoovCare by Sivan Innovation } \\
\text { Application that delivers surveys to cancer } \\
\text { patients enabling targeted follow up }\end{array}$ \\
\hline Asthma & $\begin{array}{l}\text { AsthmaMD by Mobile Breeze } \\
\text { Asthma management application. Asthma diary } \\
\text { and visualize asthma activity on a color graph. }\end{array}$ & & $\begin{array}{l}\text { Propeller Health by Reciprocal Labs } \\
\text { FDA-cleared, CE marked technology that works } \\
\text { with existing asthma and COPD inhalers }\end{array}$ \\
\hline \multicolumn{4}{|c|}{ PRESCRIPTION FILLING AND COMPLIANCE } \\
\hline $\begin{array}{l}\text { Medication } \\
\text { Refills }\end{array}$ & $\begin{array}{l}\text { Walgreens by Walgreen Co. } \\
\text { Online pharmacy serving needs for prescriptions, } \\
\text { health \& wellness products and health information }\end{array}$ & & $\begin{array}{l}\text { Walgreens by Walgreen Co. } \\
\text { Online pharmacy serving needs for prescriptions, } \\
\text { health \& wellness products and health information }\end{array}$ \\
\hline $\begin{array}{l}\text { Medication } \\
\text { Management }\end{array}$ & $\begin{array}{l}\text { Medisafe Meds \& Pill Reminder by MediSafe Inc. } \\
\text { Personalized medication management including } \\
\text { reminders, educational content, and biometrics }\end{array}$ & & $\begin{array}{l}\text { AiCure by AiCure, LLC } \\
\text { Uses patented artificial intelligence (AI) on } \\
\text { mobile devices to confirm medication ingestion. } \\
\text { It reminds and monitors if you have not taken } \\
\text { your medication with interactive visual and audio } \\
\text { guidance that automatically adjust to your needs. }\end{array}$ \\
\hline
\end{tabular}


instead require a payer or provider organization to contract with the developer. One fifth $(5 / 25 ; 20 \%)$ are FDA-cleared (MySugr, Kardia by AliveCor, Bluestar Diabetes by WellDoc, Propeller Health and reSET by Pear Therapeutics), but more (e.g., MoovCare by Sivan Innovation) may seek FDA clearance in the near future. A minority $(3 / 25 ; 12 \%)$ have a proprietary device required to use the app (Fitbit, Kardia by AliveCor and Propeller Health). No Top Apps were originally developed by pharma. One of the 25 (MySugr) is currently owned by a pharma (Roche); however, Propeller Health and BlueStar Diabetes have announced substantive partnerships with specific pharma companies and others including Medisafe have product offerings for pharma partners.

These observations point to an increasingly clinically validated and sophisticated set of apps, but also incredible diversity in terms of business models that is likely to create significant procurement challenges for healthcare providers and payers for the foreseeable future.

\section{OVERALL VALUE TO THE HEALTHCARE SYSTEM}

Guideline writers and individual healthcare institutions may make adoption decisions category-by-category or app-by-app. However, key policy setters in healthcare (e.g., the U.S. Congress) may be more interested in the overall value of Digital Health apps to the healthcare system. Major U.S. healthcare policy initiatives focused on technology adoption are rare. When such initiatives have taken place, such as the HITECH Act (which incentivized EMR adoption) and the Pathway for Biosimilars Act (which created a biosimilars approval pathway), it has generally been in an environment where persuasive cost savings arguments have been available. If policy setters were persuaded that Digital Health apps had significant cost savings potential, the Digital Health industry may see additional receptivity for policy changes in favor of Digital Health adoption such as the optimization of regulation or the creation of financial incentives. Given the acceleration in clinical evidence development in recent years, persuasive cost savings arguments surrounding Digital Health apps may be within reach.

One very direct approach that can be used to estimate the total potential cost savings of Digital Health apps in the United States is to take the subset of studies that have measured a quantitative impact on acute care utilization (e.g., emergency department visits and hospitalizations) and extrapolate those findings to appropriate national target populations to estimate associated cost savings. The advantage of this "direct acute care savings" approach is that acute care utilization is proportionately linked to cost. One only needs: (1) estimates of the number of emergency department visits and hospitalizations avoided, and (2) estimates of the typical costs of these acute care events to estimate aggregate cost savings. The disadvantage of this approach is that it fails to capture the vast majority of health outcome benefits reported in the clinical literature that have a more complicated relationship with costs such as weight loss, HbA1c moderation (in diabetics), Asthma Control Test moderation (in asthmatics), various symptom scale improvements in psychological conditions (e.g., depression), and enhanced medication adherence. The approach also fails to capture life extension which could reasonably follow from many of the health benefits identified. Finally, given that the studies that have found acute care utilization findings have been small and often observational (as opposed to randomized), results of the direct acute care savings approach will have to be updated regularly as new evidence becomes available. To summarize, the "direct acute care savings" approach is direct, but narrow, meaning that it will likely lead to straightforward, conservative estimates of potential cost savings that will require regular updates as new evidence becomes available.

The direct acute care savings approach was used to estimate total cost savings. A search for clinical evidence that had an acute care utilization finding (e.g., hospitalization and emergency department visits) 
was executed. Appropriate studies were found in five high priority Digital Health app categories including (1) Diabetes Prevention, (2) Diabetes, (3), Asthma, (4) Pulmonary Rehabilitation, (5) Cardiac Rehabilitation (see Exhibit 22 and referenced studies in the Methodology section). For each of these categories, the underlying clinical findings were used to generate a health economic model in which the intervention (and its value) were applied to an appropriate patient population. For example, since the key Diabetes Prevention study focused on seniors (at least 65 years old), the health economic model only considered benefits in this target population. In each case, estimates were based on a $100 \%$ distribution rate to the relevant target population(s), meaning that clinicians or other healthcare institutions must at least recommend the underlying solution to all of their target patients. While each target patient was assumed to be offered the solution, the health economic model accounted for the fact that not all patients would engage with and benefit from the solution. Changes in emergency department (ED) and hospitalization rates were based on findings from underlying app studies. Costs of hospitalizations or ED visits were costs to the health plan and were condition-specific if available (i.e., hospitalization costs specific to a patient whose primary diagnosis is diabetes).

The resulting health economic model suggests that a highly curated set of apps that are consistently distributed to target patients in an initial sample of five target use cases could drive U.S. cost savings of approximately $\$ 7$ billion per year (see Exhibit 23). Most cost savings are driven by reduced hospital admissions and ED visits, but given that some of the underlying studies also provided cost savings for ambulatory care,

\section{Exhibit 22: Methodology Overview for Identifying Top Apps and Use Cases for Value}

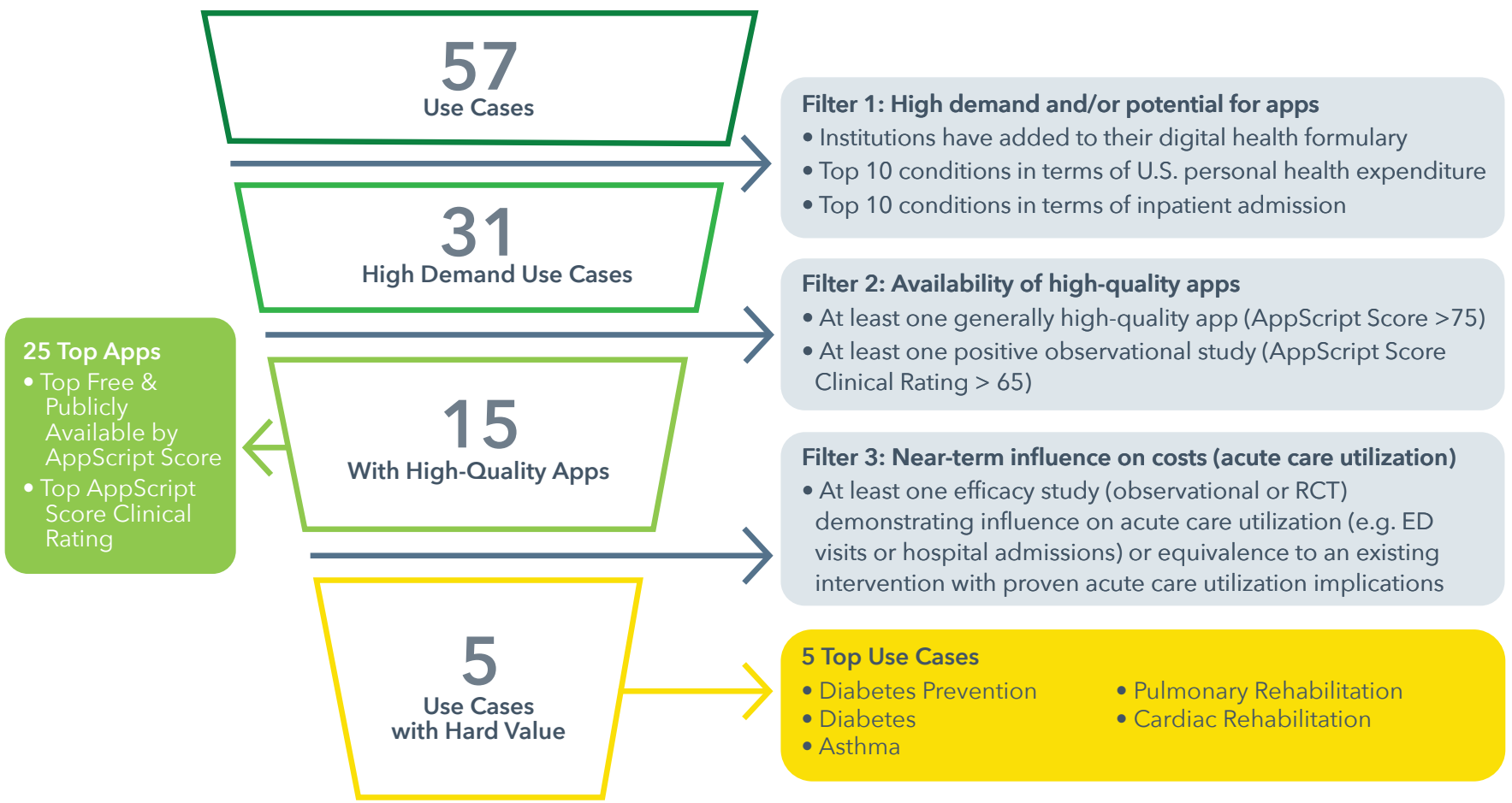




\section{Exhibit 23: Estimated Annual U.S. Cost Savings for Five Initial Uses with Potential to Reduce Acute Care Utilization, \$Bn}

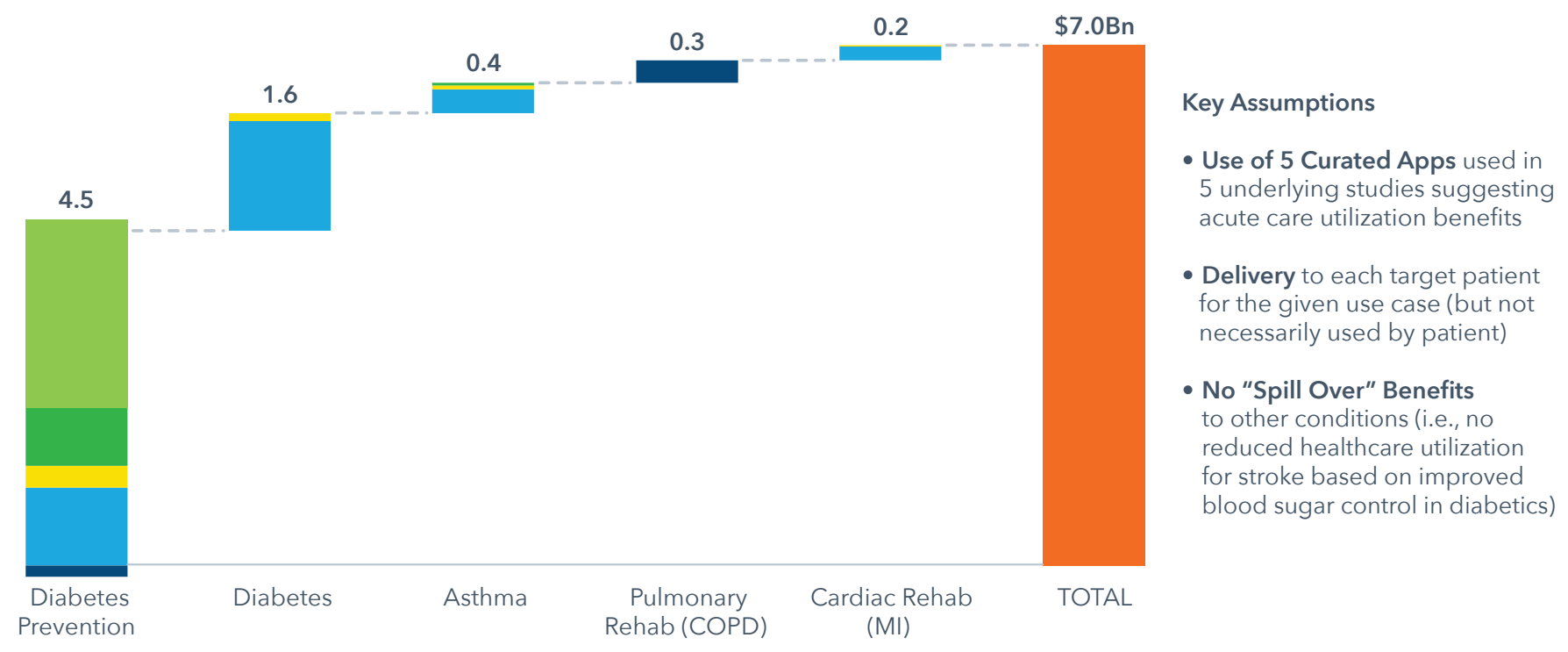

Other Intervention Costs

prescriptions and other services, these cost savings were also included when available. For example, Diabetes Prevention is somewhat of an exception in that there were significant cost savings derived from reduced physician office visits and prescriptions due to a long-term decrease in diabetes incidence. Pulmonary Rehabilitation (PR) was also an exception in that cost savings were realized due to the lower cost of appbased PR relative to face-to-face PR (an RCT simply showed app-based PR to be non-inferior to face-to-face, so no improvement in hospitalization outcomes was estimated despite the ability of PR to do so).

This level of cost-savings may be considered to be significant in light of a number of factors. First, cost savings based on a bottom-up review of individual peerreviewed publications focused exclusively on acute care utilization is a very high bar by which to measure value in healthcare. Most new medical innovations deliver higher quality care at greater cost. Digital Health apps have in some cases delivered comparable results to leading drugs (on metrics such as $\mathrm{HbA1c}$ ), albeit not in head-to-head studies. However, few drugs have shown reduced acute care utilization trends, and Digital Health apps have begun to do so. Furthermore, Digital Health apps have traditionally been priced lower than drugs and are often available for free. Digital Health apps may therefore have a more favorable cost-benefit relationship than other healthcare interventions. Second, the focus of the analysis was on acute care utilization, so the cost savings potential for ambulatory and prescription drug care was only partially assessed in this initial health economic model. Third, the model assumed no "spillover effects" meaning that no comorbid hospitalization benefits were assessed despite the fact that it would be reasonable to suggest, for example, that diabetics that better manage their blood sugar may have significantly reduced risk of myocardial infarction leading to hospitalization. Finally, these are 
only five initial use cases for Digital Health. Given that the apps saved - on average $-1.4 \%$ of total costs in their respective conditions of interest and Digital Health apps have shown clinical benefits across a broad array of conditions (see Exhibit 19), it is reasonable to expect that similar cost savings will soon become evident across an ever-increasing array of conditions. If Digital Health apps were able to save the same $1.4 \%$ of U.S. national healthcare expenditure, total U.S. cost savings potential would come to approximately $\$ 46$ billion per year (see exhibit 24). This health economic model suggests the significant cost savings potential of Digital Health with the primary caveat being that the model will have to be regularly updated as the clinical evidence base evolves.

\section{Exhibit 24: Total Potential Annual U.S. Cost Savings if Digital Health Apps Reduce Overall Healthcare Costs by Approximately $1.4 \%$ as Observed in "First 5" Use Cases, US\$Bn}

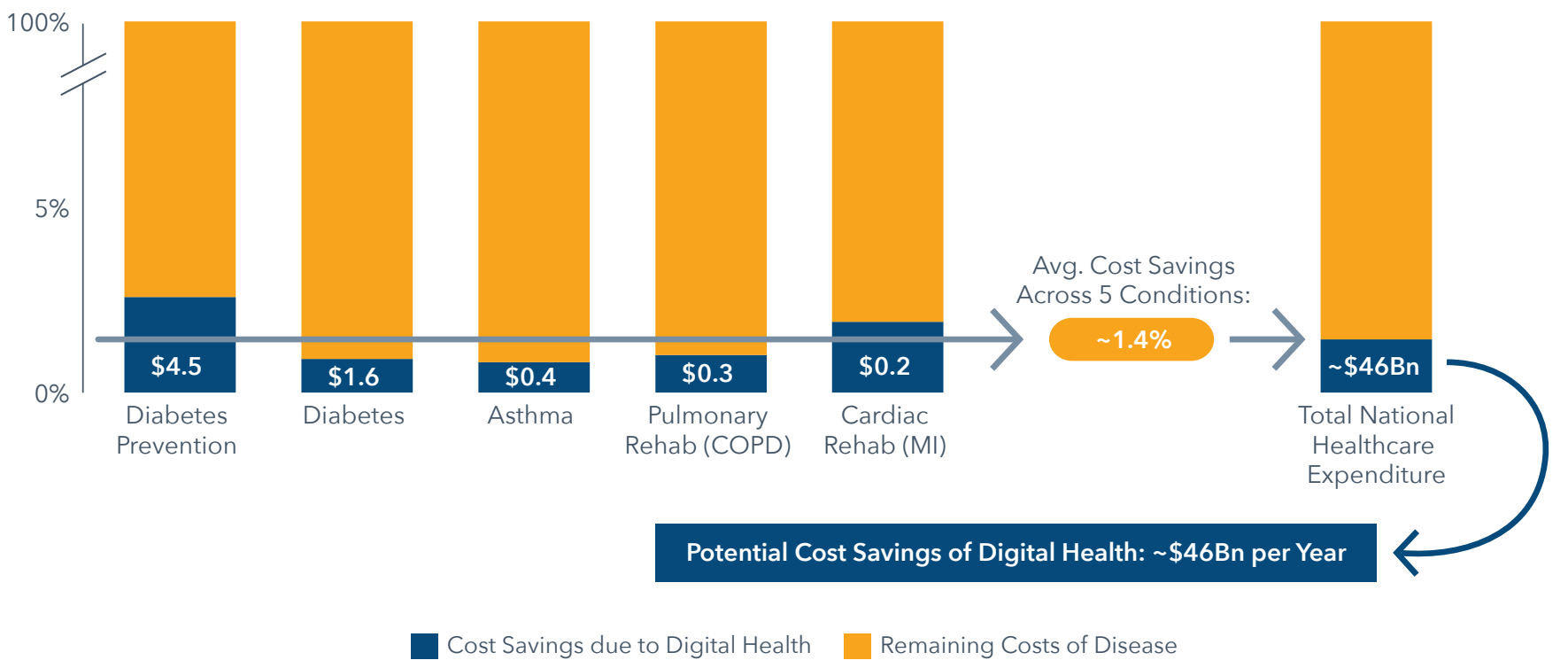

Source: IQVIA AppScript Essentials Value Model, Aug 2017

Note: Y-axis shows total case-specific health costs (100\%) and the percent mitigated by the use of Digital Health 


\section{Investments in evidence}

- Among clinical trials currently active or recruiting before Sep 2017, 869 trials worldwide now incorporate Digital Health tools, including 540 in the U.S.

- Two-thirds of these clinical studies focus on mobile app and text message interventions, which enroll over 1000 patients per study on average.

- Federal sponsorship of clinical trials has more than tripled since 2015, with two-thirds of trials run in collaboration with patient care institutions.

- Institutions focused on patient care account for $82 \%$ of all current Digital Health trials, with the top three academic and institutional sponsors being Duke University, the University of California San Francisco, and the University of Pennsylvania.

- Two-thirds of all trials incorporating Digital Health are randomized controlled trials, indicating the field has matured and the bar for evidence has risen.

\section{CURRENT TRIALS USING DIGITAL HEALTH}

As of February 2017, 869 clinical trials were being run worldwide using Digital Health technologies (see Methodology on clinical trial data), with 540 being run in the United States. The majority of these studies focus on apps and text message interventions to smartphones, however, $30 \%$ of all trials also incorporate biosensors including wearable activity monitors (see Exhibit 25). Among trials using mobile phones in the United States at least $13 \%$ use text messaging within the trial. Simple text messaging interventions have the ability to reach patients broadly while still being effective by providing reminders to perform various health behaviors, or by providing basic health information or instruction. Such simple text messaging interventions are a key focus emerging economies where patients may not have widespread internet access.
Exhibit 25: Percent of Clinical Trials Including Digital Health Technologies, By Type

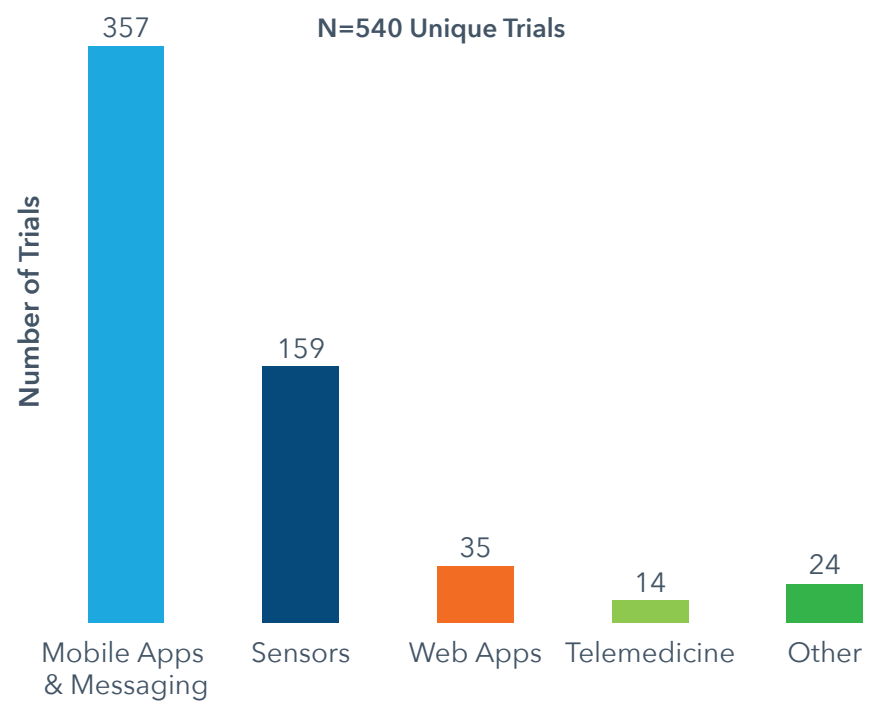

Average
Enrollment $\mathrm{N}=1156 \square \mathrm{N}=192 \square \mathrm{N}=3175 \square \mathrm{N}=99 \square \mathrm{N}=296$

Sources: Clinicaltrials.gov, Feb 2017; IQVIA Institute, Jul 2017

Notes: Total percent exceeds $100 \%$ due to inclusion of multiple digital health types in a single trial; Connected devices connect to an app, EMR or other interface; Mobile apps include use of phones to deliver text messages; Other includes offline computer apps, tablet video or virtual Interaction programs; Web apps are presumed to be mobile accessible.

Although the ability to reach broad patient populations with minimal cost is a key driver of Digital Health research, most clinical trials using these tools have moderate target enrollment (i.e., mostly pilot studies), with only 35 trials enrolling more than 1,000 patients. Enrollment into clinical trials is greatest for online web applications and mobile apps, which enroll more than 1,000 patients on average per trial, due to the relative ease of exposing large numbers of people to applications in a mobile environment, and several trials among these enrolling over 20,000 patients. Trials involving sensors, on the other hand, enrolled an average of 192 patients, demonstrating the higher burden of disseminating devices, tracking patient biometric measurements and measuring health impact. 
In total, 522,807 patients were enrolled in Digital Health trials, with trials ranging from pilots with under 20 enrollees, to those with 100,000 (see Exhibit 26). The median enrollment across Digital Health trials was 100 patients. Trials with extensive enrollments $(>10,000)$ were mostly:

- Mobile or web application trials, although one Parkinson's trial using sensors will recruit 20,00029

\section{- Rolled out to participants in a pre-built}

infrastructure, such as healthcare clinics, hospital departments, existing patients or school districts; recruiting individuals that have opted-in to research or wellness programs that allow them to be messaged; or recruiting from an existing app user base

- Efforts to build an infrastructure through recruitments (i.e., Crowdsourcing to Understand Pregnancy, PregSource)
- Text messaging trials examining the impact on clinical outcomes of improved communications either between care team members or to patients

In prior years, apps considered to be leaders in the field in terms of clinical evidence might have run only one observational study to examine impact on health outcomes. For instance, although Omada Health still has just an observational study showing its impact, it is considered the top Diabetes Prevention app. However, as the field has matured, there is increasing pressure to use interventional and randomized controlled trial design to prove value. Today, $67 \%$ of all Digital Health trials ( $n=359 / 534$ trials with design info available) are $\mathrm{RCTs}$, underscoring the rising bar for study results in this space, and the willingness of various stakeholders to invest in more complex RCT trials (see Exhibit 27).

\section{Exhibit 26: Patient Enrollment in Digital Health Trials}

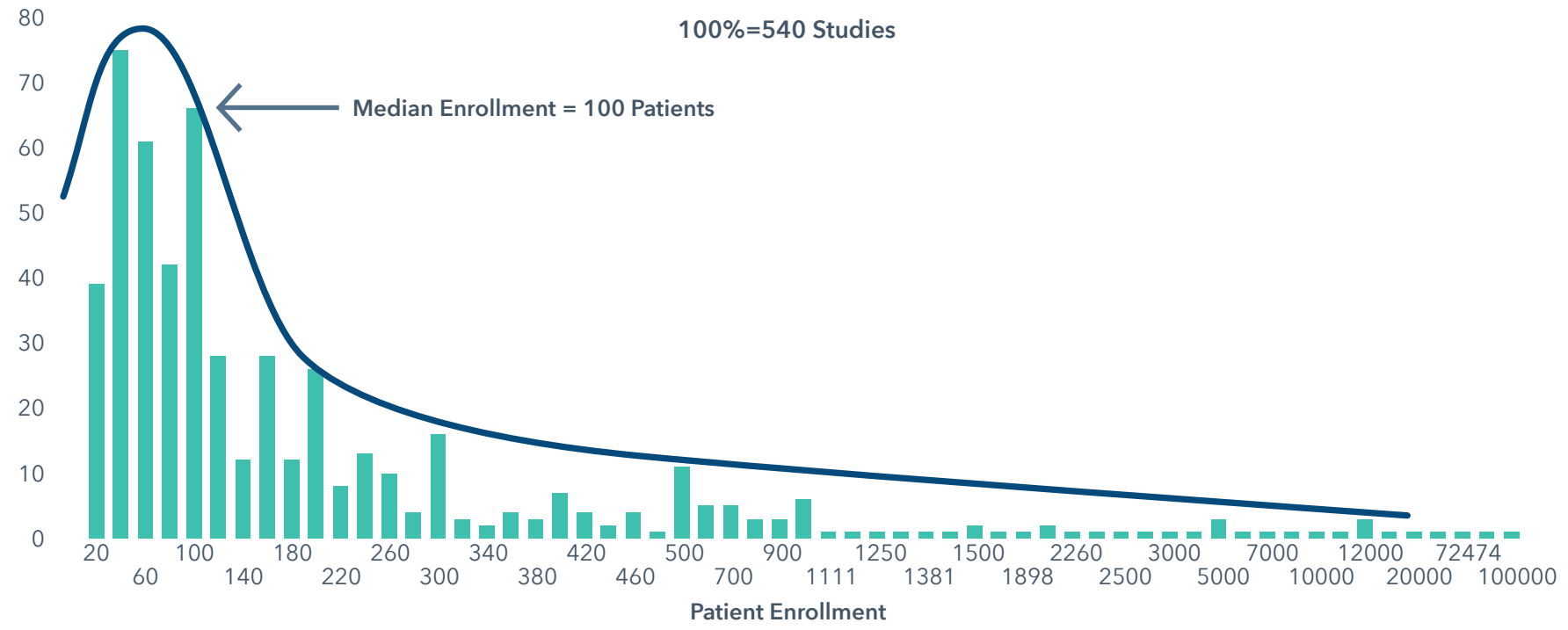

Number of Trials 
Exhibit 27: Digital Health Clinical Trial Designs

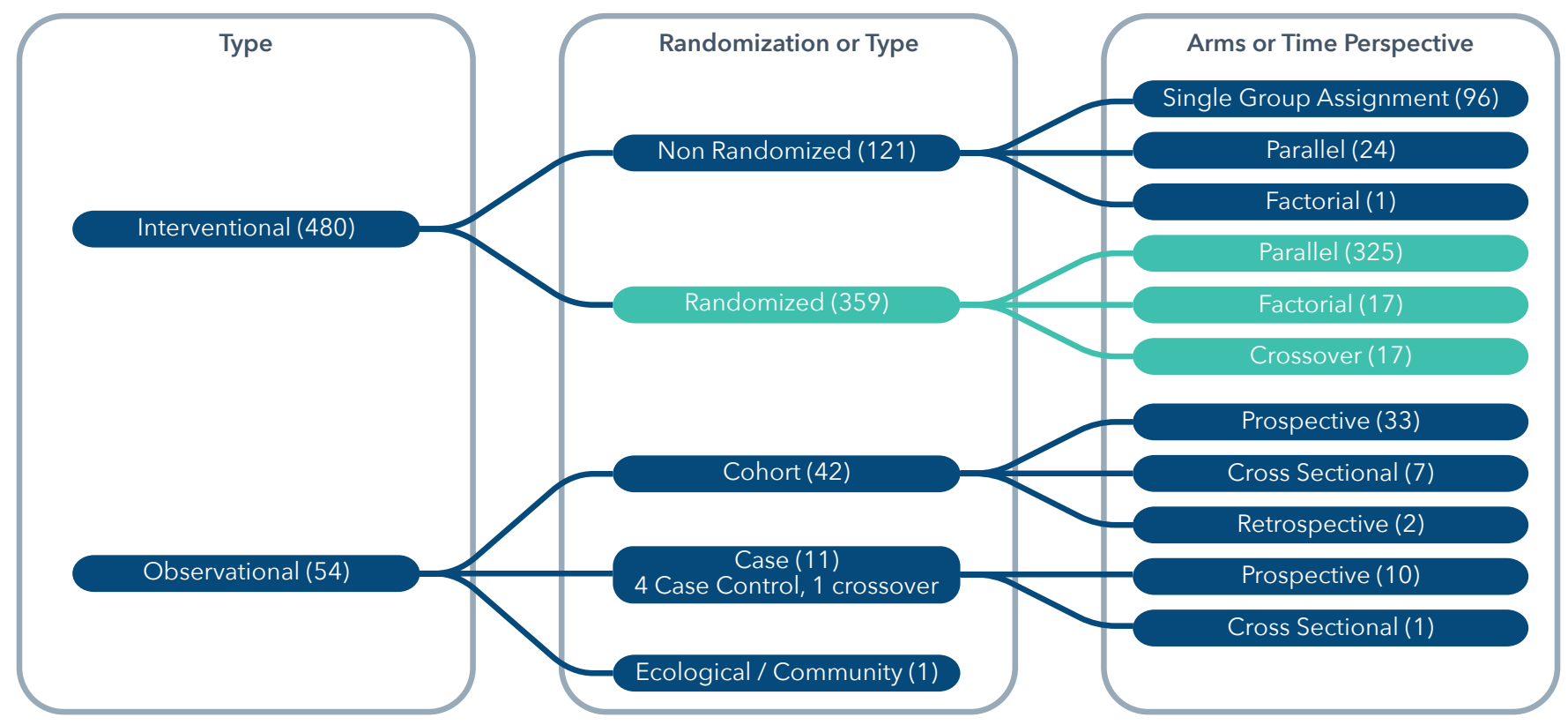

Sources: Clinicaltrials.gov, Feb 2017; IQVIA Institute, Jul 2017

Notes: Uses design designations specified on Clinicatrials.gov. Trials with the interventional model arms described as single assignment were designated as non-randomized

Among the landscape of clinical trials, very few focus specifically on the senior population where chronic disease impact could be significant. Only one clinical trial specifically focused on seniors, looking at homebased cardiac rehabilitation using sensors, although the majority of trials include both adults and seniors (see Exhibit 28). Such combined trials may provide the same long-term value to seniors in progressing treatment, since an additional three adult trials also focus on cardiac rehabilitation. A range of Digital Health interventions focus specifically on women's health issues including gynecologic diseases, pregnancy, breastfeeding, making up $10 \%$ of all trials.

Among the range of Digital Health trials, some continue to build stronger evidence for apps that already have already published, but the majority are either initial pilots to establish feasibility of new apps and connected sensors, or applications of these to healthcare to determine if they have impact on human health outcomes. Interventions are being run across use cases, with many focused on improving patient health behaviors. More than $50 \%$ of all trials are focused on treatment and prevention - areas where Digital Health tools have proven good at helping patients stick to a routine. Within treatment interventions (35\% of all trials), Digital Health tools are being tested to support patient self-management of conditions, such as stress, asthma or smoking cessation, and encourage adherence, while as supportive care tools (15\% of trials) they are being tried to impact disease symptoms and patient quality of life, including the mental health impact of disease and pain. Prevention trials often focus on digital tools for education and use in wellness programs. An additional $10 \%$ of trials focused on health services research include both patient and provider-focused applications and test the utility of new mobile health models on patient care and counseling or care coordination. 


\section{Exhibit 28: Characteristics of Patients Enrolled in Digital Health Clinical Trials}

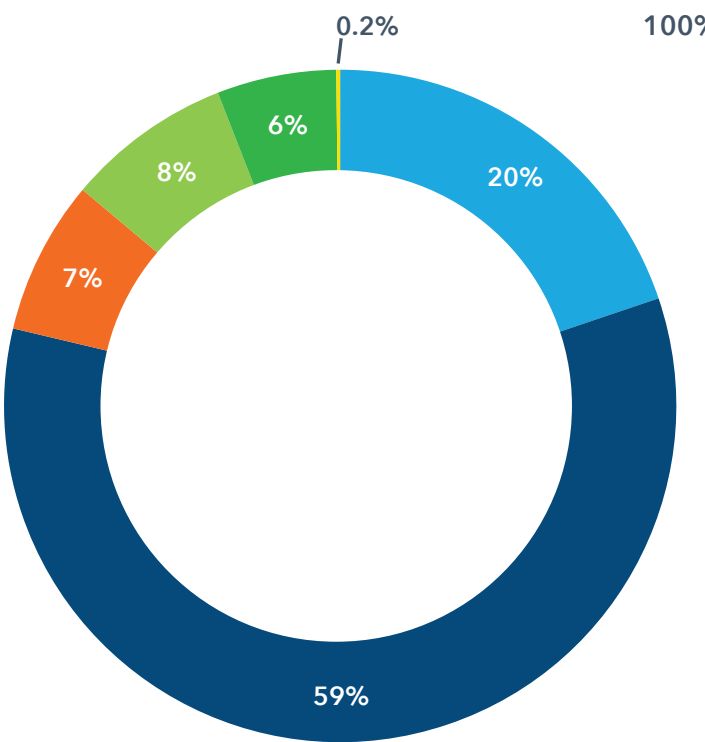

$00 \%=540$ Studies
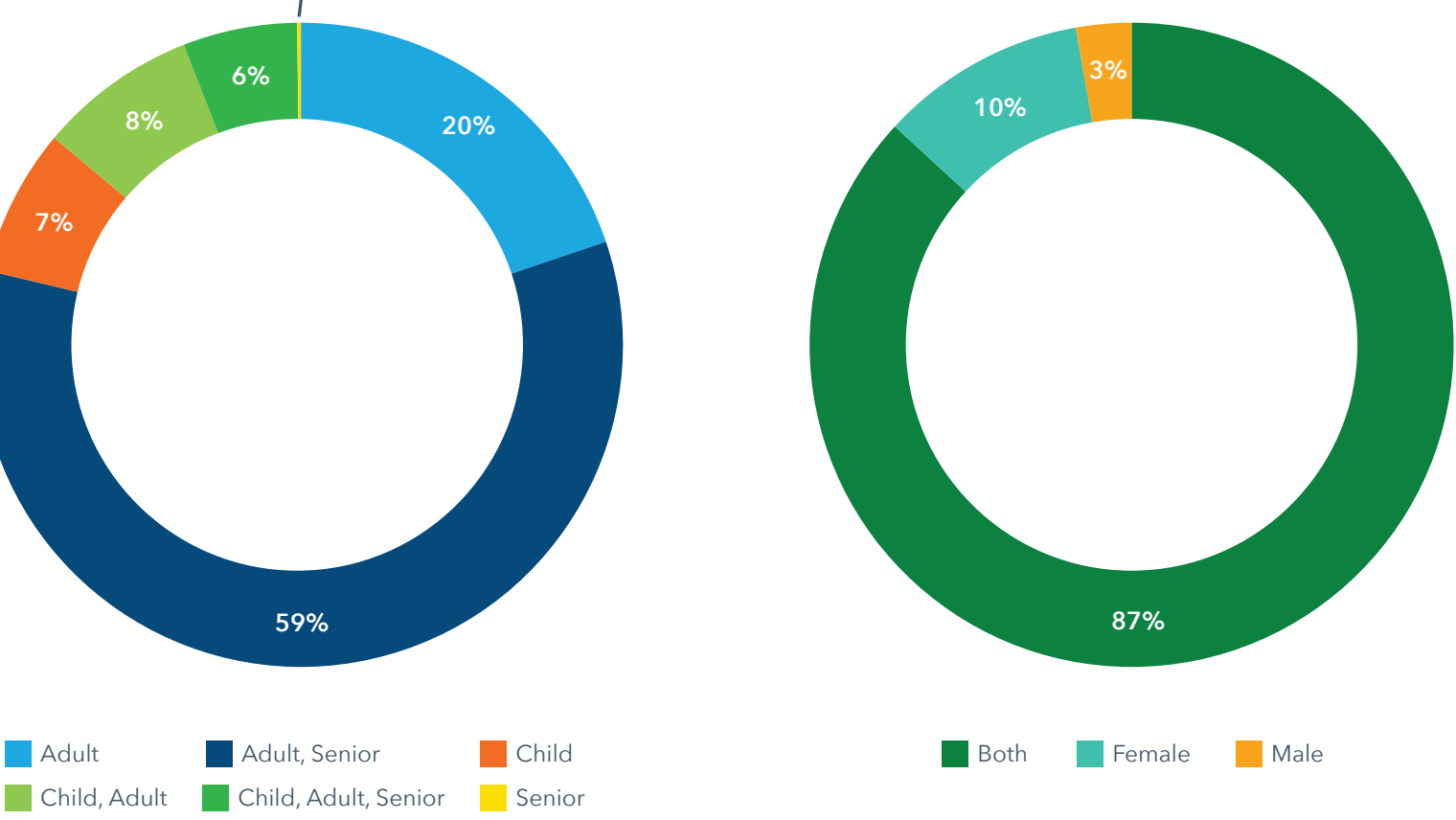

Sources: Clinicaltrials.gov, Feb 2017; IOVIA Institute, Jul 2017

The use of connected sensors and existing phone hardware for diagnosis and screening purposes still remains an emerging area of innovation, with new tools being tested for early detection of exacerbations of disease, wound and skin lesion surveillance, as well as diagnosis, such as using connected HIV testing kits that automatically trigger physician follow up.

\section{SPONSORSHIP OF DIGITAL HEALTH TRIALS}

Of the 540 trials underway in the U.S., $82 \%$ are sponsored by universities, hospitals, health systems and other patient care-focused institutions, which have aggressively begun to run pilot and proof of concept trials, seeing this as a possible route to reduce risk and readmissions. Federal government sponsorship of clinical trials has more than tripled since 2015 (now 23\%), showing a maturity in the Digital Health space and the ability to obtain government support for research deemed to have potential health benefit for enlisted populations and the public overall. Industry support for trials - from medicines manufacturers and software developers - has also risen by more than half (18\%), as companies begin to validate the health impact of apps and sensors, incorporate sensor data into trials as secondary endpoints, or use mobile devices to collect patient recorded outcomes.

Among the 95 trials sponsored by industry, the following companies have sponsored several trials each:

- Medtronic: Trials with their closed loop "artificial pancreas" sensor and app system called Harmony

- Roche: Use of a smartphone and smartwatch for digital monitoring of Multiple Sclerosis including compliance and satisfaction measurements and possibly digital biomarkers, ${ }^{71}$ use of smartphones and the ACCU-CHEK Connect system application for patient self-monitoring blood glucose (SMBG) to 
determine impact on diabetes-related distress using questionnaires; ${ }^{72,73}$ incorporation of smartphonebased Likert Scale Assessment for schizophrenia into a Phase Ilb trial in Schizophrenia ${ }^{74}$

- Posit Science Corporation (a company providing brain fitness software and services): Trials to evaluate the acceptability, feasibility and efficacy of the Moodify app as a cognitive emotional treatment for patients with major depressive disorder by measuring changes in various mood, depression and anxiety scales; $;$,76 trial using a "mobile cognitive tracking and training tool" as a device-based adjunctive treatment to "correct" disrupted neural circuitry in Parkinson's disease and impact depressive symptoms and quality of life, ${ }^{77}$ testing a home-based, online, mindfulness and adaptive cognitive training software program for soldiers and veterans with traumatic brain injury ${ }^{78}$

- Proteus Digital Health: Trials using an ingestion sensor and a wearable sensor patch to collect information about patients taking antiretroviral medications and tuberculosis medications with feedback of adherence info to healthcare providers $^{79,80}$

Leading academic institutions, which are typically aligned with large health systems, have most aggressively taken steps into the Digital Health space. The top academic sponsors of Digital Health trials in the time period examined are Duke University (32 trials), the University of California San Francisco (25 trials), and the University of Pennsylvania (19 trials). These academic universities are typically running trials across many parts of their institution and in combination with other stakeholders, as well as across conditions and use categories. For instance, studies at Duke aim to provide enhanced outpatient treatment, improve patient physician communication, improve patient self-care, test new treatment modalities and reach underserved populations (see Exhibit 29).
"In the remote patient monitoring space... [Healthcare organizations] are saying, 'We get that there could be value, but can we actually take our most expensive patients, our most at-risk patients - whether that be in terms of health or in terms of dollars - and make an impact on the way we care for them right now?' What we're seeing is that the answer to that is 'yes'. This is the first area we're seeing a real difference being made and there's a real acknowledgement of that across the industry. They are saying... 'Now, it's time to start doing this ourselves - to take this from a pilot into reality in terms of how we manage these kinds of conditions. We can lower the cost and improve the quality of care by adopting these."'

Marc Sebes, Vice President of Product Management, Validic 


\begin{tabular}{|c|c|c|c|c|c|}
\hline $\begin{array}{c}\text { DIGITAL } \\
\text { HEALTH TOOL }\end{array}$ & DISEASE & TRIAL & PARTNER & $\begin{array}{l}\text { OUTCOMES } \\
\text { TESTED }\end{array}$ & INTENT \\
\hline SMART App & $\begin{array}{l}\text { Sickle Cell } \\
\text { Disease }\end{array}$ & $\begin{array}{l}\text { Web-aided, mobile- } \\
\text { based Personal } \\
\text { Health Reporting } \\
\text { Service to enhance } \\
\text { outpatient treatment } \\
\text { after discharge from } \\
\text { an acute care setting }\end{array}$ & SickleSoft & $\begin{array}{l}\text { - Acute care utilization } \\
\text { - Adherence to hydroxyurea } \\
(\mathrm{HU}) \text { administration } \\
\text { - Adherence to post-acute } \\
\text { care out-patient follow } \\
\text { up visit }\end{array}$ & $\begin{array}{l}\text { - Increase patient } \\
\text { involvement in their } \\
\text { treatment } \\
\text { - Improve patient to } \\
\text { doctor communication } \\
\text { - Develop patient-doctor } \\
\text { relationships } \\
\text { - Decrease in readmission } \\
\text { to an acute care facility }\end{array}$ \\
\hline $\begin{array}{l}\text { Recovery Record } \\
\text { (RR) Mobile App }\end{array}$ & $\begin{array}{l}\text { Type-1 } \\
\text { Diabetes } \\
\text { (T1D) }\end{array}$ & $\begin{array}{l}\text { Pairing a mobile } \\
\text { application with } \\
\text { individual therapy } \\
\text { to help T1D patients } \\
\text { identify their } \\
\text { triggers for insulin } \\
\text { omission in their } \\
\text { natural environment } \\
\text { and cope more } \\
\text { effectively }\end{array}$ & $\begin{array}{l}\text { National Institute } \\
\text { of Diabetes and } \\
\text { Digestive and } \\
\text { Kidney Diseases } \\
\text { (NIDDK) }\end{array}$ & $\begin{array}{l}\text { - Diabetes blood tests } \\
\text { - Mobile technology } \\
\text { engagement /use } \\
\text { - Medical utilization: Primary } \\
\text { care and Emergency } \\
\text { department care visits }\end{array}$ & $\begin{array}{l}\text { - Reduce intentional } \\
\text { insulin omission for } \\
\text { weight control among } \\
\text { individuals with T1D } \\
\text { - Increasing patients' } \\
\text { capacity to effectively } \\
\text { cope with psychological } \\
\text { barriers to self-care } \\
\text { using acceptance and } \\
\text { mindfulness strategies }\end{array}$ \\
\hline $\begin{array}{l}\text { VIDA Mobile } \\
\text { Health } \\
\text { Cardiovascular } \\
\text { Prevention } \\
\text { Program }\end{array}$ & $\begin{array}{l}\text { Coronary } \\
\text { Artery } \\
\text { Disease } \\
\text { (CAD) }\end{array}$ & $\begin{array}{l}\text { App, Fitbit and } \\
\text { health coaching } \\
\text { supported } \\
\text { intervention for } \\
\text { patients who have } \\
\text { recently graduated } \\
\text { from cardiac } \\
\text { rehabilitation }\end{array}$ & Vida Health, Inc. & $\begin{array}{l}\text { - Physical activity via Fitbit } \\
\text { steps, distance traveled } \\
\text { and floors climbed } \\
\text { - Body composition } \\
\text { - Quality of life (QOL) } \\
\text { - Medication adherence } \\
\text { - Change in fitness (peak } \\
\text { VO2mL/kg/min) }\end{array}$ & $\begin{array}{l}\text { - Determine if a } \\
\text { disease management } \\
\text { intervention for CAD } \\
\text { patients can improve } \\
\text { dietary choices, physical } \\
\text { activity, medication } \\
\text { management, patient } \\
\text { understanding of health } \\
\text { status }\end{array}$ \\
\hline $\begin{array}{l}\text { NeuroSky } \\
\text { MindWave } \\
\text { Mobile EEG } \\
\text { device / Mobile } \\
\text { Neurofeedback } \\
\text { App on iPod } \\
\text { Touch }\end{array}$ & $\begin{array}{l}\text { Post } \\
\text { Traumatic } \\
\text { Stress } \\
\text { Disorder } \\
\text { (PTSD) and } \\
\text { traumatic } \\
\text { brain injury } \\
\text { (TBI) }\end{array}$ & $\begin{array}{l}\text { Pain management } \\
\text { using a mobile EEG } \\
\text { device in veterans } \\
\text { with PTSD and TBI }\end{array}$ & $\begin{array}{l}\text { National Center for } \\
\text { Complementary } \\
\text { and Integrative } \\
\text { Health }(\mathrm{NCClH})\end{array}$ & $\begin{array}{l}\text { - App adherence } \\
\text { - Self-reported pain score } \\
\text { - Effects on drug abuse, } \\
\text { violence, and suicidality }\end{array}$ & $\begin{array}{l}\text { - Portable, low-cost } \\
\text { intervention to reduce } \\
\text { the risk of opioid abuse } \\
\text { and barriers to traditional } \\
\text { mental health treatments }\end{array}$ \\
\hline $\begin{array}{l}\text { mHealth } \\
\text { Pain Coping } \\
\text { Skills Training } \\
\text { (mPCST) } \\
\text { protocol for } \\
\text { Medically } \\
\text { Underserved } \\
\text { Communities }\end{array}$ & Cancer Pain & $\begin{array}{l}\text { Intervention } \\
\text { including } \\
\text { smartphone } \\
\text { tools (e.g., text } \\
\text { messages, images, } \\
\text { and preloaded } \\
\text { intervention content) }\end{array}$ & None & $\begin{array}{l}\text { - Pain severity/disability } \\
\text { - Self-efficacy for pain } \\
\text { control } \\
\text { - Coping strategies } \\
\text { - Depressive symptoms } \\
\text { - Fatigue } \\
\text { - Health-Related QOL }\end{array}$ & $\begin{array}{l}\text { - Better aid medically } \\
\text { underserved areas } \\
\text { - Pilot a protocol designed } \\
\text { to reduce persistent } \\
\text { cancer pain in breast } \\
\text { cancer survivors and } \\
\text { disability in patients with } \\
\text { low literacy } \\
\text { - Allow intervention to } \\
\text { extend into the patients' } \\
\text { homes }\end{array}$ \\
\hline
\end{tabular}




\section{Accelerating use in medicine}

- Adoption of Digital Health by clinicians is increasing but is far from mainstream; with only $26 \%$ of clinicians currently recommending patient engagement technologies and $13 \%$ using remote patient monitoring technologies according to the AMA survey.

- For the health system to adopt Digital Health tools their use must be made safe and easy.

- Though they offer significant potential impact on patient care and healthcare costs, few condition management apps have reached the level of workflow integration necessary to gain widespread physician adoption.

- Investments by healthcare and provider organizations in Digital Health has become more mature, shifting from pilot programs to more fullscale rollouts, with an estimated $20 \%$ of large health systems moving from initial pilots to rollouts and $75 \%$ of the rest expanding pilots or adding a second.

- Workflow integration remains a key barrier. Vendors have stepped in to allow healthcare stakeholders to more easily make use of Digital Health data; enabling both across-the-board integration of a menu of digital patient engagement tools into EMRs, or the integration of data from specific apps.

- The use of digital tools at healthcare facilities are likely to move beyond pilot phase within five years, be mainstream for most organizations managing patients with specific conditions in ten years and help treat well and sick patients alike in 15 years.

\section{THE PATH TO HEALTH SYSTEM ADOPTION}

Although Digital Health apps have the potential to deliver significant value to the U.S. health system in terms of improved human health outcomes and reduced costs, the realization of this value can only occur if the health system enables the delivery to patients of specific high-quality apps. Lacking support from health system stakeholders, such as clinicians, health plans or employers, apps may not be accessible to patients nor effective. At least two of the top 25 apps identified in this report are by "prescription use only" as per the FDA and other Top Apps have opted to distribute their apps only through employers, health plans or healthcare providers. Additionally, most studies showing evidence of human health benefit involved direct distribution from providers to patients, or have shown apps to be more effective with clinician participation. To make many leading Digital Health apps affordable to critical patient populations, subsidies from other healthcare actors may also be necessary.

As outlined in our 2015 study, for the health system to adopt Digital Health more broadly, additional progress must be made in a number of areas, including the curation and evaluation of apps; the creation of best practices around privacy and security; increased stakeholder recognition of value; the establishment of regulatory guidelines and reimbursement models, and improvements in workflow integration for physicians. ${ }^{69}$ In essence, use of effective Digital Health tools must also be made safe and easy.

These areas are still a key focus for clinicians. In a recent survey of 1,300 physicians, the AMA found that clinicians have a broad array of requirements for the adoption of Digital Health including - in rough order of importance (1) coverage by malpractice insurance, (2) data privacy and security assurances from EHR vendors and their practices, (3) ease of use, including EHR workflow integration and support, (4) reimbursement for time spent and (5) proven efficacy as good or better than traditional care. ${ }^{59}$ Health plan executives, pharmacy and medical directors similarly focus on ease of use and EHR integration in addition to the clinical and economic benefits that help them meet their health plan quality metrics. ${ }^{81}$ 
"This is a rapidly moving area that it's very much in need of... physician involvement. The experience that physicians have had with their electronic health records over the last several years hasn't been particularly good. There has been a lot of disruption in terms of work and productivity, and an increasing chorus of complaints about the usability of these products and their contribution to physician burnout. Many of us feel that physicians weren't adequately included in the design of these products, or decisions about the[ir] use... and that has contributed to the issues that physicians have with them today. [Those issues] will only be amplified as we get further into the digital health space... if physicians aren't more involved in their development and their implementation."

\section{Dr. Michael Hodgkins, Vice President} and Chief Medical Information Officer, American Medical Association, August 9th, 2017
Despite taking strides in these areas, even the Top Apps identified in this publication do not completely address these key requirements along the path to adoption. Top App developers have generally invested in delivering apps with high patient usability as well as clinical studies demonstrating efficacy, yet have not consistently answered the question "How does this fit into a clinician's practice?"

Current levels of use remain low with $26 \%$ of clinicians currently recommending patient engagement technologies (generally aligned to use in wellness and prevention) and approximately $13 \%$ remote patient monitoring technologies (generally aligned to use in condition management) according to the AMA survey. The underlying requirements along the path to adoption are being met to varying degrees by even the leading apps (see Exhibit 30). ${ }^{59}$

\section{ACCELERATORS AND BARRIERS}

Although app developers have a role to play in bridging the gap between the current state of the industry and the requirements of the health system, various policy and technical infrastructure industry initiatives stand to further accelerate adoption over the next five years. Many of these accelerators are maturing rapidly and thereby are reducing barriers to the use of Digital Health.

Finding the best apps: curation platforms and Digital Pharmacy and Therapeutics Committees

A growing interest in Digital Health apps is being met by a variety of curation efforts that assist providers and health plans to identify high-quality apps. Eighty-six percent (86\%) of physicians surveyed by the AMA felt Digital Health would provide them an advantage in caring for patients and $45 \%$ were enthusiastic about remote patient monitoring. ${ }^{59}$ However, the selection of initial uses for apps - let alone the selection of individual apps that are effective and easy to use remains a daunting task, even for large health institutions, requiring research on app benefits and the alignment of clinical stakeholders for app deployment. 
Exhibit 30: Fraction of Top Apps that Adequately Address Providers' Key Requirements and Emerging Accelerators of Adoption
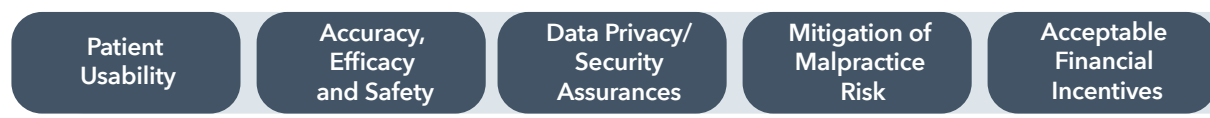
Acceptable
Clinician Workflow
\& Usability
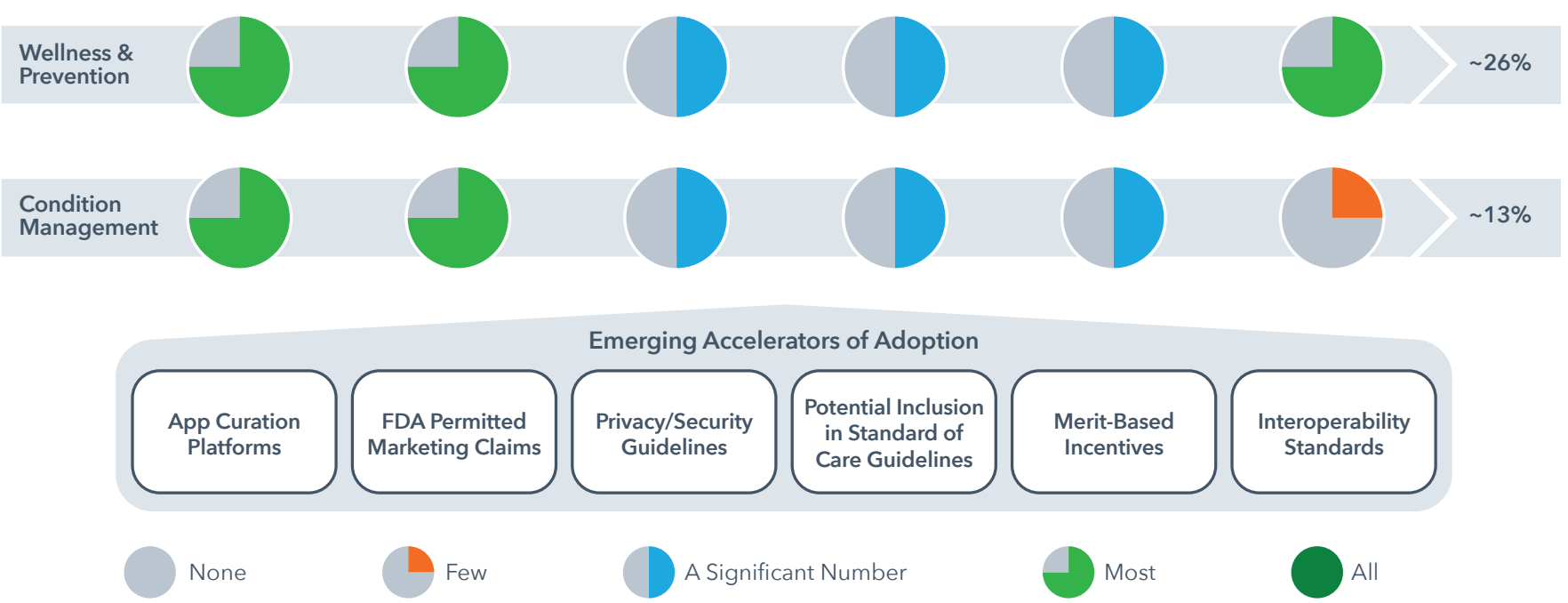

Source: American Medical Association, Digital Health Study, Sep 2016; IQVIA Institute Analysis, Sep 2017

The large number of ineffective apps are a source of noise that needs to be filtered out via appropriate curation efforts.

To aid in this selection, a large number and variety of organizations such as the app stores, digital content publishers, clinician-led organizations, app assessment and accreditation organizations and app curation and delivery platforms, have published "Top Apps" lists or have otherwise undertaken initiatives to endorse apps based on meeting specific quality criteria. A small subset of these organizations differentiate themselves by publishing app reviews based heavily on the availability of objective, peer-reviewed clinical evidence (e.g., AppScript, RankedHealth).

Health plans and providers are themselves moving towards creating curated app lists or "formularies" of apps approved for use by the institution, sometimes also making available lists of free apps for their patients to use (e.g., Cleveland Clinic Mobile Apps list). Such institutional Digital Therapeutics Formularies clarify for individual clinicians not only what apps are potentially useful, but also what apps institutional leadership approves for recommendation to appropriate patients. To accelerate the creation and management of such institution-specific formularies, app curation platforms provide a knowledgebase about available apps as well as technical acumen that empower key clinical decision makers on "Digital Pharmacy and Therapeutics ("P\&T") Committees," and the like, to make formulary decisions. Similar to how hospitals currently run P\&T Committees that leverage data on pharmaceutical efficacy, safety and pricing to select drugs for hospital formularies, app curation organizations provide similar details to support the creation of digital formularies. 
By making available reliable objective, third party data on the health value and technical aspects of apps, app curation platforms can speed adoption into Digital Therapeutics formularies, and facilitate physician prescribing. One top ten U.S. Integrated Delivery Network spent more than two years tasking their physician community with the evaluation of apps for use across their organization. When no consensus emerged after this long evaluation period, IOVIA AppScript was asked to assist with Digital Health app curation and prescription delivery using their platform. Within months, the healthcare provider had rolled out their Digital Health pilot and had prescribed apps to over one thousand patients. Overall, such curation platforms and the data they provide help defeat "analysis paralysis" so that organizations can scale the use of Digital Health tools more quickly.

Aligning on appropriate data protections: privacy and security guidelines

Initial privacy and security guidelines - and new initiatives to create such guidelines - have emerged to address stakeholder concerns in these areas. Despite the potential clinical value of Digital Health apps, many healthcare institutions and individual clinicians remain skeptical of app developers' management of patient data. A number of published studies have taken issue with the quantity and quality of Digital Health app privacy policies, with many apps failing to accurately describe circumstances in which apps share patient data with third parties. ${ }^{83,84}$ One widely reported study found that $86 \%$ of a sample of 71 popular health apps had at least two critical security vulnerabilities. ${ }^{85}$ These studies have called on healthcare professionals to consider risks to patient data when recommending Digital Health apps, but have fallen short of providing concrete guidelines or associated accreditation
"We live in a time of rapid development in the digital world...but appearing in disguise among these positive products are other digital socalled advancements that don't have an appropriate evidence base, or just don't work that well - or actually impede care, confuse patients and waste our time...From ineffective electronic health records, to an explosion of direct-to-consumer digital health products, to apps of mixed quality, this is the digital snake oil of the early 21st century... Even those digital products that might be helpful often lack a way of enriching the relationship between the physician and the patient."

James L. Madara, MD, Executive Vice President and CEO, American Medical Association 
systems that define apps with acceptable privacy and security policies in place, versus those that do not, to an audience of non-experts (e.g., clinicians).

Several institutions, including the United Kingdom's National Health Service (NHS) and the not-for-profit Xcertia, are now attempting to address the need for clarity around privacy and security (as well as other parameters like usability and operability) by providing standardized app-review guidelines that may in some cases result in accreditation by a reputable third party. The NHS has published "Digital Assessment Questions Beta" which ask app developers for key privacy and security details, which are then subject to review. Apps that provide acceptable answers become eligible to be listed on the "NHS Apps Library" (also currently in beta). Similarly, Xcertia - a collaboration between the AMA, Healthcare Information and Management Systems Society (HIMSS), American Heart Association (AHA) and DHX Group - seeks to bring a series of stakeholders together to ultimately publish guidelines (for phased release in 2018) that app developers' and those reviewing them can use to create and evaluate apps. ${ }^{86}$ The goal of this group is to enable healthcare providers to reduce the amount of time required to assess apps and speed app development time. By creating a standardized and reputable means of evaluating privacy and security, such guidelines may be helpful in creating more trust on behalf of clinicians, and ultimately more rapid adoption.

Endorsing clinical value: the FDA and standard of care guidelines

Although the availability of peer-reviewed efficacy studies demonstrating the quantitative interventional value of Digital Health apps has grown, to have a sizeable impact on adoption, clinical evidence often needs to be summarized and ultimately endorsed by credible third parties, since few individual institutions, let alone individual clinicians, can keep up with the rapid advances underway. Routes to endorsement of app efficacy are now emerging via FDA approval
"When put to the test, the majority of mobile health apps failed security tests and could easily be hacked. Such vulnerabilities could allow the apps to be tampered and reverse-engineered, put sensitive health information in the wrong hands and, even worse, potentially force critical health apps to malfunction. Surprisingly, US Food and Drug Administration (FDA)-approved apps and formerly UK National Health Service (NHS)-approved apps were among the vulnerable mobile health apps tested, indicating that there is more work to be done by governing bodies to better understand the cybersecurity threats to mobile apps and improve the minimum acceptable security standards or regulations for mobile app development." 82

Arxan. 5th Annual State of Mobile Application Security Report: Perception vs. Reality. January 2016 
pathways awarding permitted marketing claims, and as this report has argued - potential inclusion in clinical standard of care guidelines of certain categories of Digital Health apps.

Both clinical standard of care guidelines and FDA permitted marketing claims can have a dramatic influence on health plan coverage decisions and medical malpractice - both of which are key barriers to adoption of Digital Health tools by clinicians. Health plans generally provide favorable coverage of treatments that are highly recommended by clinical guidelines and often provide at least some coverage of treatments that are found safe and effective by the FDA. The concept of "medical standard of care" is also central to the field of medical malpractice, where negligence (and legal liability) is generally a function of whether

\section{"We're always surprised with} the amount of payer interest there is as payers try and grapple with reaching their star ratings and grapple with population health in general. A lot of payers and providers are starting to open up a bit more and be more activated in how to take more proactive care of their patient population due to changing incentives."

Christine Lemke, President, Evidation Health or not a clinician's conduct or recommendations have strayed significantly from the typical expectations of the profession. By endorsing particular clinical evidence, clinical guidelines and FDA permitted marketing claims simultaneously address the health system's concerns around clinical value, medical malpractice and incentives.

To date, clinical guidelines writers and the FDA have had a muted role in endorsing the available clinical evidence surrounding Digital Health apps; however, this is beginning to change. At the time of publication, no known standard of care guidelines have endorsed Digital Health apps, but particularly the diabetes area, which has seen the publication of many RCTs and metaanalysis studies that have consistently found statistically significant HbA1c and other clinical improvements (for more detail, see Consideration for Inclusion in Diabetes Guidelines), may be eligible for inclusion.

Digital Health apps are also now flowing through regulatory pathways that endorse their efficacy by awarding efficacy claims. While the FDA has cleared dozens of mobile medical devices and wearable sensors for use by patients, these clearances have generally been via the " $510(k)$ process" through which products are cleared for market based on demonstration of substantial equivalence to a predicate device. Most $510(k) s$ do not require prospective clinical study data to support clearance, and therefore do not benefit from any endorsement of clinical data - however tacit - by the FDA. This mobile medical app clearance paradigm may have changed in September 2017, when the FDA announced that Pear Therapeutics could market its reSET mobile medical app device system as a "prescription-only adjunct treatment" for use in Substance Use Disorder (SUD). The app was approved via the "de novo" request premarket pathway - a market clearance pathway for some low- to moderate-risk devices that are novel and for which there is no legally marketed predicate device to which the device can claim substantial equivalence. 
To date, it is unclear why clinical guidelines have not yet emerged for the use of Digital Health in diabetes. The American Diabetes Association has an evidence grading system in which an " $A$ " grade requires "clear" or "supporting" evidence specifically from a multi-center RCT or a meta-analysis study that incorporates an evidence quality rating system. ${ }^{89}$ Digital Health apps within the diabetes category have seen the publication of many RCTs and meta-analysis studies that have consistently found statistically significant $\mathrm{HbA} 1 \mathrm{c}$ and other clinical improvements and few safety concerns. For example, one meta-analysis study featuring an evidence quality rating system explored the influence of mobile phone applications on glycemic control (HbA1C) in the self-management of blood glucose and was published in the American Diabetes Association's own journal, Diabetes Care. The study by Hou et al. found a statistically significant mean reduction in $\mathrm{HbA} 1 \mathrm{c}$ of $0.49 \%$ with a moderate grade of evidence, with younger patients and patients with ongoing healthcare professional support more likely to benefit. Furthermore, the authors concluded that "apps may be an effective component to help control HbA1c and could be considered as an adjuvant intervention to the standard self-management for patients with type 2 diabetes. ${ }^{\prime \prime 0}$

Even in cases where the clinical evidence appears to meet the minimum requirements for inclusion in standard of care guidelines, there are many possible reasons why Digital Health apps are not yet recommended.

Among them are the possibility that guideline writers have reviewed and found the existing evidence base unimpressive; that the evidence base has matured rapidly and has not yet been reviewed; that the traditional communications channels through which guideline writers learn about therapeutics studies have not yet matured in the nascent Digital Health industry, thereby creating a "blind spot" for guideline writers; or the possibility that guideline writers may have unstated requirements - or are unclear on the requirements themselves - for adding a new software-driven treatment modality that presents unfamiliar issues around usability, data protection, feature standardization (e.g., how is a "class" of apps defined), and clinician workflow. While a confluence of these issues are likely in play, patient-centered guideline writers are likely to soon bridge the associated gaps with the Digital Health industry.

For Pear's reSET app, FDA review was based on a multisite controlled trial comparing standard treatment to standard treatment with the addition of reSET. The FDA found that "the data showed a statistically significant increase in adherence to abstinence for the patients with alcohol, cocaine, marijuana and stimulant SUD in those who used reSET, 40.3 percent, compared to the patients who did not, 17.6 percent." ${ }^{187,88}$ In other cases where the FDA has cleared Digital Health apps, the FDA has simply guided the app developers to "be truthful and not misleading" in their marketing, and app developers (like WellDoc) have freely cited their clinical evidence in descriptions of their apps.

\section{Succeeding in a market for outcomes:} merit-based incentives Payment models for Digital Health tools are still evolving. The health system's emerging value-based payment architecture now offers to create a robust market for outcome metrics, but other mechanisms of reimbursement also offer a route to adoption. The Digital Health industry has had some meaningful 
success ensuring clinicians and their patients are appropriately compensated through traditional feefor-service reimbursement models, but not enough to trigger broad adoption across the health system. In these few cases, the Digital Health industry has obtained traditional fee-for-service reimbursement of new Digital Health-enabled services by payers. For example, in 2015 Medicare began paying for Chronic Care Management Services in which clinicians remotely manage patients with chronic conditions and bill for associated services on an ongoing, monthly basis. Similarly, in March 2016 the U.S. Department of Health and Human Services announced coverage of Diabetes Prevention Programs, including not only face-to-face programs but also certain online programs recognized by the Center for Disease Control (e.g., Omada Health, Lark, Vida Health, Wellframe, etc.). However, major program announcements of this kind are few and far between and it's unlikely that the entire diversity of dozens of categories Digital Health solutions will be covered by Medicare.

Product-specific reimbursement - similar to how drugs are reimbursed - has also been attempted with some Digital Health apps lobbying for coverage by specific commercial payers. WellDoc was initially successful in gaining coverage from two insurance companies for its app for self-management of blood glucose in 2011, whereby the app was prescribed by clinicians and access codes were "dispensed" by a specialty pharmacy, enabling covered patients to access the app. Although this model appeared promising, adoption appears to have been modest, with GooglePlay stating that WellDoc BlueStar has only 5,000 - 10,000 installs. ${ }^{91}$

Recent value-based care initiatives, including MACRA, instead have become the greatest incentive for healthcare professionals to use Digital Health products. Legislation tying payments for hospitals, health systems and providers alike to quality metrics such as readmissions and emergency room visits places Digital
"I would say that if you don't have digital medicine and digital therapeutic services in your Digital Health strategy out of an integrated delivery network, you're probably not in business today because of what's required under value based care. You've got to create a stronger relationship with the patient. So, $100 \%$ have either identified it as a strategy or have undertaken a phase one set of pilots or selected tools for deployment. The leaders have begun to fuse those selected apps into their own white label or private label app boutique... [integrated] with their electronic medical system EMR, where they can start to prescribe apps. So, that's a move from passive Digital Health to prescriptive Digital Health where the doctors or members of the care team are now starting to prescribe the app as an extension of the encounter with the healthcare system. They are very few. Those are the leaders."

David Vinson, Founding Director and Vice Chairman, Xcertia and Founder, DHX group 
Health squarely among tools that clinicians can use to manage these and secure higher payments.

Creating efficient clinical workflow: prescribing, data integration and EHR interoperability standards Finally, the focus of health IT tech policy setters on EHR interoperability standards, and other routes to workflow integration, are beginning to bear fruit. As the most incentivized healthcare stakeholder to date, large health systems have led the way in the adoption of Digital Health tools. Even there, adoption is still relatively low. To date, most health systems have invested in Digital Health pilot programs rolled out to a small number of individuals on a trial basis in order to prove the value of the intervention. As Digital Health has become more mature, however, some health systems are now shifting from pilot programs to more full-scale rollouts across sometimes substantial organizations. One vendor estimates that $20 \%$ of healthcare and provider organizations are now moving from pilot projects into program rollouts and of the rest, three quarters are either expanding their pilots or adding a second pilot. One of the largest health systems has additionally begun rolling out a remote patient monitoring program for diabetes and hypertension across several regions and has committed to a nationwide expansion (every region every hospital). ${ }^{92}$

To scale up the use of Digital Health at patient care institutions requires route for clinicians to physically recommend an app to patients, a way to track data (and data quality) associated with the use of Digital Health apps and sensors, and ideally, for information from Digital Health tools to flow into and out of EHR systems. The work of using digital apps and sensors must be made easy.

There is reason to believe that this key challenge will dissolve over time. CMS and other sectors of the healthcare IT industry are increasingly endorsing a new standard FHIR (Fast Healthcare Interoperability Resources) - which enables EHRs to speak more easily to apps. Additionally, vendors such as Validic, Intersystems, and Human API have stepped in to allow healthcare stakeholders to integrate Digital Health tools more rapidly; sometimes enabling across-the-board integration of a menu of digital tools into their EMRs, or simply enabling their data to be used for condition management (see Exhibit 31). These "pipes" take in data

"The question is how do you get patient generated data back into the physician or the clinical work flow system. Most of the EMRs systems ... are closed systems EPIC, Cerner - but there's beginning to be a movement where the larger EMR system, based on market pressure, are beginning to work through integration hubs, companies who are now creating API connectors that's allowing these organizations to move information in without major integration projects. It's slow to happen, but it's happening."

David Vinson, Founding Director and Vice Chairman, Xcertia and Founder, DHX group 
Exhibit 31: Routes to Use of Digital Health Data by Health Systems

\section{ROUTES TO DATA INTEGRATION}

- One-off connectivity built by EMR vendor or Hospital IT staff

- Code written to get data from apps or devices

- Integrates selected Digital health tool only (e.g. Fitbit)

- May use APIs provided by app platforms like HealthKit

- Data may come in as unusable text
- Integration of a menu of tools or specific ones

- Vendor creates a "pipe" to their hub with pre-built APIs and pre-built algorithms translating data value

- Not re-creating the wheel each time

- Available to multiple stakeholders including hospital system, payers, employer

- Messy patient data gets standardized and cleaned before delivery

Source: IQVIA Institute, Sep 2017

Note: API (application program interface); Vendors include Validic, Intersystems, and Human API, among others

from consumer devices, which is then standardized and cleansed, and the EHR provider can then customize how this data is used.

\section{The evolution of infrastructure}

The past few years has seen increasingly sophisticated experimentation by stakeholders who seek to apply new Digital Health tools to transform human health care. Before healthcare can be more fully supported by apps and sensors, however, a new fit-for-purpose infrastructure must evolve to support their delivery and incorporation into the standard health toolkit. Similar to the type of mature drug distribution ecosystem that exists to disseminate safe therapeutics, an infrastructure of quality-comparison tools, institutional procurement processes, prescribing and dispensing tools, payment and incentives management and data management are all needed.

There are now FDA cleared apps that have evidence of benefit similar to that of FDA approved drugs for instance delivering a $1.2 \%$ average reduction in
$\mathrm{HbA1} \mathrm{c}^{93,94}$ comparable to medications for Type 2 Diabetes $^{95}$ - yet none has seen a level of uptake or sales or even stakeholder awareness that is comparable to their pharmaceutical counterparts. No app with evidence of patient benefit has been discussed in guidelines to date. Drugs with similar health impact may be received by healthcare stakeholders more readily in part due to the existence of infrastructure and experience in the pharmaceutical industry that allows standardized prescribing and dispensing, as well as relatively clear guidelines around health plan coverage decisions and reimbursement processes. The existence of these formalized channels to market acceptance also spurs stakeholder investment and financial returns that further enable investment in studies with larger numbers of patients, market access personnel, marketing and sales campaigns that facilitate greater visibility.

Digital Health app developers and technology innovators have successfully produced many good, highly usable, clinically proven apps that create value for patients and the health system. However, it falls 
increasingly to other stakeholders - key policy setters, payers, guideline writers and others - to create the supporting technologies, standards, guidelines and incentives necessary to realize the benefits of Digital Health tools. As the discussion in the Digital Health and Healthcare communities shift towards the requirements for successfully integrating technologies into physician practices, and leaders in all stakeholder groups now begin to collaborate more closely, the use of Digital Health tools within the complex healthcare system will accelerate and create a bright future for innovation in this area.

"I think [in five years] digital health data will be part of the mainstream solution for part of every healthcare organization. We'll be past the point where it's an option or it's in pilot phase. Moving beyond that, we'll see it become a standard part of the engagement with every patient. Whether you are well or have anything specific that they're monitoring, it'll be a part of the way [clinicians] work with every person. That's farther out... in their five to ten year plans. I would be very surprised if by 2020 to 2022... this is not a mainstream component of caring for chronic disease patients and any other patient that needs active monitoring."

Marc Sebes, Vice President of Product Management, Validic 


\section{Appendix}

\section{METHODOLOGY}

\section{Mobile app data}

Global mobile application data was sourced from 42Matters and AppScript in July 2017 and obtained via the AppScript App Database. As of July 11th 2017 when the data was pulled, there were 318,572 health apps available with 166,592 in the Apple Store and 151,980 in the Google Play Store. Although some apps are available on both stores, their unique instances may offer different functionality, and are therefore counted as distinct. Although our 2015 report made use of mobile application data supplied by Mevvy rather than 42Matters, these data sources are believed to be substantially similar, as both suppliers' source data directly from the relevant app stores. Significant differences are not expected, but minor trend breaks may exist and may have minor impact on longitudinal trends.

The analysis only includes apps available for download on the Apple Store and Google Play Store. Other Digital Health apps, including web apps and closed distribution model apps, are not included in the various analyses of app quantity and category but are included in various clinical evidence assessments.

\section{Mobile patient health app data}

Mobile application data sourced from 42Matters was reviewed and supplemented with primary research by IQVIA AppScript to create the curated AppScript App Database of widely available consumer mobile health apps. As of July 11th 2017 when the data was pulled for the 2017 study, a total of 22,357 unique healthcare consumer mobile apps were included in the dataset, including 13,983 iOS apps from the Apple Store and 8,374 Android apps from the Google Play store. This dataset prioritizes review of apps in the "Health and Fitness" and "Medical" categories, as well as the most downloaded apps, to define a set of the Digital Health apps most widely used by consumers. Under
AppScript curation methods, app store apps with greater than 1,000 user ratings are prioritized for in-depth examination, as well as apps that have already been reviewed and have a version or price update. A thorough examination of the content of apps enables exclusion of apps from further analysis that are considered irrelevant to normal healthcare use (e.g., salons, apps with gimmicks, etc.), unavailable in English language, or for healthcare providers as opposed to patients. The remaining included apps are considered genuine Digital Health apps for patients. For the purpose of counting apps, an app may be counted twice if it is available from both the Google Play Store and the Apple App Store; however, differences exist between platforms regarding functionality and download volume.

\section{Android install data analysis}

July 2017 Google Play data contained information on volume of downloads, where downloads were quoted in the following ranges: 10 million to 50 million; 5 million to 10 million; 500,000 to 1 million; 100,000 to 500,000 ; 50,000 to 100,$000 ; 10,000$ to 50,$000 ; 5,000$ to 10,000 ; 1,000 to 5,$000 ; 500$ to 1,$000 ; 100$ to $500 ; 10$ to $50 ; 5$ to $10 ; 1$ to 5 . The median number of downloads was taken for each range, from which a total number of downloads was estimated.

\section{Device data}

Data on available consumer wearable sensors was built using primary research by IOVIA AppScript. As of September 15th 2017 when the data was pulled, a total of 344 unique patient sensors were included in the curated AppScript Device Database.

\section{Clinical trial data}

Data was pulled from clinicaltrials.gov on February 24th 2017. Analysis of clinical trials derived from Clinicaltrials. gov were narrowed to studies with the following recruitment statuses: Active, Recruiting, Enrolling by invitation, Not yet recruiting (only those with a start 
date before Sept 2017 and completion date beyond Sept 2016 OR a start date in 2016 with null completion date and update in $2016 \mathrm{n=10)}$ ), and Unknown status (with a completion date in 2017 or beyond). Trial queries included the following search strings: Mobile application or Mobile app; Smartphone; App in Interventions; App in Title; Android App OR Android Application; iOS App OR iOS Application; Wearable AND mobile; Device AND Mobile. Data was cleansed to exclude trials not involving Digital Health - for instance those looking at amyloid precursor protein (APP), etc. Trials incorporating only passive video materials and DVDs were excluded from the analysis. Trials incorporating tablet based questionnaires and virtual office visits were included in the analysis. For analyses of trial sponsorships: Patient Care includes universities, hospitals and clinics; Industry includes Pharma, Biotech, device and app manufacturers; Foundation includes charities and individuals. The total percent of sponsorships exceed $100 \%$ due to multiple sponsors collaborating in a single trial.

\section{AppScript Score}

The AppScript Score provides a comprehensive method for all stakeholders to assess Digital Health app quality and may be predictive of a given app's value to the human health and the overall health system. The AppScript Score is derived from six sub-scores, or "ratings," across the following dimensions: Patient, Professional, Functional, Developer, Endorsement and Clinical ratings (see Exhibit 16).

Across the six rating, more than 70 individual metrics are considered. Some metrics leverage data from the AppScript distribution platform, which enables clinicians to electronically recommend apps, connected devices and digital content to their patients. AppScript Score components are weighted and combined to generate a consolidated score of 1-100. A "good score" is always at the discretion of a healthcare professional and may vary by not only the condition, but by provider and by patient.
Patient Rating leverages commodity Apple Store and Google Play Store ratings and rating counts as well as well as proprietary AppScript "fill rate" and "retention rate" data pertaining to the number of AppScript app recommendations are downloaded and retained for at least a 30 day period, respectively.

Professional Rating is derived from the number of times a given app is recommended to patients by healthcare professionals using the AppScript platform and the number of times a given app has been included in an institution's Digital Health formulary using the AppScript platform.

Functional Rating measures the feature-set of apps (more detail in Exhibit 4), representing the unique investment by the developer and functionality available to users.

Developer Rating determines the professionalism and dedication of a developer to deliver high-quality apps that leverage the most recent technologies. Key metrics assessed as part of the Developer Rating include the most recent update date of the app and whether the app interoperates with sensors directly or through a data sharing hub (e.g., HealthKit).

Endorsement Rating is based on the number of times a given app has been positively endorsed by credible healthcare organizations such as regulators (e.g., the FDA through a clearance), healthcare provider institutions (e.g., Joslin Clinic), and health content publishers (e.g., HealthLine).

Clinical Rating is an Evidence Based Medicine approach to rating apps focused on the review and scoring of peer reviewed publications. All peer-reviewed publications are scored based on their design qualities and results. Study quality is based on the underlying study design, for example an RCT is scored higher than an observational study. Study result is based on whether the study found that the underlying app provided a statistically significant benefit on a primary endpoint or 
was otherwise found favorable by the study's authors (positive result), showed no statistically significant benefit (neutral result), or was significantly worse than a comparator or was otherwise found unfavorable by the study's authors (negative result). An app's Clinical Rating is the average score of all available peer reviewed publications that have assessed its content, usability, accuracy, efficacy, safety, or underlying health economics.

\section{AppScript Digital Health Evidence Database and Clinical Evidence Maturity Assessment}

Peer-reviewed publications are identified and included in the AppScript Digital Health Evidence Database on a rolling basis leveraging database search as well as manual search methodologies. Google Scholar and PubMed databases are searched on relevant keywords across therapeutics areas, study types and technology categories. The AppScript team also monitors relevant trade publications and industry contacts for new studies which occasionally requires manual entry above and beyond database search methodologies.

The Digital Health App Clinical Maturity Assessment included 571 app efficacy studies included across dozens of app use categories ranging from individual conditions (e.g., diabetes) to prescription management categories (e.g., filling prescriptions). Many types of peer-reviewed publications were not included in the analysis presented as there were no direct and quantitative implications for improved human health, including content review studies, usability studies, technical and clinical accuracy studies, and pure health economic studies (i.e., those based on pre-existing efficacy data). Efficacy studies were categorized by study type (observational, RCT, systematic review or meta-analysis study), outcome (positive, negative, neutral) and primary use category (e.g., therapeutic area/medical condition). Positive study outcomes reflect study results demonstrating statistically significant findings of clinical change.
Studies were summarized via a "Digital Health Clinical Maturity Matrix" (see Exhibit 32). Values for the x-axis, Relative Quantity and Quality of Available Clinical Evidence, were derived via a tiered assessment of the available clinical evidence. The first tier of the assessment was the most heavily weighted and focused on the highest quality study designs currently available for the Digital Health app use category. The study quality point system was scored as follows: No Studies $=0$ points, One Observational Study =1, Multiple Observational Studies $=2$, One RCT $=3$, Multiple RCTs =4, One Meta-Analysis Study = 5, and Multiple MetaAnalysis Studies $=6$. The second tier of the Quantity and Quality assessment was a minor factor focused on study quantity. Total efficacy studies were counted in each Digital Health app use category, with categories with the most studies receiving close to an additional point and all other studies receiving a fraction of a point based on their relative study counts. Values for the y-axis, Average Study Results, are based on averaged individual study result scores, where each individual study is scored a 1.0 (positive), 0.5 (neutral), or 0.0 (negative). For the Average Study Results assessment, only the highest quality available studies were considered. For example, if a given use case category had many observational studies and two RCTs, only the results of the RCTs were considered.

Apps were divided into five clinical maturity groupings based on typical evidence thresholds across the health system. Digital Health app use categories featuring multiple meta-analysis studies and generally positive results were grouped as "Candidates for Inclusion in Clinical Guidelines" as this level of clinical maturity has likely produced studies that meet the explicitly stated requirements of clinical guideline writers. Categories with at least one RCT and generally positive results were grouped as "Candidates for Adoption" as health plans, healthcare providers and individual clinicians generally regard RCT data as a gold standard evaluation. Categories with only observational studies were grouped 


\section{Exhibit 32: Digital Health Clinical Maturity Matrix}

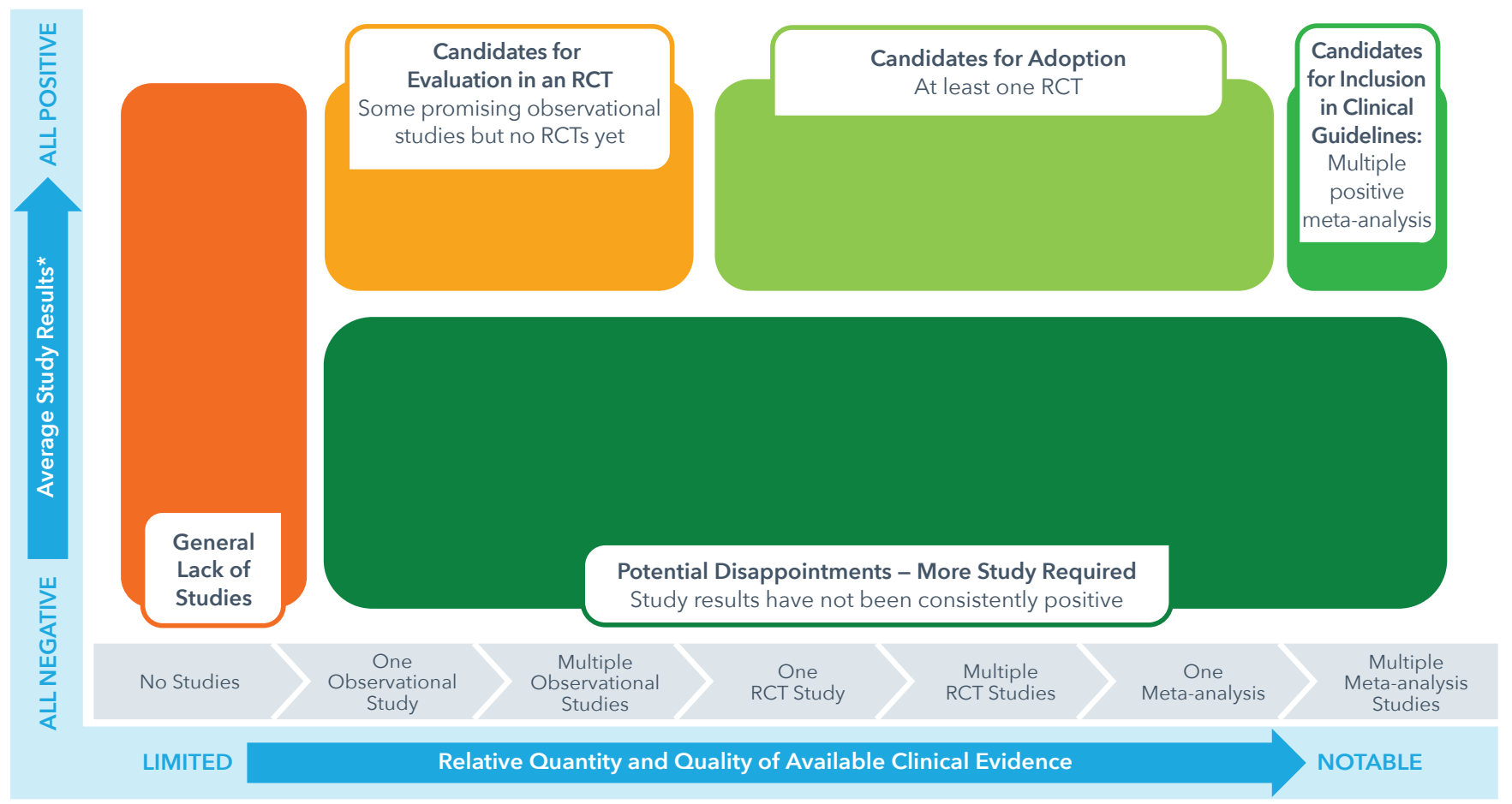

Sources: AppScript Clinical Evidence Database, Aug 2017

Notes: Only includes studies that evaluated the interventional value of a digital health solution (mobile or web app, connected device or other mobile intervention such as texting) on patient outcomes such as activity levels, lab results, or healthcare resource utilization

*Average of study results for the highest quality evidence available (i.e., meta-analysis > RCT > Observational)

as "Candidates for Evaluation in an RCT" as they may be considered sufficiently de-risked to invest in a robust, gold-standard RCT study. Categories without any efficacy studies were grouped as "General Lack of Studies." Categories with average results of $\sim 0.6$ or lower (i.e., at best, closer to neutral than positive) were grouped as "Potential Disappointments - More Study Required" as key health system stakeholders generally expect new healthcare interventions to consistently demonstrate significant clinical value when studied. This being said, given the broad capacity of Digital Health to improve human health outcomes demonstrated in this report, it is likely that many of these "Potentially Disappointing" categories will ultimately find the appropriate functionality, delivery models and patient sub-populations where consistently favorable results are possible.

\section{AppScript Essentials Value Model}

The AppScript Essentials Value Model is an evolving health economic model that estimates the potential value of available Digital Health apps in terms of clinical outcomes and cost reduction based on a bottom up analysis clinical benefits identified in available peerreviewed publications and extrapolation of identified clinical benefits to appropriate patient populations.

In the version of the AppScript Essentials Value Model published in this report (v1.0), a search for favorable acute care utilization findings across priority use cases for Digital Health apps was executed, resulting in the 
identification of 5 key studies. First, each study's patient populations and clinical benefits were considered (see Exhibit 33).

Second, an appropriate model was developed starting with the target patient population defined by underlying epidemiology and considering factors such as smartphone ownership (ability to use) likely engagement rates (likelihood to download and/or benefit) based on available evidence and experience from the AppScript team (see Exhibit 34).

\section{Exhibit 33: Studies Used to Develop the AppScript Essentials Value Model}

\begin{tabular}{|c|c|c|c|c|c|}
\hline $\begin{array}{l}\text { USE CASE } \\
\text { CATEGORY }\end{array}$ & APP & STUDY & POPULATION & METHODOLOGY & KEY FINDING \\
\hline $\begin{array}{l}\text { Diabetes } \\
\text { Prevention }\end{array}$ & $\begin{array}{l}\text { Omada } \\
\text { Health }\end{array}$ & $\begin{array}{l}\text { Chen et } \\
\text { al (2016) }\end{array}$ & $\begin{array}{l}>65 y \text { Prediabetics: 1,121 overweight } \\
\text { or obese seniors with additional risk } \\
\text { factors for diabetes or heart disease }\end{array}$ & $\begin{array}{l}\text { Health } \\
\text { Economic } \\
\text { Model based } \\
\text { on previous } \\
\text { experience }\end{array}$ & $\begin{array}{l}\text { Reduction in long-term diabetes incidence and } \\
\text { associated healthcare utilization costs across } \\
\text { hospitalizations, ED visits, ambulatory care, } \\
\text { and pharmacy net of intervention costs ranging } \\
\text { from } \$ 1,201 \text { - } \$ 1,454 \text { per capita per year }\end{array}$ \\
\hline Diabetes & WellDoc & $\begin{array}{l}\text { Katz et al } \\
(2012)\end{array}$ & $\begin{array}{l}\text { Diabetics: } 32 \text { patients (100\% African } \\
\text { American; } 97 \% \text { women) enrolled with } \\
\text { prestudy mean HgA1c of } 8 \%\end{array}$ & $\begin{array}{l}\text { Observational } \\
\text { Study: Pre- } \\
\text { post design }\end{array}$ & $\begin{array}{l}\text { Reduction in ED and hospitalization rate: } \\
\text { - } 16(50 \%) \text { became active users } \\
\text { - } ~ 58 \% \text { reduction in ED + Hospital } \\
\text { Admissions for Active Group (including } 0 \\
\text { hospitalizations) } \\
\text { - All diabetes care measures improved }\end{array}$ \\
\hline Asthma & $\begin{array}{l}\text { Propeller } \\
\text { Health }\end{array}$ & $\begin{array}{l}\text { Merchant } \\
\text { et al } \\
(2017)\end{array}$ & $\begin{array}{l}\text { Asthmatics: } 330 \text { Propeller Health } \\
\text { users with an average of } 647 \text { baseline } \\
\text { (pre-intervention) and } 312 \text { post- } \\
\text { intervention days }\end{array}$ & $\begin{array}{l}\text { Observational } \\
\text { Study: Pre- } \\
\text { post design }\end{array}$ & $\begin{array}{l}\text { Reduction in all cause hospitalization, ED visits, } \\
\text { and physician office utilization as follows: } \\
\text { - } \sim 34 \% \text { lower hospital admission rate } \\
\text { - } \sim 23 \% \text { lower ED visit rate } \\
\text { - } \sim 4 \% \text { lower physician office visit rate }\end{array}$ \\
\hline $\begin{array}{l}\text { Cardiac } \\
\text { Rehab (CR) }\end{array}$ & $\begin{array}{l}\text { Healarium } \\
\text { (Mayo } \\
\text { Clinic } \\
\text { instance) }\end{array}$ & $\begin{array}{l}\text { Widmer } \\
\text { et al } \\
(2017)\end{array}$ & $\begin{array}{l}\text { MI: Patients after percutaneous } \\
\text { coronary intervention }(\mathrm{PCI}) \text { for acute } \\
\text { coronary syndrome (ACS) }\end{array}$ & $\begin{array}{l}\text { RCT: Digital } \\
\text { Health } \\
\text { Intervention } \\
\text { (DHI) vs. face- } \\
\text { to-face CR }\end{array}$ & $\begin{array}{l}\text { Strong trends towards fewer patients visiting } \\
\text { ED or being admitted to hospital within } 180 \\
\text { days [CV-related rehospitalizations plus ED } \\
\text { visits compared to the control group at } 180 \\
\text { days ( } 8.1 \% \text { vs } 26.6 \% \text {; RR } 0.30,95 \% \mathrm{Cl} 0.08-1.10 \text {, } \\
\mathrm{P}=.054) .]\end{array}$ \\
\hline $\begin{array}{l}\text { Pulmonary } \\
\text { Rehab (PR) }\end{array}$ & MyCOPD & $\begin{array}{l}\text { Bourne } \\
\text { et al } \\
(2017)\end{array}$ & $\begin{array}{l}\text { COPD: } 90 \text { patients with a diagnosis } \\
\text { of chronic obstructive pulmonary } \\
\text { disease (COPD), modified Medical } \\
\text { Research Council score of } 2 \text { or } \\
\text { greater referred for pulmonary } \\
\text { rehabilitation }\end{array}$ & $\begin{array}{l}\text { RCT: Digital } \\
\text { Health } \\
\text { Intervention } \\
\text { (DHI) vs. face- } \\
\text { to-face PR }\end{array}$ & $\begin{array}{l}\text { The study demonstrated that the } \mathrm{DHI} \text { is } \\
\text { non-inferior to face-to-face PR, which has } \\
\text { demonstrated value in preventing acute care } \\
\text { utilization }\end{array}$ \\
\hline
\end{tabular}




\section{Exhibit 34: Methodology for Estimating Cost Reduction in the AppScript Essentials Value Model}

\begin{tabular}{|c|c|}
\hline $\begin{array}{l}\text { USE CASE } \\
\text { CATEGORY }\end{array}$ & METHODOLOGY FOR ESTIMATING COST REDUCTION \\
\hline $\begin{array}{l}\text { Diabetes } \\
\text { Prevention }\end{array}$ & $\begin{array}{l}\text { 1. Considered patient population and cost savings included in Chen et al (2016) and concluded that the study only considers } \\
\text { benefits across active users as opposed to an entire population of prediabetics } \\
\text { 2. Collected estimates for }>65 \text { prediabetic population } \\
\text { 3. Multiplied by estimated }>65 \text { yo population that own smart phone (rationale: smart phone users are the likely target audience) } \\
\text { 4. Multiplied by estimated \% that may "fill" a digital health prescription upon distribution in an outpatient environment } \\
\text { 5. Multiplied by annualized per capita cost benefits (used an average of two models introduced in (Chen et al) } \\
\text { 6. Calculated total cost savings potential }\end{array}$ \\
\hline Diabetes & $\begin{array}{l}\text { 1. Considered patient population and acute care utilization benefits in Katz et al (2012) and concluded that the intervention was } \\
\text { applied to a patient population that was sicker than the overall diagnosed diabetic population, but less sick than recently } \\
\text { hospitalized diabetics, suggesting that the benefits could likely be estimated by looking at cost savings in (A) the overall } \\
\text { diabetic population and (B) recently hospitalized diabetics and averaging } \\
\text { 2. Collected estimates for diagnosed diabetic population and unique hospitalized diabetics } \\
\text { 3. Multiplied by engagement rate from Katz et al study } \\
\text { 4. Applied acute care utilization benefits from Katz et al study to each of the two populations (note: in calculating status quo ED } \\
\text { visit and hospitalization rates, only diabetes-specific utilization was considered as opposed to all utilization with a diabetes } \\
\text { diagnosis. This method resulted in a far more conservative estimate) } \\
\text { 5. Multiplied by cost of ED visits, hospital admissions, etc. } \\
\text { 6. Calculated total cost savings potential }\end{array}$ \\
\hline Asthma & $\begin{array}{l}\text { 1. Considered patient population and acute care utilization benefits in Merchant et al (2017) and concluded patient population } \\
\text { was fairly representative of overall asthma population, however, the benefits were in engaged users (i.e., the study does not } \\
\text { consider the fact that not all patients introduced to the intervention would initiate it) } \\
\text { 2. Collected estimates for diagnosed asthma population (all ages) } \\
\text { 3. Multiplied by engagement rate ("fill rate") from AppScript experience in outpatient digital health prescribing } \\
\text { 4. Applied acute care utilization benefits from Merchant et al study } \\
\text { 5. Multiplied by cost of ED visits, hospital admissions, etc. } \\
\text { 6. Calculated total cost savings potential }\end{array}$ \\
\hline
\end{tabular}

1. Considered patient population and acute care utilization benefits in Widmer et al (2017) and concluded it may be reasonable to target the intervention (and associated benefits) to the total number of unique patients hospitalized for myocardial infarction (MI) in a given year

2. Collected estimates for unique patients hospitalized for $\mathrm{MI}$ each year

3. Applied acute care utilization benefits from Widmer et al study

4. Multiplied by cost of ED visits, hospital admissions, etc.

5. Calculated total cost savings potential

1. Considered patient population in Bourne et al (2017) as well as manufacturers list price for intervention relative to estimates of face-to-face pulmonary rehab costs and concluded that the MyCOPD intervention is being applied to patients currently being referred to pulmonary rehab and cost savings should primarily be a function of the app's clinical non-inferiority and lower cost

2. Estimated total pulmonary rehab referrals taking place each year

3. Multiplied by estimated $>65 y$ o population that own smart phone (rationale: smart phone users are the likely target audience)

4. Multiplied by cost savings associated with app-based PR vs. face-to-face

5. Calculated total cost savings potential 


\section{References}

1. WHO. mHealth: New horizons for health through mobile technologies. Global Observatory for eHealth Series - Volume 3. 2011

2. HIMSS. Definitions of mHealth. 2012 Jan 5. Available from: http://www.himss.org/definitions-mhealth

3. Pew Research Center. Internet \& technology mobile fact sheet. 2017 Jan 12. Available from: http://www. pewinternet.org/fact-sheet/mobile/

4. Apple Inc. Website. App Store Tab. Available from: https://developer.apple.com/app-store/responding-toreviews/

5. Android.com Website. Developers Blog. Available: https://android-developers.googleblog.com/2013/05/ all-google-play-developers-can-now.html

6. Perez S. Apple's big App Store purge is now underway. 2016 Nov. 15. Available from: https://techcrunch. com/2016/11/15/apples-big-app-store-purge-is-now-underway/

7. Gartenberg C. The Verge. Apple officially bans scammy antivirus apps from iOS App Store. 2017 Sep 15. Available from: https://www.theverge.com/2017/9/15/16314034/apple-developer-guidelines-updatescam-anti-virus-apps-banned

8. Aadeetya S. Google to pull the plug on low quality apps from Play store. The Quint. 2017 Aug 5. Available from: https://www.thequint.com/tech-and-auto/tech-news/google-to-remove-apps-from-play-store-toimprove-android

9. Samsung. Samsung bio-processor for health monitoring. 2016. Available from: http://www.samsung.com/ us/samsungsemiconductor/pdfs/health-monitoring-whitepaper.pdf

10. Sawh M. Samsung S-Patch 3 could deliver serious health monitoring. Wareable. 2017 Jun 7. Available from: https://www.wareable.com/samsung/samsung-s-patch-3-release-date-price-specs-4609

11. Freedman B. Screening for atrial fibrillation using a smartphone: is there an app for that?. Journal of the American Heart Association. 2016 Jul 21. 2016;5(7) Available from: https://doi.org/10.1161/ JAHA.116.004000

12. Chan PH, Wong CK, Poh YKC, Pun L, Leung WW, Wong YF, et al. Diagnostic performance of a smartphonebased photoplethysmographic application for atrial fibrillation screening in a primary care setting. Journal of the American Heart Association. 2016 Jul 21. 2016;5(7) Available from: https://doi.org/10.1161/ JAHA.116.003428

13. Vaid J, Poh MZ, Saleh A, Kalantarian S, Poh YKC, Rafael A, et al. Diagnostic accuracy of a novel mobile application (Cardiio Rhythm) for detecting atrial fibrillation. 2015 Mar 17. J American College of Cardiology;65(10 Supp):A361. Available from: https://doi.org/10.1016/S0735-1097(15)60361-8 
14. FDA. Mobile medical applications. Cited on 2017 Sep. Available from: https://www.fda.gov/ MedicalDevices/DigitalHealth/MobileMedicalApplications/default.htm\#b

15. Mohammadi D. The Pharmaceutical Journal. Smart inhalers: will they help to improve asthma care? 2017 Apr 7. http://www.pharmaceutical-journal.com/news-and-analysis/features/smart-inhalers-will-they-helpto-improve-asthma-care/20202556.article

16. Comstock J. Teva Pharmaceuticals buys smart inhaler company Gecko Health Innovations. 2015 Sep 27. Available from: http://www.mobihealthnews.com/47039/teva-pharmaceuticals-buys-smart-inhalercompany-gecko-health-innovations

17. Medtech Insight. New data gives Adherium fuel to accelerate digital tech adoption in drug sector to improve outcomes for children with poorly controlled asthma. 2016 Nov 19. Available from: http:// adherium.com/media/1130/19nov16_medtech-insight.pdf

18. D'arcy S, MacHale E, Seheult J et al. A method to assess adherence in inhaler use through analysis of acoustic recordings of inhaler events. PLoS ONE 2014;9:e98701. Available from: doi: 10.1371/journal. pone.0098701

19. INhaler Compliance Assessment. Current Studies. Accessed 1 Sept 2017. Available from: http://www. incadevice.com/clinical-trials/

20. Startup Nation Finder. [Business Listing] Breathe.me Asthma and COPD monitoring and management. Accessed 2017 Sep 19. Available from: https://finder.startupnationcentral.org/c/breathe-me

21. García-Pérez L-E, Álvarez M, Dilla T, Gil-Guillén V, Orozco-Beltrán D. Adherence to therapies in patients with type 2 diabetes. Diabetes Therapy. 2013;4(2):175-194

22. Comstock J. In-Depth: Five innovators who see the future of connected insulin delivery in pens, not pumps. Mobihealthnews. Internet. 2016 Oct 21. Available from: http://www.mobihealthnews.com/content/ depth-five-innovators-who-see-future-connected-insulin-delivery-pens-not-pumps

23. Rens N. Validating Digital Health: Interview with Deborah Kilpatrick, Ph.D. Hopkins Biotech Network. 2015 Jun 3. Available from: http://hopkinsbio.org/biotechnology/validating-digital-health-deborah-kilpatrick/

24. Call for Papers. The 1st workshop on digital biomarkers 2017. ACM Mobisys 2017 Workshop. Niagara Falls NY. 2017 Jun 23. http://pac.cs.cornell.edu/wdb2017/papers/CFP.pdf

25. Alam R, Gong J, Hanson M, Bankole A, Anderson M, Smith-Jackson T, et al. Motion biomarkers for early detection of dementia-related agitation. In Proceedings of the 1st Workshop on Digital Biomarkers (DigitalBiomarkers '17). ACM, New York, NY, USA, 15-20. Available from: https://doi. org/10.1145/3089341.3089344 


\section{References}

26. Hurley D. Smartphone monitoring of PD symptoms proves accurate in clinical trial. News from the International Congress of Parkinson's Disease and Movement Disorders. Neurology Today. 2017 Jul 6

27. Fox Insight. Fox Insight Wearables: a virtual research study. A study of The Michael J. Fox Foundation. Accesssed 2017 Sep 19. Available from: https://www.michaeljfox.org/fox-insight-form.html

28. Lipsmeier F, Fernandez Garcia I, Wolf D, Kilchenmann T, Scotland A, Schjodt-Eriksen J, et al. Successful passive monitoring of early-stage Parkinson's disease patient mobility in Phase I RG7935/PRX002 clinical trial with smartphone sensors [abstract]. Mov Disord. 2017; 32 (suppl 2).

29. ClinicalTrials.gov. Mobile Parkinson observatory for worldwide, evidence-based research (mPower). Last updated $2016 \mathrm{Jul} 25$. Available from: https://clinicaltrials.gov/show/NCT02696603

30. ClinicalTrials.gov. Mobile phone sensing and outreach as adjuncts to internet-based behavioral intervention for depression. Last updated 2017 Mar 24. Available from: https://clinicaltrials.gov/show/ NCT01107041

31. Huang Y, Gong J, Rucker M, Chow P, Fua K, Gerber MS, et al. 2017. Discovery of behavioral markers of social anxiety from smartphone sensor data. In Proceedings of the 1st Workshop on Digital Biomarkers (DigitalBiomarkers '17). ACM, New York, NY, USA, 9-14. DOI: Available from: https://doi. org/10.1145/3089341.3089343.

32. Moon Y, McGinnis RS, Seagers K, Motl RW, Sheth N, Wright JA, Jr., et al. Monitoring gait in multiple sclerosis with novel wearable motion sensors. 2017. PLoS ONE 12(2): e0171346.

33. Sehgal S, Chowdhury A, Rabih F, Gadre A, Highland KB. Fitbit vs six minute walk in PAH. [Meeting Abstract] B28. In: Let's go crazy: exercise responses and physiological assessment in pulmonary vascular disease A3110-A3110. Available from: http://www.atsjournals.org/doi/abs/10.1164/ajrccm-conference.2017.195.1_ MeetingAbstracts.A3110

34. ClinicalTrials.gov. Smartphone delivered in-home cardiopulmonary rehabilitation. Last updated: December 28, 2016. Accessed 2017 Sep 5. Available from: https://clinicaltrials.gov/show/NCT02791685

35. Comstock J. Sunovion taps Empatica for Phase 4 drug trial -- and two other Digital Health projects targeting epilepsy. Mobihealth News. 2016 Dec 5. Available from: http://www.mobihealthnews.com/ content/sunovion-taps-empatica-phase-4-drug-trial-and-two-other-digital-health-projects-targeting

36. Interview with Christine Lemke, President, Evidation Health. 2017 Mar 7.

37. Ferguson, $T$ et al. The validity of consumer-level, activity monitors in healthy adults worn in free-living conditions: a cross sectional study. Int J Behav Nut Phys Act. 2015; $12: 42$ 
38. Lee HA, Lee HJ, Moon JH, et al. Comparison of wearable activity tracker with Actigraphy for sleep evaluation and circadian rest-activity rhythm measurement in healthy young adults. Psychiatry Investigation. 2017;14(2):179-185.

39. U.S. Food and Drug Administration. News \& Events. Speeches by FDA Officials. Dr. Gottlieb's speech to the 2017 AdvaMed (MedTech) Conference. 2017 Sep 26. Available from: https://www.fda.gov/NewsEvents/ Speeches/ucm577416.htm

40. Comstock J. Apple, Fitbit, Samsung, and Verily among FDA's picks for precertification pilot. 2017 Sep 26. Available from: http://www.mobihealthnews.com/content/apple-fitbit-samsung-and-verily-among-fdaspicks-precertification-pilot

41. Battelle Technology Partnership Practice Prepared for Pharmaceutical Research and Manufacturers of America (PhRMA). Biopharmaceutical industry-sponsored clinical trials: impact on state economies. 2015 Mar

42. Eastern Research Group, Inc. submitted to U.S. Department of Health and Human Services. Examination of clinical trial costs and barriers for drug development. $2014 \mathrm{Jul} 25$. Available from:https://aspe.hhs.gov/ report/examination-clinical-trial-costs-and-barriers-drug-development

43. Quisel T, Foschini L, Signorini A, Kale DC. KDD 2017. 2017 Aug 13-17. Applied Data Science Paper. Collecting and analyzing millions of mhealth data streams. Available from: http://www.evidation.com/ assets/collecting-and-analyzing-millions-of-mhealth-data-streams.pdf

44. ClinicalTrials.gov. Use of mobile devices and the internet to streamline an asthma clinical trial (MICT). Last updated 2017 Mar 28. Available from: https://clinicaltrials.gov/show/NCT02061280

45. Al Cure Website. Clinical Research. Available from: https://aicure.com/clinical-research/

46. Discussion with Gregory T. Plante, Principal, Digital Health \& Technology, IQVIA. 2017 Aug 3.

47. AstraZeneca. AstraZeneca and Voluntis to test companion mobile app in ovarian cancer studies with the US National Cancer Institute. 2015 Dec 7. Available from: https://www.astrazeneca.com/media-centre/ press-releases/2015/AstraZeneca-and-Voluntis-to-test-companion-mobile-app-in-ovarian-cancerstudies-07122015.html

48. Bain EE, Shafner L, Walling DP, Othman AA, Chuang-Stein C, Hinkle J, Hanina A. Use of a novel artificial intelligence platform on mobile devices to assess dosing compliance in a Phase 2 clinical trial in subjects with schizophrenia. JMIR Mhealth Uhealth 2017;5(2):e18. Available from: https://mhealth.jmir.org/2017/2/e18/

49. Blaschke TF1, Osterberg L, Vrijens B, Urquhart J. Adherence to medications: insights arising from studies on the unreliable link between prescribed and actual drug dosing histories. Annu Rev Pharmacol Toxicol. 2012; 52:275-301 


\section{References}

50. QuintilesIMS. AccessPoint Wearables Podcast \#2. [podcast]. 2017 Aug 14. Available from: https://www. buzzsprout.com/7541/551523-accesspoint-wearables-podcast-2?sf61420077=1

51. Eissa MA, Poffenbarger T, Portman RJ. Comparison of the Actigraph versus patients' diary information in defining circadian time periods for analyzing ambulatory blood pressure monitoring data. Blood Press Monit. 2001 Feb; 6(1): 21-25. https://www.ncbi.nlm.nih.gov/pubmed/11248757

52. Evidation Health. Evidation Health selects PokitDok to help accelerate clinical studies in digital health. 2015 May 12. Available from: http://www.evidation.com/news/press-releases/evidation-health-selectspokitdok-to-help-accelerate-clinical-studies-in-digital-health/

53. Interview with Nelia Padilla, VP, Advisory Services, IQVIA. 2017 Feb 1.

54. Comstock J. Clinical Trials Transformation Initiative releases guidelines on mobile-derived novel endpoints. Mobihealth News. 2017 Jun 26. Available from: http://www.mobihealthnews.com/content/ clinical-trials-transformation-initiative-releases-guidelines-mobile-derived-novel-endpoints

55. Porter ME, Teisberg EO. Redefining health care: creating value-based competition on results. Boston: Harvard Business School Press, 2006

56. Berwick DM, Nolan TW, Whittington J. The Triple Aim: care, health, and cost. Health Aff (Milwood). 2008; 27(3):759-69

57. Accenture. Accenture consumer survey on patient engagement. 2013 Sep. Available from: https://www. accenture.com/t20150708T033734__w__/us-en/_acnmedia/Accenture/Conversion-Assets/DotCom/ Documents/Global/PDF/Industries_11/Accenture-Consumer-Patient-Engagement-Survey-US-Report.pdf

58. Interview with Greg Orr, VP, Digital Health, Walgreens Boots Alliance. August 16, 2016

59. American Medical Association. Digital Health study physicians' motivations and requirements for adopting digital clinical tools. [Survey]. 2016 Sep 26. Available from: https://www.ama-assn.org/sites/default/files/ media-browser/specialty\%20group/washington/ama-digital-health-report923.pdf

60. Volpp K, Mohta N. Patient engagement survey: technology tools gain support - but cost is a hurdle. NEJM Catalyst Insights Report. 2017 Jan 26. Available from: http://catalyst.nejm.org/patient-engagementtechnology-tools-gain-support/

61. Under Armour. Website. Accessed 2017 Sep 18. Available from: http://advertising.underarmour.com/\#top

62. IMS Institute for Healthcare Informatics. Patient apps for improved healthcare: from novelty to mainstream. 2013 Oct

63. IMS Institute for Healthcare Informatics. Avoidable costs in U.S. healthcare: The $\$ 200$ billion opportunity from using medicines more responsibly. IMS Institute for Healthcare Informatics. 2013 Jun. 
64. IMS Institute for Healthcare Informatics. Advancing the responsible use of medicines: applying levers for change. 2012 Oct

65. luga AO, McGuire MJ. Adherence and health care costs. Risk Management and Healthcare Policy. 2014;7:35-44

66. Monitoring Pre-exposure Prophylaxis for Young Adult Women (MPYA). Clinical trial details. Accessed 2017 Sep 1. Available from: https://clinicaltrials.gov/show/NCT02915367

67. Proteus Digital Health. Oracle and Proteus integrate Proteus digital feedback system with Oracle Health Sciences InForm to help increase clinical trial accuracy. 2015 Jan 12. Available from: http://www.proteus. $\mathrm{com} /$ press-releases/oracle-and-proteus-integrate-proteus-digital-health-feedback-system-with-oraclehealth-sciences-inform-to-help-increase-clinical-trial-accuracy/

68. Wade RL, Clancy B, Michaeli J. Improvement in antihypertensive and cholesterol-lowering medication persistence using a mobile technology application. Value in Health 2016; 19(3): A306 (abstract PMD53).

69. IMS Institute for Healthcare Informatics. Patient adoption of mHealth. $2015 \mathrm{Sep}$

70. Standards of medical care in diabetes - 2017. American Diabetes Association. 2017

71. ClinicalTrials.gov. Monitoring of multiple sclerosis (MS) participants with the use of digital technology (Smartphones and Smartwatches) - A Feasibility Study. 2017 May. Available from: https://clinicaltrials.gov/ show/NCT02952911

72. ClinicalTrials.gov. Accu-Chek Connect at school (CATS) pediatric study (CATS). Last Update Posted: 2017 Jul. Available from: https://clinicaltrials.gov/show/NCT02609633

73. ClinicalTrials.gov. ACCU-CHEK Connect personal diabetes management study (PDM CONNECT). 2017 Jun. Available from: https://clinicaltrials.gov/show/NCT02600845

74. ClinicalTrials.gov. A study to evaluate the effects of Basmisanil in participants with cognitive impairment associated with schizophrenia (CIAS) treated with antipsychotics. 2017 Sep. Available from: https:// clinicaltrials.gov/show/NCT02953639

75. ClinicalTrials.gov. A Closed-loop assessment and treatment platform for mood disorders. 2017 Jun. Available from: https://clinicaltrials.gov/show/NCT02948036

76. ClinicalTrials.gov. Piloting a novel, mobile cognitive training tool for patients with major depressive disorder (MDD). 2017 Sep. Available from: https://clinicaltrials.gov/show/NCT02922556

77. ClinicalTrials.gov. Piloting a novel, mobile cognitive tracking and training tool for patients with Parkinson's disease (PD). 2017 Feb. Available from: https://clinicaltrials.gov/show/NCT02922530 


\section{References}

78. ClinicalTrials.gov. Home-based, online, mindfulness and cognitive training for soldiers and veterans with TBI (CogMind). 2017 May. Available from: https://clinicaltrials.gov/show/NCT02922569

79. ClinicalTrials.gov. Digital health feedback system for longitudinal measurement of medication adherence during initial ARV therapy. 2016 Jun. Available from: https://clinicaltrials.gov/show/NCT02800655

80. ClinicalTrials.gov. Wirelessly observed therapy in comparison to directly observed therapy for the treatment of tuberculosis. 2017 Aug. Available from: https://clinicaltrials.gov/show/NCT01960257

81. Walters T, Parcher B, Kravetz A, Campbell C, Shah M, Hale T. Appraising the value of digital health technologies from the managed care perspective: insights for evidence assessment and reimbursement in the United States. Xcenda. Journal of Medical Internet Research. Dec 2016;18(12):1

82. American Medical Association. AMA CEO outlines digital challenges, opportunities facing medicine. 2016 Jun 11. Available from: https://www.ama-assn.org/ama-ceo-outlines-digital-challenges-opportunitiesfacing-medicine

83. Future of Privacy Forum. FPF mobile apps study. 2016 Aug. Available from: https://fpf.org/wp-content/ uploads/2016/08/2016-FPF-Mobile-Apps-Study_final.pdf

84. Blennar et al. Privacy policies of android apps and sharing of health information. JAMA. 2016;315(10): 1051-1052

85. 5th Annual state of mobile application security report: perception vs. reality. Arxan. 2016 Jan.

86. Interview with David Vinson, Founding Director and Vice Chairman of Xcertia and Founder of DHX group. 2017 Aug 8

87. Krueger AC. RE: DEN160018. Received by: Nandini Murthy, Pear Therapeutics, Inc. 2017 Sep 14. Available from: https://www.accessdata.fda.gov/cdrh_docs/pdf16/DEN160018.pdf

88. FDA News Release: FDA permits marketing of mobile medical application for substance use disorder. 2017 Sep 14. Available from: https://www.fda.gov/NewsEvents/Newsroom/PressAnnouncements/ucm576087.htm

89. American Diabetes Association. Position Statement: Standards of Medical Care in Diabetes - 2017. Diabetes Care 2017;40(Suppl. 1):S1-S138 
90. Hou C, Carter B, Hewitt J, Francisa T, Mayor S. Do mobile phone applications improve glycemic control $(\mathrm{HbA} 1 \mathrm{c})$ in the self-management of diabetes. a systematic review, meta-analysis, and grade of 14 randomized trials. 2016 Nov. Available from: https://doi.org/10.2337/dc16-0346

91. Google Play. Accessed 2017 Sep 25. Available from: https://play.google.com/store/apps/details?id=com. welldoc.platform.android\&hl=en

92. From an interview with Marc Sebes, VP of Product Management, Validic. 2017 Mar 3

93. U.S. Food and Drug Administration. The Center for Devices and Radiological Health (CDRH). Welldoc: Response to FDA Feedback on 510(K) KI00066. 2010 Jun 10. Available from: https://www.accessdata.fda. gov/cdrh_docs/pdf10/K100066.pdf

94. Quinn CC, Shardell MD, Terrin MT, Barr EA, Ballew SH, Gruber-Baldini AL. Cluster-randomized trial of a mobile phone personalized behavioral intervention for blood glucose control. Diabetes Care. 2011 Sep; 34(9): 1934-1942

95. Invokana. Healthcare provider, clinical results, monotherapy, A1c. Accessed 2017 Oct 14. Available from: https://www.invokanahcp.com/clinical-trial-results/monotherapy/a1c-change 


\section{About the authors}

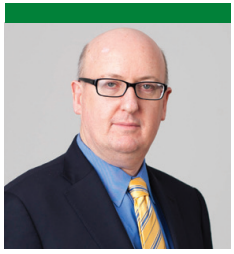

MURRAY AITKEN

Executive Director, IQVIA Institute

for Human Data Science

Murray Aitken is Executive Director, IQVIA Institute for Human Data

Science, which provides policy setters and decisionmakers in the global health sector with objective insights into healthcare dynamics. He led the IMS Institute for Healthcare Informatics, now the IQVIA Institute, since its inception in January 2011. Murray previously was Senior Vice President, Healthcare Insight, leading IMS Health's thought leadership initiatives worldwide. Before that, he served as Senior Vice President, Corporate Strategy, from 2004 to 2007. Murray joined IMS Health in 2001 with responsibility for developing the company's consulting and services businesses. Prior to IMS Health, Murray had a 14-year career with McKinsey \& Company, where he was a leader in the Pharmaceutical and Medical Products practice from 1997 to 2001. Murray writes and speaks regularly on the challenges facing the healthcare industry. He is editor of Health $\mathrm{IQ}$, a publication focused on the value of information in advancing evidencebased healthcare, and also serves on the editorial advisory board of Pharmaceutical Executive. Murray holds a Master of Commerce degree from the University of Auckland in New Zealand, and received an M.B.A. degree with distinction from Harvard University.

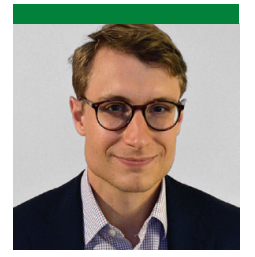

\section{BRIAN CLANCY}

Senior Product Manager, AppScript Analytics, IOVIA

Brian Clancy is Senior Product Manager, IOVIA AppScript Analytics, which provides technology-enabled information and services for Digital Health app curation, market insights, and evidence development. Prior to AppScript, Brian had a seven-year career as a strategy consultant to leading and emerging health technology and pharmaceutical companies. He is an author of various academic and industry publications on personalized medicine and digital health, he is an inventor in the field of app curation and a frequent conference speaker on the topic of technical innovation in healthcare. Brian holds Bachelor of Science and Bachelor of Arts degrees in Molecular Genetics and Economics from the University of Rochester.

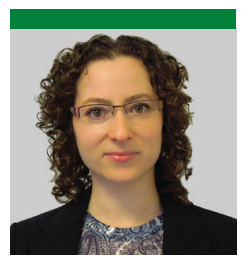

\section{DEANNA NASS}

Director of Publications, IQVIA Institute for Human Data Science

Deanna Nass is the director of publications at the IQVIA Institute for Human Data Science. She manages the development and production lifecycles of IOVIA Institute reports and performs analyses of global biopharmaceutical and healthcare trends. With a diverse background that spans from consulting and business development to market analysis and writing industry publications, she brings a unique perspective of the biopharma industry to the Institute. Deanna joined the Institute in 2013 and IMS Health in 2004. Deanna holds a B.A. in Biology from Yale University with a specialization in Neurobiology and a Certificate in International Affairs from New York University. 


\section{About the IOVIA Institute}

The IQVIA Institute for Human Data Science contributes to the advancement of human health globally through timely research, insightful analysis and scientific expertise applied to granular non-identified patient-level data.

Fulfilling an essential need within healthcare, the Institute delivers objective, relevant insights and research that accelerate understanding and innovation critical to sound decision making and improved human outcomes. With access to IQVIA's institutional knowledge, advanced analytics, technology and unparalleled data the Institute works in tandem with a broad set of healthcare stakeholders to drive a research agenda focused on Human Data Science including, including government agencies, academic institutions, the life sciences industry and payers.

\section{RESEARCH AGENDA}

The research agenda for the Institute centers on five areas considered vital to contributing to the advancement of human health globally.

- Improving decision-making across health systems through the effective use of advanced analytics and methodologies applied to timely, relevant data.

- Addressing opportunities to improve clinical development productivity focused on innovative treatments that advance healthcare globally.

- Optimizing the performance of health systems by focusing on patient centricity, precision medicine and better understanding disease causes, treatment consequences and measures to improve quality and cost of healthcare delivered to patients.

- Understanding the future role for biopharmaceuticals in human health, market dynamics, and implications for manufacturers, public and private payers, providers, patients, pharmacists and distributors.
- Researching the role of technology in health system products, processes and delivery systems and the business and policy systems that drive innovation.

\section{GUIDING PRINCIPLES}

The Institute operates from a set of Guiding Principles:

- Healthcare solutions of the future require fact based scientific evidence, expert analysis of information, technology, ingenuity and a focus on individuals.

- Rigorous analysis must be applied to vast amounts of timely, high-quality and relevant data to provide value and move healthcare forward.

- Collaboration across all stakeholders in the public and private sectors is critical to advancing healthcare solutions.

- Insights gained from information and analysis should be made widely available to healthcare stakeholders.

- Protecting individual privacy is essential, so research will be based on the use of non-identified patient information and provider information will be aggregated.

- Information will be used responsibly to advance research, inform discourse, achieve better healthcare and improve the health of all people. 
CONTACT US

info@iqviainstitute.org iqviainstitute.org 